\title{
AN ALGEBRAIC CLASSIFICATION OF CERTAIN SIMPLE EVEN-DIMENSIONAL KNOTS
}

\author{
BY \\ C. KEARTON ${ }^{1}$
}

\begin{abstract}
The simple $2 q$-knots, $q \geqslant 4$, for which $H_{q}(\tilde{K})$ contains no Z-torsion, are classified by means of Hermitian duality pairings on their homology and homotopy modules.
\end{abstract}

0. Introduction. An $n$-knot $k$ is a locally flat pair $\left(S^{n+2}, S^{n}\right)$ in the piecewise-linear category, where $S^{n}$ denotes the $n$-dimensional sphere. The exterior $K$ of $k$ is the closure of the complement of a regular neighbourhood of $S^{n}$ in $S^{n+2}$, and $k$ is simple if $K$ has the homotopy [ $(n-1) / 2]$-type of a circle. By Alexander-Poincare duality, $K$ has the homology of a circle, and so the kernel of the Hurewicz map $\pi_{1}(K) \rightarrow$ $H_{1}(K)=(t:)$ corresponds to a covering space $\tilde{K}$ of $K$ which has the infinite cyclic group $(t:)$ as its group of covering transformations. In the case of a simple knot $(n>1), \tilde{K}$ is the universal cover of $K$. The homology groups $H_{*}(\tilde{K})$ are modules over $\Lambda=\mathbf{Z}\left[t, t^{-1}\right]$, and are finitely generated because $K$ is a finite complex. They are also $\Lambda$-torsion modules.

The simple $(2 q-1)$-knots have just one nonzero module $H_{q}(\tilde{K})$, and there is a nonsingular $(-1)^{q-1}$-Hermitian pairing [ , ]: $H_{q}(\tilde{K}) \times H_{q}(\tilde{K}) \rightarrow \Lambda_{0} / \Lambda$, known as the Blanchfield pairing. Here $\Lambda_{0}$ denotes the field of fractions of $\Lambda$, and conjugation is the linear extension of $t \mapsto t^{-1}$. For $q>1$, the simple $(2 q-1)$-knots are classified by the pair $\left(H_{q}(\tilde{K}),[],\right)$; see [K5]. There is an alternative classification of these knots, due to Levine, in terms of their Seifert matrices modulo $S$-equivalence; see [L3].

The simple $2 q$-knots have proved more difficult to classify. There are two nonzero homology modules, $H_{q}(\tilde{K})$ and $H_{q+1}(\tilde{K})$. In the case when $H_{q}(\tilde{K})$ is a Z-torsionmodule, we have $H_{q+1}(\tilde{K})=0$ and the knots are classified by the Levine pairing $H_{q}(\tilde{K}) \times H_{q}(\tilde{K}) \rightarrow Q / \mathbf{Z}$ together with an isometry (see [Ko2] for details), provided $q \geqslant 4$. This result requires also that $H_{q}(\tilde{K})$ has no 2 -torsion. Some progress analogous to Levine's results on the odd-dimensional case has also been made. In [K6], the simple $2 q$-knots, $q \geqslant 4$, are classified in terms of certain pairings over $\mathbf{Z}$ and $\mathbf{Z}_{2}$, modulo an equivalence relation, provided that $H_{q}(\tilde{K})$ has no 2-torsion. Such

Received by the editors February 4, 1981 and, in revised form, July 24, 1981.

1980 Mathematics Subject Classification. Primary 57Q45.

Key words and phrases. High dimensional knot, homotopy pairing.

${ }^{1}$ This paper was written while the author was in receipt of a Research Grant from the Science Research Council of Great Britain. 
knots are called odd simple $2 q$-knots. Kojima [Ko] has classified fibred odd simple $2 q$-knots, $q \geqslant 4$, where $H_{q}(\tilde{K})$ is $\mathbf{Z}$-torsion-free, in terms of Seifert matrices over $\mathbf{Z}$ and $\mathbf{Z}_{2}$, modulo an equivalence relation.

M. S. Faber [Fa] has classified the $n$-knots bounding $r$-connected Seifert surfaces, where $6 \leqslant n+1 \leqslant 3 r$, in terms of stable homotopy theory and Spanier-Whitehead duality. As in [L3], this involves an equivalence relation induced by ambient surgery on the Seifert surface.

In this paper we obtain results in the spirit of [K5] and [Ko2], by classifying the simple $2 q$-knots, $q \geqslant 4$, for which $H_{q}(\tilde{K})$ is Z-torsion-free, in terms of the homology and homotopy modules of $\tilde{K}$, together with certain Hermitian pairings obtained from Blanchfield duality and an analogous version of homotopy linking. The plan of the paper is as follows.

In $\$ 1$ the invariants used to classify the knots are described and their properties proved. We define $\mathcal{H}_{i}(\tilde{K})=H_{i}(\tilde{K}) / 2 H_{i}(\tilde{K}), \Pi_{q+1}(\tilde{K})=\pi_{q+1}(\tilde{K}) / 2 \pi_{q+1}(\tilde{K})$, and obtain a short exact sequence

$$
\mathcal{E}(\tilde{K}): \mathcal{H}_{q}(\tilde{K}) \stackrel{\Omega}{\mapsto} \Pi_{q+1}(\tilde{K}) \stackrel{H}{\rightarrow} \mathcal{H}_{q+1}(\tilde{K})
$$

of $\Gamma$-modules, where $\Gamma=\mathbf{Z}_{2}\left[t, t^{-1}\right]$. The map $H$ is induced by the Hurewicz map $\pi_{q+1}(\tilde{K}) \rightarrow H_{q+1}(\tilde{K})$, and the map $\Omega$ is induced by the composite of the inverse of the Hurewicz isomorphism $\pi_{q}(\tilde{K}) \rightarrow H_{q}(\tilde{K})$ with the map $\pi_{q}(K) \rightarrow \pi_{q+1}(\tilde{K})$ obtained by composition with the nonzero element of $\pi_{q+1}\left(S^{q}\right)$. There are nonsingular Hermitian pairings

$$
\begin{gathered}
{[,]_{\tilde{K}}: \Pi_{q+1}(\tilde{K}) \times \Pi_{q+1}(\tilde{K}) \rightarrow \Gamma_{0} / \Gamma,} \\
{ }^{\theta}\langle,\rangle_{\tilde{K}}: \mathcal{H}_{q+1}(\tilde{K}) \times \mathcal{H}_{q}(\tilde{K}) \rightarrow \Gamma_{0} / \Gamma
\end{gathered}
$$

where $\Gamma_{0}$ is the field of fractions of $\Gamma$, related by

$$
[u, \Omega(v)]={ }^{\theta}\langle H(u), v\rangle \text { for all } u \in \pi_{q+1}(\tilde{K}), v \in \mathcal{H}_{q}(\tilde{K}) .
$$

The quotient map $H_{q}(\tilde{K}) \rightarrow \mathscr{H}_{q}(\tilde{K})$ is denoted by $p_{q}(\tilde{K})$. The quintuple $\left(\mathcal{E}(\tilde{K}), H_{q}(\tilde{K}), p_{q}(\tilde{K}),[,]_{\tilde{K}},{ }^{\theta}\langle,\rangle_{\tilde{K}}\right)$ is called an $F$-form.

In $\S 2$ the main results of the paper are stated. These amount to the assertion that there is a bijection between the set of simple $\mathbf{Z}$-torsion-free $2 q$-knots, $q \geqslant 6$, and the set of $F$-forms.

In order to show that isometric $F$-forms come from isotopic knots, the first step is to reconstruct from an $F$-form $\left((\tilde{K}), H_{q}(\tilde{K}), p_{q}(\tilde{K}),\left[,{ }_{\tilde{K}},{ }^{\theta}\langle,\rangle_{\tilde{K}}\right)\right.$ the $\Lambda$-modules $H_{q}(\tilde{K}), H_{q+1}(\tilde{K}), \pi_{q+1}(\tilde{K})$, the Hurewicz and other maps, and the Blanchfield and homotopy pairings between them. This is accomplished in $\S 3$.

A presentation of an $n$-knot is an embedding of $S^{n}$ in $S^{n+1} \times I$ together with a collared handle decomposition of $S^{n}$ such that each handle is embedded in a level $S^{n+1}$ and each collar is embedded productwise along the $I$ direction. Each $r$-handle of $S^{n}$ is associated with an $(r+1)$-handle of $K$. A simple $2 q$-knot, $q \geqslant 4$, has a presentation with one 0 -handle, some $q-1, q$, and $(q+1)$-handles, and a $2 q$ handle of $S^{2 q}$. Via the associated handle decomposition of $K$, we obtain matrices 
over $\Lambda$ and $\Gamma$ which present the $F$-form of the knot. That is to say, we obtain a $\Lambda$-matrix which presents $H_{q}(\tilde{K})$, an Hermitian $\Gamma_{0}$-matrix representing [ , ] ${ }_{K}$, and so on. The strategy of the proof is to show that two sets of matrices presenting isometric $F$-forms are equivalent in the sense that one set can be transformed into the other by a sequence of elementary matrix moves (such as adding one row to another). Then we show that any such matrix move can be realised geometrically by a handle move (such as isotoping one handle over another). Finally we show that if two knots give rise to the same set of matrices, then they are isotopic, by invoking theorems of the "homotopy implies isotopy" type.

The $q, q+1$, and $(q+2)$-handles of $K$ give rise to a sequence of free $\Lambda$-modules, $C_{q+2}(\tilde{K}) \rightarrow C_{q+1}(\tilde{K}) \rightarrow C_{q}(\tilde{K})$, which gives rise to the homology modules $H_{q}(\tilde{K})$, $H_{q+1}(\tilde{K})$ in the usual way. In $\S 4$ we show, in a purely algebraic setting, that we can split this sequence up into two presentations $\Lambda^{m} \rightarrow \Lambda^{n} \rightarrow H_{i}(\tilde{K})$ for $i=q, q+1$.

The $(q-1)$-handles of $S^{2 q}$ are unknotted in their level, in the sense that they are ambient isotopic to a standard embedding. We are then faced with the problem of isotoping the $q$-handles about in their level; unfortunately the classical "homotopy implies isotopy" theorems are not sufficient for the purpose because the ambient manifold is not simply-connected, so $\$ 5$ is devoted to proving the isotopy results that will be needed later in the paper.

Because of the algebraic results proved in $\S 4$, it is possible to obtain for the knot a presentation with one 0-handle, $m(q-1)$ and $n q$-handles, $m q$ and $n(q+1)$ handles, and a $2 q$-handle, and moreover we may assume that the $m(q-1)$ and $n$ $q$-handles are unknotted. Thus $\S 6$ is devoted to a description of $K \cap$ (a level between the $m(q-1)$ and $n q$-handles, and the $m q$-handles and $n(q+1)$-handles), and of its homology and homotopy modules. The embeddings of the latter set of handles determine elements of these modules, and hence we obtain the matrices which present the $F$-form of the knot. In $\$ 7$ we justify some of the assertions above about handles being unknotted.

In $\S 8$ we look more closely at the relationship between the handles of $S^{2 q}$ and the handles of $\tilde{K}$, and justify the assertions made about handle moves inducing matric moves, and the relationships with the presentation of the $F$-form.

In $\$ \S 9$ and 10 we prove the main theorems of the paper. In $\$ 11$ we consider a $(q-1)$-connected Seifert surface of the knot, and show how the $F$-form can be presented in terms of matrices obtained from the surface in the usual way by means of linking numbers and "homotopy linking". This technique enables us to extend the range of dimensions for which the main theorems are valid to $q \geqslant 4$.

We conclude with $\$ 12$ in which an example is given of two knots which have isomorphic modules but are distinguished by their Hermitian pairings.

1. The invariants. Let $\Lambda=\mathbf{Z}\left[t, t^{-1}\right]$ and $S=\{f(t) \in \Lambda: f(1)= \pm 1\}$. Let $\Lambda_{S}$ denote $\Lambda$ localised at $S$, so that $\Lambda_{S}=\{f / g: f \in \Lambda, g \in S\}$, and consider $\Lambda_{S} / \Lambda$ as a $\Lambda$-module. If $\mathbf{Z}_{2}$ denotes the field with two elements, set $\Gamma=\mathbf{Z}_{2}\left[t, t^{-1}\right]$; thus $\Gamma$ may be regarded as $\Lambda$ with coefficients reduced $\bmod 2$. Let $\Gamma_{0}$ denote the field of fractions of $\Gamma$. 
The map $\Theta: \Lambda \rightarrow \Gamma$ obtained by reducing coefficients mod 2 extends to a ring homomorphism $\Lambda_{S} \rightarrow \Gamma_{0}$, and the composition $\Lambda_{S} \rightarrow \Gamma_{0} \rightarrow \Gamma_{0} / \Gamma$ of $\Lambda$-module homomorphisms contains $\Lambda$ in its kernel. Thus there is a well-defined $\Lambda$-module homomorphism $\theta: \Lambda_{S} / \Lambda \rightarrow \Gamma_{0} / \Gamma$, with $\operatorname{ker} \theta=2 \Lambda_{S} / \Lambda$.

Conjugation in $\Lambda$ and $\Gamma$ is the linear extension of $t \mapsto t^{-1}$, and is denoted by ${ }^{-}$. This induces a conjugation on $\Lambda_{S} / \Lambda$ and on $\Gamma_{0} / \Gamma$, also denoted by ${ }^{-}$.

If $M$ and $N$ are $\Lambda$-modules, then a pairing

$$
\langle,\rangle: M \times N \rightarrow \Lambda_{S} / \Lambda
$$

is Hermitian if it is linear in the first variable and conjugate linear in the second. If $M=N$, then we also require $\langle,\rangle^{*}=\langle$,$\rangle , where in general$

$$
\langle,\rangle^{*}: N \times M \rightarrow \Lambda_{S} / \Lambda
$$

is defined by $\langle n, m\rangle^{*}=\overline{\langle m, n\rangle}$.

The Hermitian pairing $\langle$,$\rangle defines an adjoint map$

$$
\alpha: M \rightarrow \overline{\operatorname{Hom}}\left(N, \Lambda_{S} / \Lambda\right)
$$

by $\alpha(m)(n)=\langle m, n\rangle$. We say that the pairing $\langle$,$\rangle is nonsingular if the adjoint$ maps of $\langle$,$\rangle and of \langle,\rangle^{*}$ are isomorphisms.

Similar definitions hold with $\Gamma$ in place of $\Lambda$ and $\Gamma_{0}$ in place of $\Lambda_{S}$.

Consider now a simple Z-torsion-free $2 q$-knot with exterior $K$. Thus $H_{*}(\tilde{K})$ is zero except in dimensions $q, q+1$, where it is $\mathbf{Z}$-torsion-free. By Blanchfield duality [B] there is a nonsingular Hermitian pairing of $\Lambda$-modules

$$
H_{q+1}(\tilde{K}, \partial \tilde{K}) \times H_{q}(\tilde{K}) \rightarrow \Lambda_{0} / \Lambda
$$

where $\Lambda_{0}$ is the field of fractions of $\Lambda$. Since there is a canonical isomorphism $H_{q+1}(\tilde{K}) \cong H_{q+1}(\tilde{K}, \partial \tilde{K})$, and the Alexander polynomials satisfy $\Delta_{q+1}(1)= \pm 1=$ $\Delta_{q}(1)$, this induces a nonsingular Hermitian pairing

$$
\langle,\rangle: H_{q+1}(\tilde{K}) \times H_{q}(\tilde{K}) \rightarrow \Lambda_{S} / \Lambda .
$$

Because $\tilde{K}$ is simply-connected we do not need a base-point in discussing the higher homotopy groups $\pi_{q}(\tilde{K})$ and $\pi_{q+1}(\tilde{K})$; see [Hu, Chapter IV] for details.

Let $h_{*}: \pi_{*}(\tilde{K}) \rightarrow H_{*}(\tilde{K})$ denote the Hurewicz homomorphism, and let $\zeta$ denote the nonzero element of $\pi_{q+1}\left(S^{q}\right) \cong \mathbf{Z}_{2}(q \geqslant 3)$. Define the map $s: \pi_{q}(\tilde{K}) \rightarrow \pi_{q+1}(\tilde{K})$ by $s(x)=x \xi$; clearly $s$ is a homomorphism of $\Lambda$-modules. By Hurewicz's theorem, $h_{q}$ is an isomorphism, so we can define $\omega=s h_{q}^{-1}$.

LEMMA 1.1. Provided $q \geqslant 3$, the sequence

$$
H_{q}(\tilde{K}) \stackrel{\omega}{\rightarrow} \pi_{q+1}(\tilde{K}) \stackrel{h_{q+1}}{\rightarrow} H_{q+1}(\tilde{K}) \rightarrow 0
$$

is exact.

Proof. By a result of G. W. Whitehead, $h_{q+1}$ is onto since $q \geqslant 2$ [Hu, p. 167]. 
Let $K^{q}$ denote the $q$-skeleton of $K$ in some triangulation and consider the commutative ladder:

$$
\begin{array}{ccccc}
\pi_{q+1}\left(\tilde{K}^{q}\right) & \stackrel{j}{\rightarrow} & \pi_{q+1}(\tilde{K}) & \stackrel{J}{\rightarrow} & \pi_{q+1}\left(\tilde{K}, \tilde{K}^{q}\right) \\
\downarrow & & \downarrow h_{q+1} & & \downarrow h_{q+1}^{\prime} \\
H_{q+1}\left(\tilde{K}^{q}\right) & \stackrel{j_{*}}{\rightarrow} & H_{q+1}(\tilde{K}) & \stackrel{J_{*}}{\rightarrow} & H_{q+1}\left(\tilde{K}, \tilde{K}^{q}\right)
\end{array}
$$

The map $h_{q+1}^{\prime}$ is an isomorphism by the Hurewicz theorem, since $\left(\tilde{K}, \tilde{K}^{q}\right)$ is $q$-connected and $\tilde{K}^{q}$ is simply-connected. Since $H_{q+1}\left(\tilde{K}^{q}\right)=0, h_{q+1} j=0$, and since $\operatorname{Im} j=\operatorname{Im} s$ we have $h_{q+1} s=0$ and hence $h_{q+1} \omega=0$.

Suppose $x \in \operatorname{ker} h_{q+1}$. Then $h_{q+1}^{\prime} J(x)=J_{*} h_{q+1}(x)=0$, whence $J(x)=0$ as $h_{q+1}^{\prime}$ is an isomorphism. Thus $x \in \operatorname{ker} J=\operatorname{Im} j=\operatorname{Im} s$. So $\operatorname{ker} h_{q+1} \subset \operatorname{Im} s=\operatorname{Im} \omega$.

COROLlaRY 1.2. Im $\omega=\operatorname{ker} h_{q+1}=\mathbf{Z}$-torsion submodule of $\pi_{q+1}(\tilde{K})$.

Proof. Since $H_{q+1}(\tilde{K})$ is Z-torsion-free, the Z-torsion submodule of $\pi_{q+1}(\tilde{K}) \subset$ $\operatorname{ker} h_{q+1}=\operatorname{Im} \omega \subset 2$-torsion submodule of $\pi_{q+1}(\tilde{K})$.

COROllary 1.3. If $\Delta$ is the Alexander polynomial of the knot in dimension $q$, then $\Delta \bar{\Delta}$ annihilates $\pi_{q+1}(\tilde{K})$.

Proof. The Alexander polynomial in dimension $q+1$ is $\bar{\Delta}$, by Blanchfield duality. Thus $\Delta$ annihilates $H_{q}(\tilde{K}), \bar{\Delta}$ annihilates $H_{q+1}(\tilde{K})$, and the result follows at once.

We next define a pairing

$$
\{,\}: \pi_{q+1}(\tilde{K}) \times \pi_{q+1}(\tilde{K}) \rightarrow \Gamma_{0} / \Gamma
$$

where $q \geqslant 3$. Let $u, v \in \pi_{q+1}(\tilde{K})$; by Irwin's theorem, there exist embeddings $x, y$ : $S^{q+1} \rightarrow \tilde{K}$ representing $u, v$ respectively. The Blanchfield intersection pairing

$$
S: H_{q+1}(\tilde{K}) \times H_{q+1}(\tilde{K}) \rightarrow \Lambda
$$

is zero [B], because $H_{q+1}(\tilde{K})$ is a $\Lambda$-torsion module, and so we may isotop $x$ so that $x$ and $t^{r} y$ are disjoint for every integer $r$, where $\left(t^{r} y\right)(z)=t^{r}(y(z))$ for $z \in S^{q+1}$. This is essentially an application of the Whitney Lemma (compare [K1, Theorem 2.1]), and uses $q \geqslant 3$.

Suppose that $\alpha$ is an element of $\Lambda$ such that $\Theta(\alpha) \neq 0$ and $\alpha x=\partial c$, where $c$ : $B^{q+2} \rightarrow \tilde{K}$. Such an $\alpha$ exists by Corollary 1.3. As in [K1], we may arrange that for each integer $r$, there are sets $X_{r}, Y_{r}, Z_{r}$ satisfying:

(i) the $X_{r}$ are disjoint subsets of int $B^{q+2}$, finitely many of which are $(q+2)$-balls and the rest are empty;

(ii) the $Y_{r}$ are disjoint subsets of $S^{q+1}$, finitely many of which are $(q+1)$-balls and the rest are empty;

(iii) the $Z_{r}$ are disjoint subsets of $\tilde{K}$, finitely many of which are $(2 q+2)$-balls and the rest are empty;

(iv) $X_{r}=c^{-1} Z_{r}, Y_{r}=\left(t^{r} y\right)^{-1} Z_{r}$; 
(v) $\operatorname{Im} c \cap \operatorname{Im} t^{r} y \subset$ int $Z_{r}$;

(vi) $S(c) \cap X_{r}=\varnothing$.

This too requires $q \geqslant 3$.

The map

$$
c \mid \partial X_{r}: \partial X_{r} \rightarrow \partial Z_{r}-t^{r} y\left(\partial Y_{r}\right) \simeq S^{q}
$$

gives an element $I\left(c, t^{r} y\right) \in \pi^{1} \cong \pi_{q+1}\left(S^{q}\right) \cong \mathbf{Z}_{2}$, and we define

$$
T(c, y)=\sum_{-\infty<r<\infty} I\left(c, t^{r} y\right) t^{r} \in \Gamma \text {. }
$$

LEMMA 1.4. $T(c, y)$ is independent of the choice of $c$.

Proof. Let $c^{\prime}: B^{q+2} \rightarrow \tilde{K}$ be another map with $\partial c^{\prime}=\alpha x$. Then $T\left(c-c^{\prime}, y\right)=$ $T(c, y)-T\left(c^{\prime}, y\right)$, and $c-c^{\prime}$ represents an element of $\pi_{q+2}(\tilde{K})$. By Corollary 1.3, there exists $\beta \in \Lambda$ such that $\Theta(\beta) \neq 0$ and $\bar{\beta} v=0$. Thus $0=T\left(c-c^{\prime}, \bar{\beta} y\right)=$ $\Theta(\beta) \cdot T\left(c-c^{\prime}, y\right)$, and so $T\left(c-c^{\prime}, y\right)=0$.

Suppose that $x^{\prime}$ is another representative of $u$. Then $x-x^{\prime}=\partial a$, for some $a$ : $S^{q+1} \times I \rightarrow \tilde{K}$. If we take $c^{\prime}: B^{q+2} \rightarrow \tilde{K}$ as the union of $c$ and $\alpha a$, so that $\alpha x^{\prime}=\partial c^{\prime}$, then $T\left(c^{\prime}, y\right)=T(c+\alpha a, y)=T(c, y)+\Theta(\alpha) T(a, y)$. Defining

$$
\{u, v\}=\frac{1}{\Theta(\alpha)} \cdot T(c, y) \in \Gamma_{0} / \Gamma
$$

we see that $\{u, v\}$ is independent of the choice of $c$ and $x$.

Suppose now that $\beta \in \Lambda$ is such that $\Theta(\beta) \neq 0$ and $\bar{\beta} v=0$. Then $\bar{\beta} y=\partial d$ for some $d: B^{q+2} \rightarrow \tilde{K}$, and we can form $T(x, d)$. As in [K1], $T(x, d)=\overline{T(d, x)}$, and so is independent of the choice of $y$ and $d$.

LeMMA 1.5. Let $c, d: B^{q+2} \rightarrow$ int $\tilde{K}$ be maps with $\partial c=c\left|\partial B^{q+2}, \partial d=d\right| \partial B^{q+2}$ such that $\operatorname{Im} \partial c \cap \operatorname{Im} \partial d=\varnothing$. Then $I(\partial c, d)=I(c, \partial d)$, provided $q>4$.

Proof. The dimension of $\operatorname{Im}(\partial d)$ is at most $q+1$, and so $\tilde{K}-\operatorname{Im}(\partial d)$ is $(q-1)$-connected. Thus by Irwin's Theorem we may homotop $\partial c$ in $\tilde{K}-\operatorname{Im}(\partial d)$ to be an embedding, and this extends to a homotopy of $c$. The same procedure then applies with $c$ and $d$ interchanged, and so we may assume that $\partial c, \partial d$ are disjoint embeddings. By general position we may assume that $\operatorname{dim}(\operatorname{Im} \partial c \cap \operatorname{Im} d) \leqslant 1$, $\operatorname{dim}(\operatorname{Im} c \cap \operatorname{Im} \partial d) \leqslant 1$, and that the map $c \cup d: B^{q+2} \cup B^{q+2} \rightarrow \tilde{K}$ has no triple points. In particular, the last requirement implies that the self-intersections of $\operatorname{Im} c$ do not meet Im $d$, and vice-versa.

By engulfing, there exist collapsible sets $C_{1}, D_{1} \subset S^{q+1}=\partial B^{q+2}$ such that

$$
\begin{array}{ll}
(\partial c)^{-1}(\operatorname{Im} \partial c \cap \operatorname{Im} d) \subset C_{1}, & \operatorname{dim} C_{1} \leqslant 2, \\
(\partial d)^{-1}(\operatorname{Im} c \cap \operatorname{Im} \partial d) \subset D_{1}, & \operatorname{dim} D_{1} \leqslant 2 .
\end{array}
$$

Let $S(f)$ denote the singular set of the map $f$. By general position we can arrange that $\operatorname{dim} S(c \cup d) \leqslant 2$, and so by engulfing there exist $C, D \subset B^{q+2}$ such that

$$
\begin{array}{ll}
S(c) \cup c^{-1}(\operatorname{Im} c \cap \operatorname{Im} d) \subset C \searrow C_{1}=C \cap S^{q+1}, & \operatorname{dim} C \leqslant 3, \\
S(d) \cup d^{-1}(\operatorname{Im} c \cap \operatorname{Im} d) \subset D \searrow D_{1}=D \cap S^{q+1}, & \operatorname{dim} D \leqslant 3 .
\end{array}
$$


Furthermore, there exists a collapsible $E \subset$ Int $\tilde{K}$ such that $c(C) \cup d(D) \subset E$, $\operatorname{dim} E \leqslant 4$,

$$
\left.\begin{array}{l}
\operatorname{dim}\left(E \cap c\left(B^{q+2}-C\right)\right) \\
\operatorname{dim}\left(E \cap d\left(B^{q+2}-D\right)\right)
\end{array}\right\} \leqslant 4+q+2-(2 q+2)=4-q<0 .
$$

Since $C$ is collapsible and $\operatorname{dim} B^{q+2}-\operatorname{dim} C \geqslant q-1>3, B^{q+2} \searrow C$; and as $S(c) \subset C$, this means that $\operatorname{Im} c \searrow c(C)$. Similarly $\operatorname{Im} d \searrow d(D)$. Since $\operatorname{Im} c \cap \operatorname{Im} d \subset$ $c(C) \cap c(D)$, we see that

$$
\operatorname{Im} c \cup \operatorname{Im} d \cup E \searrow c(C) \cup c(D) \cup E=E \searrow \text { point. }
$$

Thus a regular neighbourhood of $\operatorname{Im} c \cup \operatorname{Im} d \cup E$ is a ball, $B^{2 q+2} . I(\partial c, d)$ is given by an element of $\pi_{q}\left(S^{q-1}\right)$ which under the suspension homomorphism goes to the linking element of $\partial c$ and $\partial d$ in $B^{2 q+2}, L(\partial c, \partial d) \in \pi_{q+1}\left(S^{q}\right)$. A similar statement is true of $I(c, \partial d)$, and so the two terms are equal as members of $\pi^{1}$.

Corollary 1.6. $T(\partial c, d)=T(c, \partial d)$.

Returning to the definition of $\{u, v\}$, we have

$$
\begin{aligned}
\{u, v\} & =\frac{1}{\Theta(\alpha)} T(c, y)=\frac{1}{\Theta(\alpha \beta)} \cdot T(c, \bar{\beta} y)=\frac{1}{\Theta(\alpha \beta)} \cdot T(c, \partial d) \\
& =\frac{1}{\Theta(\alpha \beta)} \cdot T(\partial c, d)=\frac{1}{\Theta(\alpha \beta)} \cdot T(\alpha x, d)=\frac{1}{\Theta(\beta)} \cdot T(x, d) .
\end{aligned}
$$

This shows that $\{u, v\}$ is independent of the choice of $x$ and of the choice of $y$, and of $\alpha$ and $\beta$, and of $c$ and $d$; and hence is well defined.

Proposition 1.7. $\{$,$\} is Hermitian. That is,$

$$
\begin{aligned}
\{\alpha u+\beta v, w\} & =\Theta(\alpha)\{u, w\}+\Theta(\beta)\{v, w\}, \\
\{u, \alpha v+\beta w\} & =\Theta(\bar{\alpha})\{u, v\}+\Theta(\bar{\beta})\{u, w\}, \\
\{u, v\} & =\overline{\{v, u\}}
\end{aligned}
$$

for all $u, v, w \in \pi_{q+1}(\tilde{K})$ and all $\alpha, \beta \in \Lambda$.

Proof. The first two assertions follow easily from the definition (compare [K1, Proposition 3.1]). The last assertion is proved by

$$
\{u, v\}=\frac{1}{\Theta(\alpha)} \cdot T(c, y)=\frac{1}{\Theta(\alpha)} \cdot \overline{T(y, c)}=\overline{\frac{1}{\Theta(\bar{\alpha})} \cdot T(y, c)}=\overline{\{v, u\}} \text {. }
$$

LEMMA 1.8. For all $u \in \pi_{q+1}(\tilde{K})$ and all $v \in H_{q}(\tilde{K})$, we have

$$
\{u, \omega(v)\}=\theta\left\langle h_{q+1}(u), v\right\rangle \text {. }
$$

Proof. Let $x: S^{q+1} \hookrightarrow \tilde{K}$ represent $u$, and let $c: B^{q+2} \rightarrow \tilde{K}$ be such that $\partial c=\alpha x$, for some $\alpha \in \Lambda$ with $\Theta(\alpha) \neq 0$. Let $y: S^{q+1} \hookrightarrow \tilde{K}$ represent $v$, with $\operatorname{Im} x \cap \operatorname{Im} y=\varnothing$. We may assume that $\operatorname{Im} c \cap \operatorname{Im} y$ consists of isolated points. Since $\omega(v)$ is represented by $y \zeta$, the result follows from the construction in [K1, Proposition 3.2]. 
COROllary 1.9. $\{$,$\} vanishes on \operatorname{Im} \omega \times \operatorname{Im} \omega$.

Proof. $\operatorname{Im} \omega=\operatorname{ker} h_{q+1}$.

LEMMA 1.10. $\operatorname{ker} \omega=2 H_{q}(\tilde{K})$.

Proof. If $x \in 2 \pi_{q}(\tilde{K})$, then $x=2 y$ and $s(x)=s(2 y)=2 s(y)=2 y \zeta=0$. Thus $\operatorname{ker} \omega \supset 2 H_{q}(\tilde{K})$.

Conversely, suppose that $v \in \operatorname{ker} \omega$. Then

$$
\theta\left\langle h_{q+1}(u), v\right\rangle=\{u, \omega(v)\}=0, \quad \text { for all } u \in \pi_{q+1}(K) .
$$

Thus $\left\langle h_{q+1}(u), v\right\rangle \in 2 \Lambda_{S} / \Lambda$ for all $u \in \pi_{q+1}(\tilde{K})$. Since $h_{q+1}$ is an epimorphism, and $\langle$,$\rangle is nonsingular, this implies that v \in 2 H_{q}(\tilde{K})$, and so $\operatorname{ker} \omega \subset 2 H_{q}(\tilde{K})$.

Now we define $\Pi_{q+1}(\tilde{K})=\pi_{q+1}(\tilde{K}) / 2 \pi_{q+1}(\tilde{K})$, with quotient map $\eta$, so that $\Pi_{q+1}(\tilde{K})$ is a $\Gamma$-torsion module. Moreover, $\{$,$\} induces a pairing$

$$
[,]: \Pi_{q+1}(\tilde{K}) \times \Pi_{q+1}(\tilde{K}) \rightarrow \Gamma_{0} / \Gamma,
$$

since $\{$,$\} vanishes on 2 \pi_{q+1}(\tilde{K}) \times \pi_{q+1}(\tilde{K})$.

Define $\mathcal{H}_{i}(\tilde{K})=H_{i}(\tilde{K}) / 2 H_{i}(\tilde{K})$ for $i=q, q+1$, and let $p_{i}$ denote the quotient map in each case. We define a map $\Omega: \mathcal{H}_{q}(\tilde{K}) \rightarrow \Pi_{q+1}(\tilde{K})$ as follows. Given $v \in \mathcal{H}_{q}(\tilde{K})$, there exists $v_{1} \in H_{q}(\tilde{K})$ with $p_{q}\left(v_{1}\right)=v$. Let $\Omega(v)=\eta \omega\left(v_{1}\right)$. By Lemma $1.10, \Omega$ is well defined.

Next we define a map $H: \Pi_{q+1}(\tilde{K}) \rightarrow \mathcal{H}_{q+1}(\tilde{K})$. Given $v \in \Pi_{q+1}(\tilde{K})$, there exists $u_{1} \in \pi_{q+1}(\tilde{K})$ with $\eta\left(u_{1}\right)=u$. Define $H(u)=p_{q+1} h_{q+1}\left(u_{1}\right)$. This is well defined because $h_{q+1}\left(2 \pi_{q+1}(\tilde{K})\right) \subset 2 H_{q+1}(\tilde{K})=\operatorname{ker} p_{q+1}$. Clearly $H$ is an epimorphism.

LEMMA 1.11. $\operatorname{ker} H=\operatorname{Im} \Omega$.

Proof. $H(u)=0 \Rightarrow p_{q+1} h_{q+1}\left(u_{1}\right)=0$ where $u_{1} \in \pi_{q+1}(\tilde{K})$ and $\eta\left(u_{1}\right)=u$.

$$
\begin{aligned}
p_{q+1} h_{q+1}\left(u_{1}\right) & =0 \Rightarrow h_{q+1}\left(u_{1}\right) \in 2 H_{q+1}(\tilde{K}) \\
& \Rightarrow h_{q+1}\left(u_{1}\right)=2 h_{q+1}\left(v_{1}\right), \quad \text { some } v_{1} \in \pi_{q+1}(\tilde{K}) \\
& \Rightarrow u_{1}-2 v_{1} \in \operatorname{Im} \omega \quad(\text { Lemma 1.1) } \\
& \Rightarrow u_{1}-2 v_{1}=\omega(w) \\
& \Rightarrow u=\eta\left(u_{1}\right)=\eta \omega(w)=\Omega(w) .
\end{aligned}
$$

Therefore ker $H \subset \operatorname{Im} \Omega$. Conversely,

$$
\begin{aligned}
u & =\Omega(w) \Rightarrow u=\eta \omega\left(w_{1}\right), \quad \text { where } p_{q}\left(w_{1}\right)=w, \\
& \Rightarrow H(u)=p_{q+1} h_{q+1} \omega\left(w_{1}\right)=0 \quad(\text { Lemma 1.1). }
\end{aligned}
$$

Therefore $\operatorname{Im} \Omega \subset \operatorname{ker} H$.

For $x \in \mathcal{K}_{q+1}(\tilde{K}), y \in \mathcal{H}_{q}(\tilde{K})$, define

$$
{ }^{\theta}\langle x, y\rangle=\theta(\langle u, v\rangle)
$$

where $p_{q+1}(u)=x, p_{q}(v)=y$. Clearly this pairing is well defined. 
Proposition 1.12. For all $u \in \Pi_{q+1}(\tilde{K})$ and all $v \in \mathcal{H}_{q}(\tilde{K})$,

$$
[u, \Omega(v)]={ }^{\theta}\langle H(u), v\rangle .
$$

Proof. Follows easily from Lemma 1.8 .

L"MMA $1.13 .{ }^{\theta}\langle$,$\rangle is nonsingular.$

Proof. Let $x \in \mathcal{H}_{q+1}(\tilde{K})$, and $p_{q+1}(u)=x$. Then

$$
\begin{aligned}
\theta_{\langle x, y\rangle} & =0 \quad \text { for all } y \in \mathcal{H}_{q}(\tilde{K}) \\
& \Rightarrow \theta(\langle u, v\rangle)=0 \text { for all } v \in H_{q}(\tilde{K}) \\
& \Rightarrow\langle u, v\rangle \in 2 \Lambda_{S} / \Lambda \quad \text { for all } v \in H_{q}(\tilde{K}) \\
& \Rightarrow u \in 2 H_{q}(\tilde{K}) \text { since }\langle,\rangle \text { is nonsingular } \\
& \Rightarrow x=p_{q+1}(u)=0 .
\end{aligned}
$$

Now let $f \in \overline{\operatorname{Hom}}\left(\mathcal{H}_{q}(\tilde{K}), \Gamma_{0} / \Gamma\right)$, and define $f_{1} \in \overline{\operatorname{Hom}}\left(H_{q}(\tilde{K}), \Gamma_{0} / \Gamma\right)$ by $f_{1}(v)=$ $f\left(p_{q}(v)\right)$ for all $v \in H_{q}(\tilde{K})$. The short exact sequence

$$
\operatorname{ker} \theta>\Lambda_{S} / \Lambda \stackrel{\theta}{\rightarrow} \Gamma_{0} / \Gamma
$$

gives rise to an exact sequence

$$
\overline{\operatorname{Hom}}\left(H_{q}(\tilde{K}), \Lambda_{S} / \Lambda\right) \rightarrow \overline{\operatorname{Hom}}\left(H_{q}(\tilde{K}), \Gamma_{0} / \Gamma\right) \rightarrow \overline{\operatorname{Ext}}\left(H_{q}(\tilde{K}), \operatorname{ker} \theta\right) .
$$

As we remarked above, $\operatorname{ker} \theta=2 \Lambda_{S} / \Lambda$, and of course multiplication by 2 yields an isomorphism $\Lambda_{S} / \Lambda \underset{\sim}{\sim} 2 \Lambda_{S} / \Lambda$. By [K2, Lemma 2.3], it follows that $\overline{\operatorname{Ext}}\left(H_{q}(\tilde{K}), \operatorname{ker} \theta\right)=0$, since $H_{q}(\tilde{K})$ has a presentation by a square matrix [Ke, Lemma II.12]. Thus there exists $f_{2} \in \overline{\operatorname{Hom}}\left(H_{q}(\tilde{K}), \Lambda_{s} / \Lambda\right)$ such that $\theta f_{2}=f_{1}$.

Since $\langle$,$\rangle is nonsingular, there exists u \in H_{q+1}(\tilde{K})$ such that $\langle u, v\rangle=f_{2}(v)$ for all $v \in H_{q}(\tilde{K})$. Thus $\theta\langle u, v\rangle=\theta f_{2}(v)=f_{1}(v)=f\left(p_{q}(v)\right)$, and so setting $x=p_{q+1}(u)$, $y=p_{q}(v)$, we have ${ }^{\theta}\langle x, y\rangle=f(y)$ for all $y \in \mathscr{H}_{q}(K)$.

We have thus shown that the adjoint map $\mathcal{H}_{q+1}(\tilde{K}) \rightarrow \overline{\operatorname{Hom}}\left(\mathcal{H}_{q}(\tilde{K}), \Gamma_{0} / \Gamma\right)$ is an isomorphism. The proof that the adjoint of ${ }^{\theta}\langle,\rangle^{*}$ is an isomorphism is similar.

LEMMA 1.14. $\Omega$ is a monomorphism.

Proof. Suppose that $\Omega(u)=0$. Then $\eta \omega\left(u_{1}\right)=0$, where $u_{1} \in H_{q}(\tilde{K})$ and $u=$ $p_{q}\left(u_{1}\right)$. So $\omega\left(u_{1}\right) \in \operatorname{ker} \eta=2 \pi_{q+1}(\tilde{K})$. But $\operatorname{Im} \omega \subset 2$-torsion of $\pi_{q+1}(\tilde{K})=\mathbf{Z}$-torsion of $\pi_{q+1}(\tilde{K})$; see the proof of Corollary 1.2; and so $\operatorname{Im} \omega \cap 2 \pi_{q+1}(\tilde{K})=\{0\}$. Thus $\omega\left(u_{1}\right)=0$, and by Lemma $1.10, u_{1} \in 2 H_{q}(\tilde{K})=\operatorname{ker} p_{q}$, so $u=p_{q}\left(u_{1}\right)=0$.

COROLlary 1.15. The sequence

$$
0 \rightarrow \mathcal{H}_{q}(\tilde{K}) \stackrel{\Omega}{\rightarrow} \Pi_{q+1}(\tilde{K}) \stackrel{H}{\rightarrow} \mathcal{H}_{q+1}(\tilde{K}) \rightarrow 0
$$

is exact.

Proof. Lemmas 1.11 and 1.14. 
Proposition 1.16. The pairing [,] is nonsingular and Hermitian.

Proof. It is Hermitian because $\{$,$\} is Hermitian.$

Suppose that $[u, v]=0$ for all $v \in \Pi_{q+1}(\tilde{K})$. Then by Proposition 1.12, ${ }^{\theta}\langle H(u), x\rangle=0$ for all $x \in \mathcal{H}_{q}(\tilde{K})$, and so by Lemma $1.13 H(u)=0$. Thus by Lemma 1.11,u $=\Omega\left(u^{\prime}\right)$ for some $u^{\prime} \in \mathcal{H}_{q}(\tilde{K})$, and by Proposition 1.12,

$$
{ }^{\theta}\left\langle H(v), u^{\prime}\right\rangle=\left[v, \Omega\left(u^{\prime}\right)\right]=\overline{[u, v]}=0 \text { for all } v \in \Pi_{q+1}(\tilde{K}) .
$$

So by Lemma $1.13, u^{\prime}=0$ since $H$ is an epimorphism, and hence $u=0$. The adjoint of $[$,$] is thus a monomorphism.$

Let $f \in \overline{\operatorname{Hom}}\left(\Pi_{q+1}(\tilde{K}), \Gamma_{0} / \Gamma\right)$. Since ${ }^{\theta}\langle$,$\rangle is nonsingular, there exists u^{\prime} \in$ $\Pi_{q+1}(\tilde{K})$ such that $\left[u^{\prime}, \Omega(y)\right]={ }^{\theta}\left\langle H\left(u^{\prime}\right), y\right\rangle=f(\Omega(y))$ for all $y \in \mathcal{K}_{q}(\tilde{K})$.

Set $g(v)=\left[u^{\prime}, v\right]-f(v)$, so that $g \in \overline{\operatorname{Hom}}\left(\Pi_{q+1}(\tilde{K}), \Gamma_{0} / \Gamma\right)$. Then $g(\Omega(y))=0$ for all $y \in \mathcal{H}_{q}(\tilde{K})$, and so $g$ induces an element $g_{1} \in \overline{\operatorname{Hom}}\left(\mathcal{H}_{q+1}(\tilde{K}), \Gamma_{0} / \Gamma\right)$ such that $g_{1}(H(v))=g(v)$ for all $v \in \Pi_{q+1}(\tilde{K})$. Since ${ }^{\theta}\langle$,$\rangle is nonsingular, there exists$ $x \in \mathcal{H}_{q}(\tilde{K})$ such that

$$
{ }^{\theta}\langle x, y\rangle^{*}=\overline{\theta_{\langle y, x\rangle}}=g_{1}(y)
$$

for all $y \in \mathcal{H}_{q+1}(\tilde{K})$. Then

$$
[\Omega(x), v]=\overline{[v, \Omega(x)]}=\overline{\left.\theta^{\prime} H(v), x\right\rangle}=g_{1}(H(v))=g(v),
$$

and so setting $u=u^{\prime}-\Omega(x)$ we have $[u, v]=f(v)$ for all $v \in \Pi_{q+1}(\tilde{K})$. The adjoint of $[$,$] is therefore an isomorphism.$

2. The main theorems. Before stating the main theorems of the paper, we review some of the results of the previous section. Following Levine [L], we say that a $\Lambda$-module is of type $K$ if it is finitely generated and multiplication by $t-1$ induces an automorphism.

An $F$-form $\left(\mathcal{E}, H_{q}, p_{q},[],,{ }^{\theta}\langle\rangle,\right)$ consists of the following.

(i) A short exact sequence of $\Gamma$-modules

$$
\varepsilon: \mathcal{H}_{q} \stackrel{\Omega}{\longrightarrow} \Pi_{q+1} \stackrel{H}{\rightarrow} \mathcal{H}_{q+1} .
$$

(ii) A nonsingular Hermitian form

$$
[,]: \Pi_{q+1} \times \Pi_{q+1} \rightarrow \Gamma_{0} / \Gamma .
$$

(iii) A nonsingular Hermitian form

$$
{ }^{\theta}\langle,\rangle: \mathcal{H}_{q+1} \times \mathcal{H}_{q} \rightarrow \Gamma_{0} / \Gamma
$$

related to [, ] by

$$
[u, \Omega(v)]={ }^{\theta}\langle H(u), v\rangle \quad \text { for all } u \in \Pi_{q+1}, v \in \mathcal{H}_{q} .
$$

(iv) A Z-torsion-free $\Lambda$-module $H_{q}$ of type $K$.

(v) A short exact sequence of $\Lambda$-modules

$$
2 H_{q} \hookrightarrow H_{q} \stackrel{p_{q}}{\rightarrow} \mathcal{H}_{q},
$$

where $\mathcal{H}_{q}$ is regarded as a $\Lambda$-module via the ring homomorphism $\Theta: \Lambda \rightarrow \Gamma$. 
Two $F$-forms $\left(\mathcal{E}, H_{q}, p_{q},[],,{ }^{\theta}\langle\rangle,\right)$ and $\left(\mathcal{E}^{\prime}, H_{q}^{\prime}, p_{q}^{\prime},[,]^{\prime},{ }^{\theta}\langle,\rangle^{\prime}\right)$ are isometric if there exist isomorphisms $a, \alpha, \beta, \gamma$ such that

$$
\mathcal{E}: \quad \mathcal{H}_{q} \stackrel{\Omega}{\longrightarrow} \Pi_{q+1} \stackrel{H}{\rightarrow} \quad \mathcal{H}_{q+1}
$$

$$
\begin{array}{llllll} 
& \mid \downarrow \chi^{\alpha} & & \mid \downarrow \mathcal{l}^{\beta} & & \mid \downarrow \mathcal{l}^{\gamma} \\
\mathcal{E}^{\prime}: & \mathcal{H}_{q}^{\prime} & \stackrel{\Omega^{\prime}}{\longrightarrow} & \Pi_{q+1}^{\prime} & \stackrel{H^{\prime}}{\rightarrow} & \mathcal{H}_{q+1}^{\prime}
\end{array}
$$

commutes;

(ii) $[\beta(u), \beta(v)]^{\prime}=[u, v]$ for all $u, v \in \Pi_{q+1}$;

(iii) ${ }^{\theta}\langle u, v\rangle={ }^{\theta}\langle\gamma(u), \alpha(v)\rangle^{\prime}$ for all $u \in \mathcal{H}_{q+1}, v \in \mathcal{H}_{q}$;

$$
\begin{array}{ccc}
H_{q} & \stackrel{p_{q}}{\rightarrow} & \mathcal{H}_{q} \\
\mid \downarrow \mathcal{Z}^{a} & & \mid \downarrow \mathcal{Z}^{\alpha} \\
H_{q}^{\prime} & \stackrel{p_{q}^{\prime}}{\rightarrow} & \mathcal{H}_{q}^{\prime}
\end{array}
$$

commutes.

If (i) and (iv) hold, we say that the two $F$-forms are isomorphic.

Let $\mathscr{E}(\tilde{K})$ denote the short exact sequence of the last section: $\mathcal{H}_{q}(\tilde{K})>$ $\Pi_{q+1}(\tilde{K}) \rightarrow \mathcal{H}_{q+1}(\tilde{K})$. Similarly, let $[,]_{\tilde{K}}$ denote the Hermitian pairing and $p_{q}(\tilde{K})$ the quotient map $H_{q}(\tilde{K}) \rightarrow \mathcal{H}_{q}(\tilde{K})$. Then the work of the last section proves the following result.

THEOREM 2.1. A Z $\mathbf{Z}$-torsion-free simple $2 q-k n o t, q \geqslant 5$, with exterior $K$, gives rise to an $F$-form $\left(\mathcal{E}(\tilde{K}), H_{q}(\tilde{K}), p_{q}(\tilde{K}),[,]_{\tilde{K}},{ }^{\theta}\langle,\rangle_{\tilde{K}}\right)$.

The next two results will be established in subsequent sections.

THEOREM 2.2. For $q \geqslant 6$, two Z-torsion-free simple 2q-knots with isometric F-forms are ambient isotopic.

THeOREM 2.3. Let $\left(\mathcal{E}, H_{q}, p_{q},[],,{ }^{\theta}\langle\rangle,\right)$ be an F-form. Then for $q \geqslant 5$, there exists a Z-torsion-free simple $2 q$-knot with F-form $\left(\mathcal{E}(\tilde{K}), H_{q}(\tilde{K}), p_{q}(\tilde{K}),[,]_{\tilde{K}},{ }^{\theta}\langle,\rangle_{\tilde{K}}\right)$ isometric to the given one.

3. Algebra. An augmented $F$-form $(\mathcal{E}, \mathcal{D},[],,\langle\rangle$,$) consists of the following.$

(i) A short exact sequence of $\Gamma$-modules

$$
\mathcal{E}: \mathcal{H}_{q} \stackrel{\Omega}{\rightarrow} \Pi_{q+1} \stackrel{H}{\rightarrow} \mathcal{H}_{q+1} .
$$

(ii) Two Z-torsion-free $\Lambda$-modules of type $K, H_{q}$ and $H_{q+1}$, together with a nonsingular Hermitian pairing

$$
\langle,\rangle: H_{q+1} \times H_{q} \rightarrow \Lambda_{S} / \Lambda .
$$


(iii) A commutative diagram $\mathscr{D}$ of $\Lambda$-modules

$$
\begin{array}{rlllll} 
& 2 H_{q} & & 2 \pi_{q+1} & & 2 H_{q+1} \\
& \uparrow & & \downarrow & & \varsigma \\
2 H_{q} \rightarrow & H_{q} & \stackrel{\omega}{\rightarrow} & \pi_{q+1} & \stackrel{h_{q+1}}{\rightarrow} & H_{q+1} \\
& \downarrow p_{q} & & \downarrow^{\eta} & & \downarrow p_{q+1} \\
& \mathcal{H}_{q} & \stackrel{\Omega}{\rightarrow} & \Pi_{q+1} & \stackrel{H}{\rightarrow} & \mathcal{K}_{q+1}
\end{array}
$$

where the modules in the bottom row are regarded as $\Lambda$-modules via the ring homomorphism $\Theta: \Lambda \rightarrow \Gamma$. Each row and column is exact.

(iv) A nonsingular Hermitian pairing of $\Gamma$-modules

$$
[,]: \Pi_{q+1} \times \Pi_{q+1} \rightarrow \Gamma_{0} / \Gamma
$$

which is related to $\langle$,$\rangle by$

$$
[u, \Omega(v)]={ }^{\theta}\langle H(u), v\rangle \text { for all } u \in \Pi_{q+1}, v \in \mathcal{H}_{q},
$$

where

$$
{ }^{\theta}\langle,\rangle: \mathcal{H}_{q+1} \times \mathcal{H}_{q} \rightarrow \Gamma_{0} / \Gamma
$$

is defined by

$$
{ }^{\theta}\left\langle p_{q+1}(x), p_{q}(y)\right\rangle=\theta(\langle x, y\rangle) .
$$

Two augmented $F$-forms $A, A^{\prime}$ are isomorphic if there exist isomorphisms $a, b, c, \alpha$, $\beta, \gamma$, making the following diagram commute.

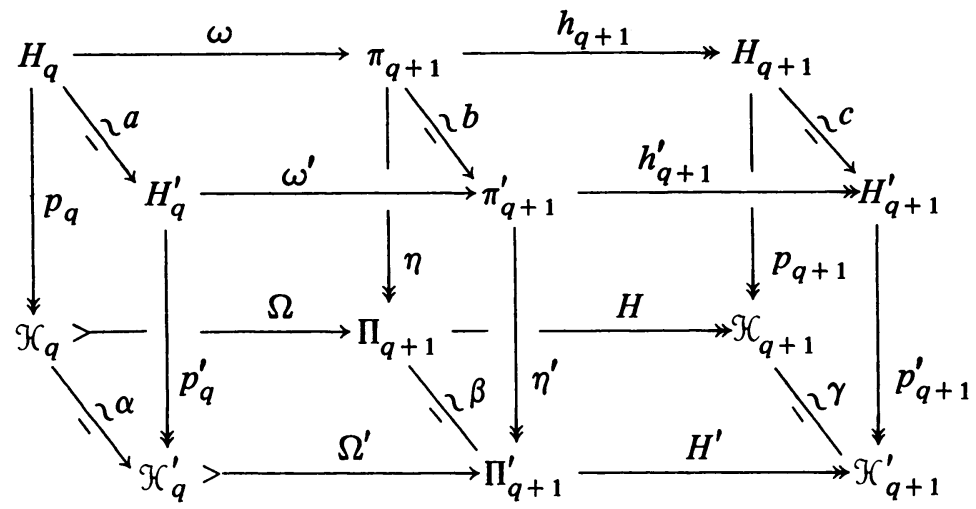

If in addition

$$
\begin{aligned}
& \langle c(x), a(y)\rangle^{\prime}=\langle x, y\rangle \text { for all } x \in H_{q+1}, y \in H_{q}, \\
& {[\beta(u), \beta(v)]^{\prime}=[u, v] \text { for all } u, v \in \Pi_{q+1},}
\end{aligned}
$$

then $A$ and $A^{\prime}$ are isometric. 
From the definitions, it is clear that each augmented $F$-form has associated with it an $F$-form, and that isometric (isomorphic) augmented $F$-forms have associated with them isometric (isomorphic) $F$-forms.

Proposition 3.1. Let $B, B^{\prime}$ be $F$-forms associated with augmented $F$-forms $A, A^{\prime}$ respectively. If $B$ is isometric (isomorphic) to $B^{\prime}$, then $A$ is isometric (isomorphic) to $A^{\prime}$.

Proposition 3.2. Let $B$ be an F-form. Then there exists an augmented $F$-form $A$ such that $B$ is associated with $A$.

The rest of this section is devoted to the proof of these two propositions.

We begin by considering an $F$-form $B=\left(\varepsilon, H_{q}, p_{q},[],,{ }^{\theta}\langle\rangle,\right)$. By [Ma, p. 90], we can regard $\mathcal{E}$ as a short exact sequence of $\Lambda$-modules, via the ring homomorphism $\Theta$ : $\Lambda \rightarrow \Gamma$. Let $H_{q+1}=\overline{\operatorname{Hom}}\left(H_{q}, \Lambda_{S} / \Lambda\right)$, and let $\langle\rangle:, H_{q+1} \times H_{q} \rightarrow \Lambda_{S} / \Lambda$ be the associated Hermitian form. By [Ke, Lemma II.12], $H_{q}$ is presented by a square nonsingular matrix, and it follows from results of Blanchfield [B, p. 351] that the pairing $\langle$,$\rangle is nonsingular. Since { }^{\theta}\langle$,$\rangle is nonsingular, there is a uniquely defined$ homomorphism $p_{q+1}: H_{q+1} \rightarrow \mathcal{H}_{q+1}$ such that

$$
{ }^{\theta}\left\langle p_{q+1}(u), p_{q}(v)\right\rangle=\theta(\langle u, v\rangle) \text { for all } u \in H_{q+1}, v \in H_{q} .
$$

LEMMA 3.1. $p_{q+1}$ is an epimorphism and $\operatorname{ker} p_{q+1}=2 H_{q+1}$.

Proof. Let $w \in \mathcal{H}_{q+1}$, and define $f \in \overline{\operatorname{Hom}}\left(H_{q}, \Gamma_{0} / \Gamma\right)$ by $f(v)={ }^{\theta}\left\langle w, p_{q}(v)\right\rangle$. The short exact sequence of $\Lambda$-modules $2 \Lambda_{S} / \Lambda \hookrightarrow \Lambda_{S} / \Lambda \rightarrow^{\theta} \Gamma_{0} / \Gamma$ induces an exact sequence

$$
\rightarrow \overline{\operatorname{Hom}}\left(H_{q}, \Lambda_{S} / \Lambda\right) \stackrel{\theta_{*}}{\rightarrow} \overline{\operatorname{Hom}}\left(H_{q}, \Gamma_{0} / \Gamma\right) \rightarrow \overline{\operatorname{Ext}}\left(H_{q}, \operatorname{ker} \theta\right) \rightarrow .
$$

By [K2, Lemma 2.3], $\overline{\operatorname{Ext}}\left(H_{q}, \operatorname{ker} \theta\right)=0$, since Z-torsion-free $\Lambda$-modules of type $K$ can be presented by square matrices. Thus there is an element $g \in \overline{\operatorname{Hom}}\left(H_{q}, \Lambda_{S} / \Lambda\right)$ such that $\theta_{*}(g)=f$. Since $\langle$,$\rangle is nonsingular, there exists u \in H_{q+1}$ such that $\langle u, v\rangle=g(v)$ for all $v \in H_{q}$, and so

$$
{ }^{\theta}\left\langle p_{q+1}(u), p_{q}(v)\right\rangle=\theta(\langle u, v\rangle)=\theta(g(v))=f(v)={ }^{\theta}\left\langle w, p_{q}(v)\right\rangle
$$

for all $v \in H_{q}$. Since $p_{q}$ is an epimorphim and $\theta\langle$,$\rangle is nonsingular, we have$ $p_{q+1}(u)=w$, and so $p_{q+1}$ is an epimorphism.

It is clear that $2 H_{q+1} \subset \operatorname{ker} p_{q+1}$, because $\Theta(2)=0$. Suppose that $p_{q+1}(u)=0$. Then for all $v \in H_{q}, \theta(\langle u, v\rangle)={ }^{\theta}\left\langle p_{q+1}(u), p_{q}(v)\right\rangle=0$, and so $\langle u, v\rangle \in \operatorname{ker} \theta=$ $2 \Lambda_{S} / \Lambda$. If $a, b \in \Lambda_{S}$ and $2 a \equiv 2 b(\bmod \Lambda)$, then $2(a-b)=c \in \Lambda$. But $a=$ $f(t) / g(t), b=h(t) / k(t)$ where $f, g, h, k \in \Lambda$ and $g(1)= \pm 1=k(1)$. So $2 \nmid g(t)$ and $2 \nmid k(t)$, which means that $2 \mid c$, and so $a-b \in \Lambda$. Therefore $a \equiv b(\bmod \Lambda)$. Thus $\langle u, v\rangle / 2$ is well defined in $\Lambda_{S} / \Lambda$ and since $\langle$,$\rangle is nonsingular, there exists$ $u^{\prime} \in H_{q+1}$ with $\left\langle u^{\prime}, v\right\rangle=\langle u, v\rangle / 2$ for all $v \in H_{q}$, and hence $u=2 u^{\prime} \in 2 H_{q+1}$.

Assume that $B$ and $B^{\prime}$ are isometric $F$-forms; then we can define an isomorphism $c: H_{q+1} \rightarrow H_{q+1}^{\prime}$ by the equation $\langle c(u), a(v)\rangle^{\prime}=\langle u, v\rangle$; this follows from the fact that $a$ is an isomorphism and $\langle\rangle,,\langle,\rangle^{\prime}$ are nonsingular. 
LEMMA 3.2. The following diagram commutes:

$$
\begin{array}{lll} 
& \stackrel{c}{\sim} \\
H_{q+1} & \stackrel{\sim}{\rightarrow} H_{q+1}^{\prime} \\
\downarrow p_{q+1} & & \downarrow p_{q+1}^{\prime} \\
& \stackrel{\gamma}{\sim} \\
\mathcal{H}_{q+1} & \stackrel{\rightarrow}{\rightarrow} \mathcal{H}_{q+1}^{\prime}
\end{array}
$$

Proof. For every $u \in H_{q+1}, v \in H_{q}$, we have

$$
\begin{aligned}
{ }^{\theta}\left\langle p_{q+1}^{\prime} c(u), p_{q}^{\prime} a(v)\right\rangle^{\prime} & =\theta\left(\langle c(u), a(v)\rangle^{\prime}\right)=\theta(\langle u, v\rangle) \\
& ={ }^{\theta}\left\langle p_{q+1}(u), p_{q}(v)\right\rangle={ }^{\theta}\left\langle\gamma p_{q+1}(u), \alpha p_{q}(u)\right\rangle^{\prime} \\
& ={ }^{\theta}\left\langle\gamma p_{q+1}(u), p_{q}^{\prime} a(v)\right\rangle^{\prime}
\end{aligned}
$$

since $\alpha p_{q}=p_{q}^{\prime} a$. As $p_{q}^{\prime} a$ is an epimorphism and ${ }^{\theta}\langle,\rangle^{\prime}$ is nonsingular, this implies that $p_{q+1}^{\prime} c=\gamma p_{q+1}$.

The short exact sequence $\mathcal{E}$ of $\Lambda$-modules and the epimorphism $p_{q+1}$ : $H_{q+1} \rightarrow \mathcal{H}_{q+1}$ yield a commutative diagram

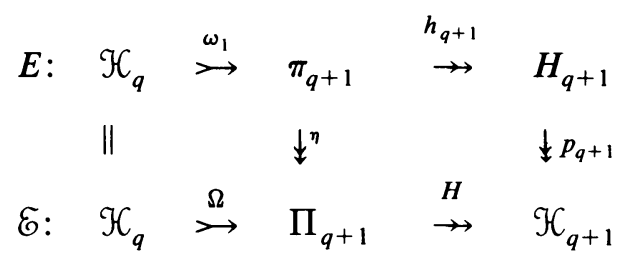

where $E$ is a short exact sequence and, in the notation of [Ma], $E=\varepsilon p_{q+1}$. An easy diagram chase shows that $\eta$ is surjective.

LEMMA 3.3. $\operatorname{ker} \eta=2 \pi_{q+1}$.

Proof. If $x \in \pi_{q+1}$, then $\eta(2 x)=2 \eta(x)=0$, since $\Pi_{q+1}$ can be regarded as a $\Gamma$-module. Thus $2 \pi_{q+1} \subset \operatorname{ker} \eta$. Conversely, suppose $x \in \operatorname{ker} \eta$. Then $p_{q+1} h_{q+1}(x)$ $=H \eta(x)=0$, and by Lemma 3.1, $h_{q+1}(x) \in 2 H_{q+1}$. Since $h_{q+1}$ is onto, there exists $y \in \pi_{q+1}$ such that $h_{q+1}(x)=2 h_{q+1}(y)$, and so $x-2 y \in \operatorname{ker} h_{q+1}=\operatorname{Im} \omega_{1}$. Thus there exists $u \in \mathscr{H}_{q}$ such that $x-2 y=\omega_{1}(u)$. Applying $\eta$, we obtain $0=$ $\eta(x)-2 \eta(y)=\Omega(u)$, and since $\Omega$ is a monomorphism, $u=0$. Therefore $x=2 y$, and so $\operatorname{ker} \eta \subset 2 \pi_{q+1}$.

We say that two short exact sequences $E$ and $E^{\prime}$ are isomorphic if there is a commutative diagram

$$
\begin{array}{llllll}
E: & A & \stackrel{\sigma}{\rightarrow} & B & \stackrel{\tau}{\rightarrow} & C \\
& \| l^{f} & & \mid l^{g} & & \mid \downarrow^{h} \\
E^{\prime}: & A^{\prime} & \stackrel{\sigma^{\prime}}{\rightarrow} & B^{\prime} & \stackrel{\tau^{\prime}}{\rightarrow} & C^{\prime}
\end{array}
$$

and write this as $E \cong E^{\prime}$. 
LEMMA 3.4. Let $E=\mathcal{E} p_{q+1}, E^{\prime}=\mathcal{E}^{\prime} p_{q+1}^{\prime}$, where $\left(\mathcal{E}, H_{q}, p_{q},[],,{ }^{\theta}\langle\rangle,\right)$ and $\left(\mathcal{E}^{\prime}, H_{q}^{\prime}, p_{q}^{\prime},[,]^{\prime},{ }^{\theta}\langle,\rangle^{\prime}\right)$ are isometric F-forms. Then $E \cong E^{\prime}$.

Proof. We have a commutative diagram:

$$
\begin{aligned}
& \text { E: } \quad \mathcal{H}_{q} \stackrel{\Omega}{\longrightarrow} \Pi_{q+1} \stackrel{H}{\rightarrow} \quad \mathcal{H}_{q+1} \\
& \left|\downarrow 2^{\alpha} \quad\right| \downarrow^{\beta} \quad \mid 2^{\gamma} \\
& \mathcal{E}^{\prime}: \quad \mathcal{H}_{q}^{\prime} \stackrel{\Omega^{\prime}}{\longrightarrow} \Pi_{q+1}^{\prime} \stackrel{H^{\prime}}{\rightarrow} \mathcal{H}_{q+1}
\end{aligned}
$$

In the notation of [Ma] this translates into $\alpha \mathcal{E}=\mathcal{E}^{\prime} \gamma$, andso $\alpha E=\alpha \delta p_{q+1}=$ $\mathcal{E}^{\prime} \gamma p_{q+1}=\mathcal{E}^{\prime} p_{q+1}^{\prime} c=E^{\prime} c$, using the fact that $\gamma p_{q+1}=p_{q+1}^{\prime} c$. Thus there is a commutative diagram

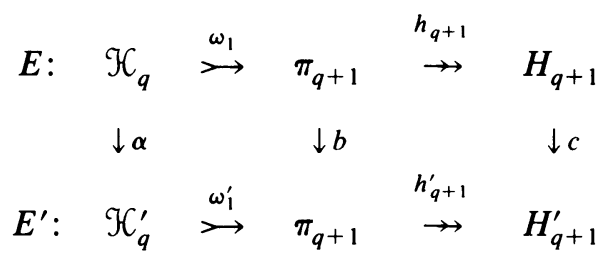

as required.

LEMMA 3.5. The isomorphism $b$ can be chosen so that the diagram

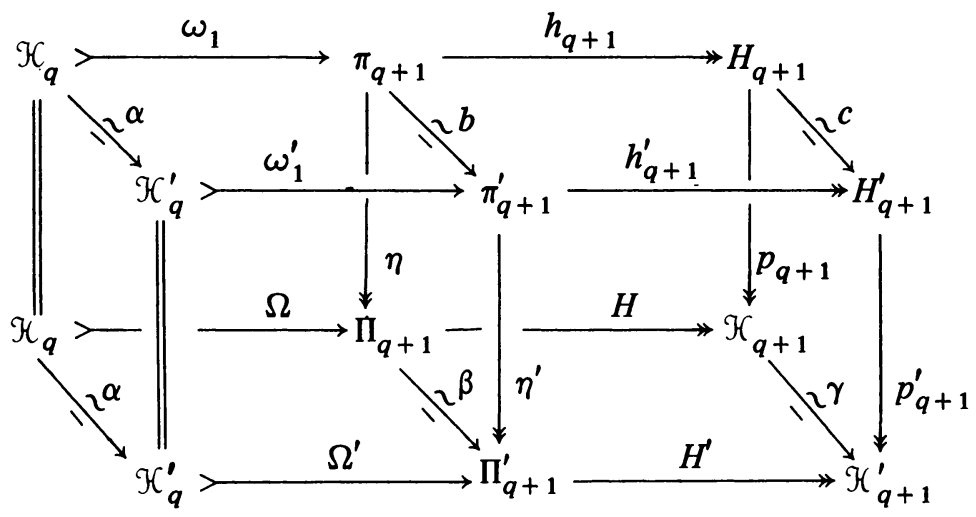

commutes.

Proof. The diagram commutes if we can choose $b$ so that $\eta^{\prime} b=\beta \eta$. First note that $H^{\prime} \eta^{\prime} b=p_{q+1}^{\prime} h_{q+1}^{\prime} b=p_{q+1}^{\prime} c h_{q+1}=\gamma p_{q+1} h_{q+1}=\gamma H \eta=H^{\prime} \beta \eta$, and so $H^{\prime}\left(\eta^{\prime} b-\beta \eta\right)=0$. Thus there is a map $\lambda: \pi_{q+1} \rightarrow \mathcal{H}_{q}^{\prime}$ such that $\Omega^{\prime} \lambda=\eta^{\prime} b-\beta \eta$. Now set $b_{1}=b-\omega^{\prime} \lambda$; then $\eta^{\prime} b_{1}-\beta \eta=\eta^{\prime} b-\eta^{\prime} \omega_{1}^{\prime} \lambda-\beta \eta=\eta^{\prime} b-\Omega^{\prime} \lambda-\beta \eta=0$. It remains to check that when $b_{1}$ is substituted for $b$, the other parts of the diagram above still commute: the five-lemma will then imply that $b_{1}$ is an isomorphism. 
First note that

$$
h_{q+1}^{\prime} b_{1}=h_{q+1}^{\prime} b-h_{q+1}^{\prime} \omega_{1}^{\prime} \lambda=h_{q+1}^{\prime} b=c h_{q+1} .
$$

In particular, $b_{1}\left(\operatorname{ker} h_{q+1}\right) \subset \operatorname{ker} h_{q+1}^{\prime}$, and so $\operatorname{Im}\left(b_{1} \omega_{1}\right) \subset \operatorname{Im}\left(\omega_{1}^{\prime} \alpha\right)=\operatorname{Im} \omega_{1}^{\prime}$. Since $\eta^{\prime} \omega_{1}^{\prime}=\Omega^{\prime}, \eta^{\prime}$ restricted to $\operatorname{Im} \omega_{1}^{\prime}$ is a monomorphism. But $b_{1} \omega_{1}-\omega_{1}^{\prime} \alpha=b_{1} \omega_{1}-b \omega_{1}$, so $\operatorname{Im}\left(b_{1} \omega_{1}-b \omega_{1}\right) \subset \operatorname{Im} \omega_{1}^{\prime}$. Moreover, $\eta^{\prime} b \omega_{1}=\eta^{\prime} \omega_{1}^{\prime} \alpha=\Omega^{\prime} \alpha=\beta \Omega=\beta \eta \omega_{1}=$ $\eta^{\prime} b_{1} \omega_{1}$, so $\eta^{\prime}\left(b_{1} \omega_{1}-b \omega_{1}\right)=0$. Since $\eta^{\prime} \mid \operatorname{Im} \omega_{1}^{\prime}$ is a monomorphism, we have $b_{1} \omega_{1}=$ $b \omega_{1}=\omega_{1}^{\prime} \alpha$.

Define $\omega: H_{q} \rightarrow \pi_{q+1}$ by $\omega=\omega_{1} p_{q}$; since $\omega_{1}$ is a monomorphism, $\operatorname{ker} \omega=\operatorname{ker} p_{q}$ $=2 H_{q}$. Starting from an $F$-form, we have now constructed a commutative diagram $\mathscr{D}$ as in (3.1), in which the rows and columns are exact.

The $\Lambda$-module $H_{q}$ has a presentation by a square matrix $M$ say [Ke, Lemma II.12], and so by $[\mathrm{B}, \S 4], H_{q+1}=\overline{\operatorname{Hom}}\left(H_{q}, \Lambda_{S} / \Lambda\right)$ has a presentation by $M^{*}$, and so $H_{q+1}$ is a Z-torsion-free $\Lambda$-module of type $K$. We have therefore proved Proposition 3.2 and Proposition 3.1.

Finally we define a pairing

$$
\{,\}: \pi_{q+1} \times \pi_{q+1} \rightarrow \Gamma_{0} / \Gamma
$$

by

$$
\{x, y\}=[\eta(x), \eta(y)] .
$$

LEMMA 3.6. The isomorphism $b$ is an isometry of $\{$,$\} .$

Proof. $\{b(x), b(y)\}^{\prime}=\left[\eta^{\prime} b(x), \eta^{\prime} b(y)\right]^{\prime}=[\beta \eta(x), \beta \eta(y)]^{\prime}=[\eta(x), \eta(y)]=$ $\{x, y\}$.

LEMMA 3.7. For all $x \in \pi_{q+1}, v \in H_{q}$, we have

$$
\{x, \omega(v)\}=\theta\left(\left\langle h_{q+1}(u), v\right\rangle\right) .
$$

Proof. $\{x, \omega(v)\}=[\eta(x), \eta \omega(v)]=\left[\eta(x), \eta \omega_{1} p_{q}(v)\right]=\left[\eta(x), \Omega p_{q}(v)\right]=$ ${ }^{\theta}\left\langle H \eta(x), p_{q}(v)\right\rangle={ }^{\theta}\left\langle p_{q+1} h_{q+1}(x), p_{q}(v)\right\rangle=\theta\left(\left\langle h_{q+1}(x), v\right\rangle\right)$.

4. Matrix presentations. We begin this section by considering a sequence of $\Lambda$-modules

$$
\Lambda^{n} \stackrel{d_{2}}{\rightarrow} \Lambda^{m+n} \stackrel{d_{1}}{\rightarrow} \Lambda^{m} \rightarrow H_{q}
$$

which is exact at $\Lambda^{m}$, with $d_{1} d_{2}=0$, and the short exact sequence

$$
\Lambda^{n} \stackrel{d_{2}}{\rightarrow} \operatorname{ker} d_{1} \rightarrow H_{q+1} \text {. }
$$

We assume that $H_{q}$ and $H_{q+1}$ are $\Lambda$-torsion-modules, and shall make further assumptions later in the section.

LEMMA 4.1. $\operatorname{ker} d_{1}$ is projective.

Proof. The sequence

$$
\operatorname{ker} d_{1}>\Lambda^{m+n} \stackrel{d_{1}}{\rightarrow} \Lambda^{m} \rightarrow H_{q}
$$


is exact. Now $\Lambda=\mathbf{Z}[t]_{S}$ where $S=\left\{t^{i}: 0 \leqslant i<\infty\right\}$, and so by [N, $\$ 9.2$, Theorem 8] we have gl.dim $\Lambda \leqslant \operatorname{gl} \cdot \operatorname{dim} \mathbf{Z}[t]=2$. Therefore $\operatorname{ker} d_{1}$ is projective.

LEMMA 4.2. Every finitely-generated projective $\Lambda$-module is stably-free.

Proof. By [Ba, p. 636], $K_{0}(\Lambda)=K_{0}\left(\mathbf{Z}\left[t, t^{-1}\right]\right) \cong K_{0}(\mathbf{Z})$, and by [M, p. 7], $K_{0}(\mathbf{Z}) \cong$ $\mathbf{Z}$, since $\mathbf{Z}$ is a principal ideal domain. Thus every finitely-generated projective $\Lambda$-module is stably-free.

COROllary 4.3. After stabilising, we may assume that $\operatorname{ker} d_{1}$ is free in the sequences (4.1) and (4.2).

Proof. By adding $\Lambda^{r} \stackrel{1}{\rightarrow} \Lambda^{r}$ to $\Lambda^{n} \stackrel{d_{2}}{\longrightarrow} \Lambda^{m+n}$ for sufficiently large $r$ we obtain a sequence

$$
\Lambda^{n^{\prime}} \stackrel{d_{2}^{\prime}}{\longrightarrow} \Lambda^{m+n^{\prime}} \stackrel{d_{1}^{\prime}}{\rightarrow} \Lambda^{m} \rightarrow H_{q}
$$

where $n^{\prime}=n+r, d_{2}^{\prime}=d_{2} \oplus 1, \operatorname{ker} d_{1}^{\prime}=\operatorname{ker} d_{1} \oplus \Lambda^{r} \cong \Lambda^{m+r}$. This sequence is exact except at $\Lambda^{m+n^{\prime}}$, and (4.2) is replaced by

$$
\Lambda^{n^{\prime}} \stackrel{d_{2}^{\prime}}{\longrightarrow} \operatorname{ker} d_{1}^{\prime} \rightarrow H_{q+1}
$$

From now on we assume that $H_{q}$ and $H_{q+1}$ are $\mathbf{Z}$-torsion-free $\Lambda$-modules of type $K$.

LEMMA 4.4. After stabilising, we may assume that $\mathrm{ker} d_{1}$ is a free direct summand of $\Lambda^{m+n}$.

Proof. By the previous result, we may take ker $d_{1}$ to be free. By [Ke, Lemma II.12], there is a short exact sequence

$$
\Lambda^{r} \longrightarrow \Lambda^{r} \rightarrow H_{q}
$$

and so the homological dimension of $H_{q}$ is at most 1. Alternatively, see [L, Proposition 3.5]. But we have an exact sequence

$$
\Lambda^{m+n} / \operatorname{ker} d_{1} \longrightarrow \Lambda^{m} \rightarrow H_{q},
$$

and so $\Lambda^{m+n} / \operatorname{ker} d_{1}$ is projective. By Lemma 4.2 , it is therefore stably-free. So there is an exact sequence

$$
\operatorname{ker} d_{1} \longrightarrow \Lambda^{m+n} \stackrel{\tau}{\rightarrow} \Lambda^{m+n} / \operatorname{ker} d_{1}
$$

with the first two modules free and the third stably-free.

Adding $\Lambda^{k} \stackrel{1}{\rightarrow} \Lambda^{k}$ to $\Lambda^{m+n} \stackrel{d_{1}}{\rightarrow} \Lambda^{m}$ keeps the original sequence (4.1) exact at $\Lambda^{m}$, and for sufficiently large $k$ makes $\Lambda^{m+n} / \operatorname{ker} d_{1}$ free. The sequence (4.2) is not affected, and (4.3) becomes

$$
\operatorname{ker} d_{1}>\Lambda^{m+n} \oplus \stackrel{\tau \oplus \mathrm{I}}{\rightarrow}\left(\Lambda^{m+n} / \operatorname{ker} d_{1}\right) \oplus \Lambda^{k}
$$


which is a short exact sequence of free $\Lambda$-modules, and hence is split. This proves the result.

CoROLlaRy 4.5. Writing $\Lambda^{m+n}=\Lambda^{m} \oplus \Lambda^{n}$, we can arrange that the sequence (4.1) decomposes into the short exact sequences

$$
\Lambda^{n} \stackrel{d_{1}}{\rightarrow} \Lambda^{n} \rightarrow H_{q+1}, \quad \Lambda^{m} \stackrel{d_{2}}{\longrightarrow} \Lambda^{m} \rightarrow H_{q}
$$

Let $A$ be an $n \times n$ matrix over $\Lambda$. We shall be concerned with the following operations on $A$.

(i) Multiply a row (or column) of $A$ by $\pm t^{i}$.

(ii) Permute the rows (or columns) of $A$.

(iii) Add one row (or column) to another row (or column).

(iv) Replace $A$ by the $(n+1) \times(n+1)$ matrix $\left(\begin{array}{ll}A & 0 \\ 0 & 1\end{array}\right)$.

(v) The inverse of (iv).

An $n \times n$ matrix $N$ over $\Lambda$ is called elementary if either (a) all the diagonal entries are 1 , except for one which is $\pm t^{i}$, and all off-diagonal entries are 0 ; or (b) all the diagonal entries are 1 and there is just one nonzero off-diagonal entry, which is 1 . Note that an elementary matrix $N$ is invertible over $\Lambda$, for $\operatorname{det} N= \pm t^{i}$ which is a unit in $\Lambda$.

LEMMA 4.6. Let $N$ be an invertible matrix over $\Lambda$. Then $\left(\begin{array}{ll}N & 0 \\ 0 & I\end{array}\right)$ can be written as a product of elementary matrices, for some identity matrix $I$.

Proof. $\Lambda$ is the group ring of the infinite cyclic group $(t:)$, of which the Whitehead group $\mathrm{Wh}((t:))$ is trivial. The result follows at once.

Elementary matrices are of interest here because they correspond to the operations (i) and (iii) listed above. For example, if $N$ is an elementary matrix of type (b), then $A \mapsto A N$ corresponds to a column operation of type (iii). The matrix operation (ii) is redundant, being a consequence of operations (i) and (iii), but it is convenient in practice.

Two $\Lambda$-matrices $A$ and $B$ are equivalent $(A \sim B)$ if one can pass from $A$ to $B$ by a finite sequence of operations (i)-(v). Clearly $\sim$ is an equivalence relation.

The matrix $A$ presents the $\Lambda$-module $M$ if there is an exact sequence

$$
\Lambda^{m} \stackrel{\alpha}{\rightarrow} \Lambda^{n} \stackrel{\phi}{\rightarrow} M
$$

where the map $\alpha$ is represented by $A$ with respect to some bases $x_{1}, \ldots, x_{n}$ of $\Lambda^{n}$ and $z_{1}, \ldots, z_{m}$ of $\Lambda^{m}$; that is,

$$
\alpha\left(z_{i}\right)=\sum_{j=1}^{n} A_{i j} x_{j}, \quad 1 \leqslant i \leqslant m .
$$

Lemma 4.7. Let the matrix $A$ present the $\Lambda$-module $M$, and the matrix $B$ present the $\Lambda$-module $N$. Assume that both matrices are square, with nonzero determinants. Then $M \cong N \Leftrightarrow A \sim B$. 
Proof. The operations (i)-(iii) on $A$ correspond to changes of basis in the free modules of (4.4), whilst (iv) corresponds to replacing (4.4) by

$$
\Lambda^{m} \oplus \stackrel{\alpha \oplus 1}{\rightarrow} \Lambda^{m} \oplus \Lambda \stackrel{(\phi, 0)}{\rightarrow} M .
$$

Thus $A \sim B \Rightarrow M \cong N$.

Conversely, suppose that $f: M \rightarrow N$ is an isomorphism. We have short exact sequences

$$
F \stackrel{\alpha}{\longrightarrow} G \stackrel{\phi}{\rightarrow} M, \quad H \stackrel{\beta}{\longrightarrow} L \stackrel{\psi}{\rightarrow} N
$$

with $F \cong G \cong \Lambda^{m}, H \cong L \cong \Lambda^{n}$. The map $\alpha$ is a monomorphism because det $A \neq 0$, and similarly for $\beta$. Let $x_{1}, \ldots, x_{m}$ be the basis of $G$ and $x_{m+1}, \ldots, x_{m+n}$ the basis of $L$. By moves of type (iv), these exact sequences may be replaced by

$$
\begin{array}{lllll}
F \oplus L & \stackrel{\alpha \oplus 1}{\rightarrow} & G \oplus L & \stackrel{(\phi, 0)}{\rightarrow} & M, \\
& & & & \\
G \oplus H & \stackrel{1 \oplus \beta}{\rightarrow} & G \oplus L & \stackrel{(0, \psi)}{\rightarrow} & N
\end{array}
$$

where $G \oplus L$ has basis $x_{1}, \ldots, x_{m+n}$. To save notation, we write these as

$$
\begin{array}{lllll}
F & \stackrel{\alpha}{\longrightarrow} & G & \stackrel{\phi}{\rightarrow} & M, \\
H & \stackrel{\beta}{\longrightarrow} & L & \stackrel{\psi}{\rightarrow} & N
\end{array}
$$

where $F \cong G \cong H \cong L \cong \Lambda^{m+m} ; x_{1}, \ldots, x_{m+n}$ is the basis of $G ; y_{1}, \ldots, y_{m+n}$ is the basis of $L$, and

$$
\begin{array}{rlrl}
\phi x_{i} & =0, & & m<i \leqslant m+n, \\
\psi y_{i} & =0, & i \leqslant i \leqslant m .
\end{array}
$$

As $\psi$ is surjective, and $\psi y_{i}=0$, there exist $k_{m+1}, \ldots, k_{m+n} \in \Lambda$ such that

$$
f \phi x_{1}=\psi y_{1}+k_{m+1} \psi y_{m+1}+\cdots+k_{m+n} \psi y_{m+n} .
$$

Replacing $y_{1}$ by $y_{1}+k_{m+1} y_{m+1}+\cdots+k_{m+n} y_{m+n}$ in the basis of $L$, we may arrange that $f \phi x_{1}=\psi y_{1}$. Continuing in this way, we change basis in $L$ so that

$$
f \phi x_{i}=\psi y_{i}, \quad 1 \leqslant i \leqslant m .
$$

Similarly, by changing basis in $G$ we may arrange that

$$
f \phi x_{i}=\psi y_{i}, \quad m<i \leqslant m+n,
$$

and hence $f \phi x_{i}=\psi y_{i}, 1 \leqslant i \leqslant m+n$.

Note that this is achieved by means of column operations of type (iii) on $A$ and $B$.

Let $g: G \rightarrow L$ be the $\Lambda$-module isomorphism defined by $g x_{i}=y_{i}$ for $1 \leqslant i \leqslant m+n$. Then $f \phi=\psi g$, and in particular $g$ restricts to an isomorphism from $\operatorname{ker} \phi=\operatorname{Im} \alpha$ onto $\operatorname{ker} \psi=\operatorname{Im} \beta$. Thus $g$ determines a unique isomorphism $h$ such that the following diagram commutes. 


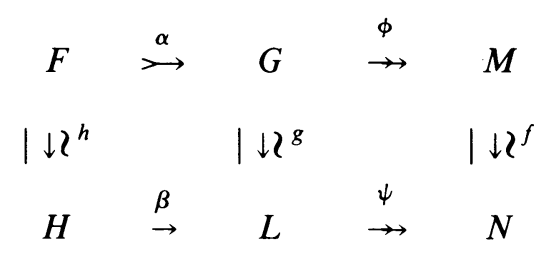

Changing basis in $H$ so that (basis of $H$ ) $=h$ (basis of $F$ ) gives an invertible matrix $E$ such that $A=E B$. By Lemma 4.6 we can, after operations of type (iv) if necessary, assume that $E$ is a product of elementary matrices, and so $A$ and $B$ are connected by a sequence of operations of types (i), (iii), (iv) and (v). Thus $A \sim B$.

5. Isotopy. In this section, $Q$ is a compact manifold of dimension $2 q+1$, with $\partial Q \cong M \times S^{1}$. Let $r$ be an integer satisfying $3 q \leqslant 3 r \leqslant 4 q-3$.

Let $f: B^{r} \times B^{2 q-r} \rightarrow Q$ be an embedding which takes $\partial B^{r} \times B^{2 q-r}$ into $M=M$ $\times 0 \subset \partial Q$, for some fixed point $0 \in S^{1}$, and the rest of $B^{r} \times B^{2 q-r}$ into int $Q$. Such an embedding is called permissible. An ambient isotopy of $f$ is permissible if it is an isotopy through permissible embeddings. Let $M \times B^{1} \subset \partial Q$; then a permissible embedding $f$ extends to an embedding $f^{\prime}: B^{r} \times B^{2 q-r} \times B^{1} \rightarrow Q$ such that $\operatorname{Im} f^{\prime}$ is a regular neighbourhood of $\operatorname{Im} f$ rel $f\left(B^{r} \times \partial B^{2 q-r}\right)$ meeting $\partial Q$ regularly, with $f^{\prime} \mid \partial B^{r} \times B^{2 q-r} \times B^{1}: \quad \partial B^{r} \times B^{2 q-r} \times B^{1} \rightarrow M \times B^{1}$ being $\left(f \mid \partial B^{r} \times B^{2 q-r}\right) \times$ identity. A permissible ambient isotopy of $f$ extends to a permissible ambient isotopy of $f^{\prime}$; that is, an isotopy of $f^{\prime}$ through embeddings of the same form as $f^{\prime}$.

Let $f^{\prime \prime}=f^{\prime} \mid B^{r} \times 0 \times\{0,1\}$; for convenience we write this as $f^{\prime \prime}: B^{r} \times \partial I \rightarrow Q$. Let $g, g^{\prime}, g^{\prime \prime}$ be similarly defined, with $g=f$ on the subset

$$
\left(B^{r} \times 0\right) \cup\left(\partial B^{r} \times B^{2 q-r}\right) .
$$

Then $g^{\prime}=f^{\prime}$ on the subset $\left(B^{r} \times 0 \times 0\right) \cup\left(\partial B^{r} \times B^{2 q-r} \times B^{1}\right)$, and we can take $\operatorname{Im} g^{\prime}=\operatorname{Im} f^{\prime}$ by the uniqueness of regular neighbourhoods. A permissible ambient isotopy $F^{\prime \prime}$ of $f^{\prime \prime}$ to $g^{\prime \prime}$ is one which

(i) restricts to a permissible ambient isotopy of $f^{\prime \prime} \mid B^{r} \times 0$ to $g^{\prime \prime} \mid B^{r} \times 0$;

(ii) for every $t \in I$, has $p F_{t}^{\prime \prime}(x, 1)=F_{t}^{\prime \prime}(x, 0)$ where $p: M \times B^{1} \rightarrow M \times 0$ is projection along $B^{1}$;

(iii) $F^{\prime \prime}\left(B^{r} \times 1 \times I\right)$ is contained in a regular neighbourhood of $F^{\prime \prime}\left(B^{r} \times 0 \times I\right)$ which meets $Q \times \partial I$ in $\operatorname{Im} f^{\prime} \cup \operatorname{Im} g^{\prime}$, and meets $\partial(Q \times I)$ regularly.

In practice we shall take the regular neighbourhood in (iii) to be the trace of an isotopy of $\operatorname{Im} f^{\prime}$ to $\operatorname{Im} g^{\prime}$; this is possible by the uniqueness of regular neighbourhoods up to ambient isotopy.

A permissible homotopy $F^{\prime \prime}: B^{r} \times \partial I \times I \rightarrow Q \times I$ is one in which the conditions (i), (ii) and (iii) hold; so that $F^{\prime \prime}$ is an isotopy on $B^{r} \times 0$ and on $\partial B^{r} \times \partial I$.

Let $\pi^{s}$ denote the stable $s$-stem of the sphere, so that $\pi^{s} \cong \pi_{n+s}\left(S^{n}\right)$ for large $n$.

Lemma 5.1. The permissible homotopy $F^{\prime \prime}$ of $f^{\prime \prime}$ to $g^{\prime \prime}$ is homotopic (rel d) to a permissible isotopy of $f^{\prime \prime}$ to $g^{\prime \prime}$ if and only if an obstruction $d\left(f^{\prime \prime}, g^{\prime \prime} ; F^{\prime \prime}\right) \in \pi^{2(r-q)}$ vanishes. 
Proof. Let $N$ be the regular neighbourhood of $F^{\prime \prime}\left(B^{r} \times 0 \times I\right)$ in $Q \times I$ referred to in (iii). Then $N$ is a $(2 q+2)$-ball since $F^{\prime \prime} \mid B^{r} \times 0 \times I$ is an embedding, and $F^{\prime \prime} \mid B^{r} \times 1 \times I: B^{r} \times 1 \times I \rightarrow N$ is an embedding on the boundary by (ii). This is homotopic (rel $\partial$ ) to a proper embedding, and so we have a proper map $G$ : $B^{r} \times \partial I \times I \rightarrow N$ which is an embedding on each component. The results of [K1, §2] yield an obstruction in $\pi^{2(r-q)}$ to homotoping $G$ (rel $\partial$ ) to an embedding, and this obstruction we denote by $d\left(f^{\prime \prime}, g^{\prime \prime} ; F^{\prime \prime}\right)$. (Strictly speaking, the obstruction is a matrix

$$
\left(\begin{array}{cc}
0 & x \\
(-1)^{r+1} & 0
\end{array}\right),
$$

and we are taking $d\left(f^{\prime \prime}, g^{\prime \prime} ; F^{\prime \prime}\right)= \pm x$. The sign will not be important here, and we shall not trouble to keep track of it.)

If $d\left(f^{\prime \prime}, g^{\prime \prime}, F^{\prime \prime}\right)=0$, then as in $[\mathbf{K 1}, \S 2]$ we may homotop $G$ (rel $\partial$ ) to an embedding. Since the codimension is $2 q+2-(r+1)=2 q-r+1 \geqslant 2(r-q)+$ $6>3$, Hudson's theorems on "concordance implies isotopy" enable us to homotop $G$ (rel $\partial$ ) to a permissible isotopy. The converse is implied by [K1, §2].

LEMMA 5.2. If $d\left(f^{\prime \prime}, g^{\prime \prime} ; F^{\prime \prime}\right)=0$, then $f$ is permissibly ambient isotopic to $g$.

Proof. By the previous lemma, we may take $F^{\prime \prime}$ to be a permissible ambient isotopy. Since

$$
X=\operatorname{cl}(\partial N \cap(\operatorname{Int}(Q \times I)))
$$

is a deformation retract of $N-F^{\prime \prime}\left(B^{r} \times 0 \times I\right)$, we can homotop $F^{\prime \prime} \mid B^{r} \times 1 \times I$ (rel $\partial$ ) to a map into $X$. Now $X \cong S^{2 q-r} \times B^{r+1}$, and so by Irwin's Theorem this map is homotopic (rel $\partial$ ) to an embedding of $B^{r} \times 1 \times I$ in $X$; the inequalities to check are

$$
2(r+1)-(2 q+1)+1 \leqslant 2 q-r-1,
$$

that is $3 r \leqslant 4 q-3$, and $2 q+1-(r+1) \geqslant 3$, that is $2 q-r \geqslant 3$, both of which are satisfied. Using Hudson's results on "concordance implies isotopy" again, this embedding can be moved to a permissible isotopy, so we can assume that $F^{\prime \prime}$ keeps $B^{r} \times 1$ in the boundary of a regular neighbourhood of $B^{r} \times 0$. By the Alexander trick, $F^{\prime \prime}$ extends to an isotopy $F^{\prime}$ taking $f^{\prime} \mid B^{r} \times 0 \times[0,1]$ to $g^{\prime} \mid B^{r} \times 0 \times[0,1]$. Since $f^{\prime}\left(B^{r} \times B^{2 q-r} \times[0,1]\right)$ is a regular neighbourhood of $f^{\prime}\left(B^{r} \times 0 \times[0,1]\right)$ rel $\partial f^{\prime}\left(B^{r} \times 0 \times[0,1]\right)$, and so is $g^{\prime}\left(B^{r} \times B^{2 q-r} \times[0,1]\right)$, there is an ambient isotopy $F$ extending $F^{\prime}$ and taking the one set onto the other. The Alexander trick then shows that $f^{\prime} \mid B^{r} \times B^{2 q-r} \times[0,1]$ is ambient isotopic to $g^{\prime} \mid B^{r} \times B^{2 q-r} \times[0,1]$, and hence that $f$ is permissibly ambient isotopic to $g$.

LEMMA 5.3. Let $f, g: B^{r} \times B^{2 q-r} \rightarrow Q$ be permissible embeddings which agree on $\left(B^{r} \times 0\right) \cup\left(\partial B^{r} \times B^{2 q-r}\right)$. Assume that there is a ball $B^{2 q-r+1}$ properly embedded in $Q$, with $\partial B^{2 q-r+1} \subset M \times 0 \subset \partial Q$, such that $\partial B^{2 q-r+1}$ meets $f\left(\partial B^{r} \times 0\right)$ transversely in $M$ in a single point, and that this is the only point of intersection of $B^{2 q-r+1}$ and $f\left(B^{r}\right)$. Then there is a permissible ambient isotopy taking $f$ to $g$. 
Proof. With the notation above, let $F^{\prime \prime}: B^{r} \times \partial I \times I \rightarrow Q \times I$ be a permissible homotopy of $f^{\prime \prime}$ to $g^{\prime \prime}$, and let $v=d\left(f^{\prime \prime}, g^{\prime \prime} ; F^{\prime \prime}\right)$. We shall alter $F^{\prime \prime}$ to a permissible homotopy $G^{\prime \prime}$ such that $d\left(f^{\prime \prime}, g^{\prime \prime} ; G^{\prime \prime}\right)=0$.

Let $F=F^{\prime \prime} \mid B^{r} \times 0 \times I$. Let $k: B^{2 q-r+1} \rightarrow Q \times \frac{1}{2}$ be the inclusion map, and let $x \in k\left(\partial B^{2 q-r+1}\right) \cap F\left(\partial B^{r} \times 0 \times \frac{1}{2}\right)$. We can assume that $F^{\prime \prime}$ is the constant homotopy on $\left[0, \frac{3}{4}\right] \times I$, and hence that $x=\operatorname{Im} k \cap \operatorname{Im} F$. Let $T \cong B^{2 q+2}$ be a regular neighbourhoood of $x$ in $Q \times I$, meeting $\partial Q \times I, \operatorname{Im} k$ and $\operatorname{Im} F$ regularly, and missing $Q \times \partial I$. Let $P=T \cap(M \times 0 \times I) \subset T \cap(\partial Q \times I)$, which we may assume to be a $2 q$-ball. Then $F \mid F^{-1}(\partial P)$ is an embedding of $S^{r-1}$ in $\partial P \cong S^{2 q-1}$ and $k \mid k^{-1}(\partial P)$ is an embedding of $S^{2 q-r-1}$ in $\partial P$. The linking number of these two embeddings is \pm 1 , because of the single transverse intersection point $x$ in $P$.

Let $u \in \pi_{r-1}\left(S^{2 q-r-1}\right)$ correspond to $v \in \pi^{2(r-q)}$, and let

$$
B_{1}^{2 q-r+1}=\operatorname{cl}\left[B^{2 q-r-1}-k^{-1}(T)\right] .
$$

Note that $k^{-1}(\partial P)$ is a $(2 q-r-1)$-sphere in $\partial B_{1}^{2 q-r+1}$. Let $h: S^{r-1} \rightarrow \partial P \cap \operatorname{Im} k$ $\cong S^{2 q-r-1}$ represent $u$. By suspending, extend $h$ to $h: S^{r} \rightarrow k\left(\partial B_{1}^{2 q-r+1}\right)$, and then extend to $h: B^{r+1} \rightarrow k\left(B_{1}^{2 q-r+1}\right)$ by coning. Note that $h^{-1}(\partial T)$ is an $r$-ball in $\partial B^{r+1}$.

Consider $h \mid S^{r-1}$ as a map into a regular neighbourhood of $\partial P \cap \operatorname{Im} k$ in $\partial P$; this is homeomorphic to $S^{2 q-r-1} \times B^{r}$. By Irwin's Theorem, this map is homotopic to an embedding; the inequalities to check are $2(r-1)-(2 q-1)+1 \leqslant 2 q-r-2$, that is $3 r \leqslant 4 q-2$, and $2 q-1-(r-1) \geqslant 3$, that is $2 q-r \geqslant 3$. By suspension and coning we can extend this to a homotopy which takes $h: B^{r+1} \rightarrow Q \times I-$ int $T$ to an embedding. We now have disjoint embeddings $F \mid F^{-1}(\partial P): S^{r-1} \rightarrow \partial P \cong$ $S^{2 q-1}$, and $h \mid S^{r-1}: S^{r-1} \rightarrow \partial P$, and the linking element of these is $\pm u \in$ $\pi_{r-1}\left(S^{2 q-r-1}\right)$. This embedding of $S^{r-1} \cup S^{r-1}$ in $\partial P$ extends to an embedding $\alpha$ of $S^{r-1} \times I$ in $\partial P$, for we can certainly find an embedding $\alpha$ of $S^{r-1} \times I$ in $\partial P$ such that $\alpha\left(S^{r-1} \times O\right)$ and $\alpha\left(S^{r-1} \times 1\right)$ have linking element $\pm u$, and in these dimensions homotopy implies isotopy (the inequalities needed are $(2 q-r-1)-(r-1)$ $\geqslant 3$, that is $2(q-r) \geqslant 3,2(r-1)-(2 q-r-1)+1 \leqslant r-2$, that is $2 q-r \geqslant 2$, and $2(r-1)-(2 q-r-1)+2 \leqslant 2 q-r-2$, that is $3 r \leqslant 4 q-3)$. Thus we have an embedding of

$$
S^{r}=B^{r} \cup\left(S^{r-1} \times I\right) \cup B^{r}
$$

into $\partial T$ given by $\alpha$, the restriction of $h$ to $h^{-1}(\partial T) \cong B^{r}$ and the restriction of $F$ to $F^{-1}(\partial T) \cong B^{r}$. By coning, this extends to an embedding of $B^{r+1}$ into $T$, and hence we have an embedding $G$ of $\left(B^{r} \times I\right) \#_{\partial} B^{r+1}$ into $Q \times I$, where ${ }_{\partial}$ denotes the boundary connected sum.

Now $\left(B^{r} \times I\right) \#_{\partial} B^{r+1} \cong B^{r} \times I$, and $G \mid \partial B^{r} \times I: \partial B^{r} \times I \rightarrow M \times I$ is a concordance of $f \mid \partial B^{r} \times 0$ and $g \mid \partial B^{r} \times 0$. Keeping the ends fixed, we can by Hudson's results straighten this up to be an isotopy. By a collaring argument, we obtain a concordance $G: B^{r} \times I \rightarrow Q \times I$ which restricts to an isotopy of $\partial B^{r} \times I \rightarrow M \times I$. Again using Hudson's results, we can straighten $G$ up so that it is an isotopy of $f \mid B^{r} \times 0$ to $g \mid B^{r} \times 0$ without disturbing the boundary.

The map $k: B^{2 q-r+1} \rightarrow Q \times \frac{1}{2}$ extends to an embedding $k: B^{2 q-r+1} \times B^{1} \rightarrow Q$ $\times \frac{1}{2}$ such that $k \mid \partial B^{2 q-r+1} \times B^{1}: \partial B^{2 q-r+1} \times B^{1} \rightarrow M \times B^{1} \times \frac{1}{2}$ is of the form 
$\left(k \mid \partial B^{2 q-r+1}\right) \times$ identity. We can arrange that $k\left(\partial\left(B^{2 q-r+1} \times B^{1}\right)\right)$ meets $\operatorname{Im} F$ in just two points, one of these being $x$.

The boundary connected sum operation can be repeated on $F^{\prime \prime} \mid B^{r} \times 1 \times I$ and $k \mid B^{2 q-r+1} \times 1$ to yield a permissible homotopy $G^{\prime \prime}$ from $f^{\prime \prime}$ to $g^{\prime \prime}$. From the remarks above, the set $F^{\prime \prime}\left(B^{r} \times 1 \times I\right) \cup k\left(B^{2 q-r+1} \times 1\right)$ meets $F^{\prime \prime}\left(B^{r} \times 0 \times I\right)$ $\cup k\left(B^{2 q-r+1} \times 0\right)$ transversely in a single point. As in the proof of [K1, Proposition $3.2]$, it follows that $d\left(f^{\prime \prime}, g^{\prime \prime} ; G^{\prime \prime}\right)=d\left(f^{\prime \prime}, g^{\prime \prime} ; F^{\prime \prime}\right) \pm v$, and so by choosing the orientation of $k$ correctly we can ensure that $d\left(f^{\prime \prime}, g^{\prime \prime} ; G^{\prime \prime}\right)=0$. Lemma 5.2 completes the proof.

6. Geometry. Suppose that $1<r<n$, and consider a handle decomposition of $S^{n}$ with one 0 -handle $B^{n}, k(r-1)$-handles $h_{i}^{r-1}(1 \leqslant i \leqslant k), k r$-handles $h_{i}^{r}(1 \leqslant i \leqslant k)$, and one $n$-handle. Assume that $h_{i}^{r}$ cancels $h_{i}^{r-1}$ for each $i$, and that the $n$-balls $h_{i}^{r-1} \cup h_{i}^{r}$ are pairwise disjoint. Let $X=B^{n} \cup \cup_{i=1}^{n} h_{i}^{r-1}$. Then $\partial X$ has a regular neighbourhood in $S^{n}$ of the form $\partial X \times B^{1}$, and from the handle decomposition above we obtain a handle decomposition of $S^{n}$ on $\partial X \times B^{1}$ with $k r$-handles, $k$ $(n-r+1)$-handles, and two $n$-handles.

Embed $S^{n}$ in $S^{n+1}$ as the equatorial $n$-sphere. Then $S^{n}$ has a regular neighbourhood of the form $S^{n} \times B^{1}$, and so $\partial X$ has a regular neighbourhood in $S^{n+1}$ of the form $\partial X \times B^{1} \times B^{1}=\partial X \times B^{2}$. Moreover, we obtain a handle decomposition of $S^{n} \times B^{1}$ on $\partial X \times B^{2}$ with $k r$-handles, $k(n-r+1)$-handles and two $n$-handles; each handle is of the form (handle of $S^{n}$ on $\left.\partial X \times B^{1}\right) \times B^{1}$. The attaching tube of each $r$-handle may be taken as contained in $\partial X \times-1 \times 0 \subset \partial X \times \partial\left(B^{1} \times B^{1}\right)$, and the attaching tube of each $(n-r+1)$-handle as contained in $\partial X \times 1 \times 0$.

If $P=\operatorname{cl}\left[S^{n+1}-\partial X \times B^{2}\right]$, then by adding two $(n+1)$-handles to $S^{n} \times B^{1}$ we obtain a handle decomposition of $P$ on $\partial X \times B^{2}$.

In these circumstances, we say that $X$ and $\partial X$ are unknotted in $S^{n+1}$. From now on, we shall be concerned with the cases $n=2 q$, and $r=q$ or $q+1$, with $q \geqslant 3$.

If ${ }^{\sim}$ denotes the universal (= infinite cyclic) cover, then $\partial \tilde{P}=\tilde{\partial P}$, and in each dimension $H_{*}(\tilde{P})$ and $H_{*}(\tilde{P}, \partial \tilde{P})$ are finitely-generated $\Lambda$-modules. The following results are immediate consequences of the handle decomposition of $P$ on $\partial P$, taking $r=q$ and $k=m$.

LEMMA 6.1. $(P, \partial P)$ is $(q-1)$-connected.

LEMMA 6.2. $H_{q}(\tilde{P}, \partial \tilde{P}) \cong \bigoplus_{1}^{m} \Lambda$ with basis $\left[\tilde{x}_{1}\right], \ldots,\left[\tilde{x}_{m}\right]$, where $x_{i}$ is the core of the ith $q$-handle, and $H_{q+1}(\tilde{P}, \partial \tilde{P})=\bigoplus_{1}^{m} \Lambda$ with basis $\left[\tilde{X}_{1}^{*}\right], \ldots,\left[\tilde{X}_{m}^{*}\right]$, where $X_{i}^{*}$ is the core of the ith $(q+1)$-handle, corresponding to the cocore of $h_{i}^{q-1}$.

Note that $\tilde{x}$ denotes a fixed lift of $x \subset P$ to $\tilde{P}$.

Let $S_{\tilde{p}}: H_{s}(\tilde{P}, \partial \tilde{P}) \times H_{2 q+1-s}(\tilde{P}) \rightarrow \Lambda$ denote the Blanchfield intersection pairing [B]. We shall take $\tilde{x}_{1}, \ldots, \tilde{x}_{m}$ as a basis for $C_{q}(\tilde{P}, \partial \tilde{P})$, and $\tilde{X}_{1}^{*}, \ldots, \tilde{X}_{m}^{*}$ as a basis for $C_{q+1}(\tilde{P}, \partial \tilde{P})$, and let $S_{\tilde{p}}$ denote the intersection pairing at the chain level, $C_{s}(\tilde{P}, \partial \tilde{P})$ $\times C_{2 q+1-s}(\tilde{P}) \rightarrow \Lambda$. As in [B], $S_{\tilde{P}}$ has the following properties, where $x, y \in$ $H_{s}(\tilde{P}, \partial \tilde{P}), v, w \in H_{2 q+1-s}(\tilde{P}), u \in H_{s}(\tilde{P}), \alpha, \beta \in \Lambda$, and $i_{*}: H_{*}(\tilde{P}) \rightarrow H_{*}(\tilde{P}, \partial \tilde{P})$ is the usual map. 
(1) $S(x+y, v)=S(x, v)+S(y, v)$.

(2) $S(x, v+w)=S(x, v)+S(x, w)$.

(3) $S(\alpha x, \bar{\beta} v)=\alpha \beta S(x, v)$.

(4) $S\left(i_{*} u, v\right)=(-1)^{s(2 q-s+1)} \overline{S\left(i_{*} v, u\right)}=\overline{S\left(i_{*} v, u\right)}$.

Let $S^{2 q}$ and $S^{2 q+1}$ be oriented so that $t$ in $(t:)=\pi_{1}(P)$ corresponds to the negative normal of $X$.

The $q$-ball $x_{i}$ is contained in a $(q+1)$-ball $(1+\varepsilon) x_{i} \times B^{1}$ which meets $X$ transversely in $(1+\varepsilon) x_{i}$ (here $\varepsilon$ is a small positive number), and the boundary of this $(q+1)$-ball is a $q$-sphere $X_{i}$ embedded in $P$. Similarly the $(q+1)$-ball $X_{i}^{*}$ yields a $(q+1)$-sphere $x_{i}^{*}$ embedded in $P$. Their properties are summarised in the following lemma; see [K3] for details. Square brackets denote homology class.

Lemma 6.3. $H_{q}(\tilde{P}) \cong \oplus_{1}^{m} \Lambda$ with basis $\left[\tilde{X}_{1}\right], \ldots,\left[\tilde{X}_{m}\right]$, and $H_{q+1}(\tilde{P}) \cong \oplus_{1}^{m} \Lambda$ with basis $\left[\tilde{x}_{1}^{*}\right], \ldots,\left[\tilde{x}_{m}^{*}\right]$. For $1 \leqslant i \leqslant m, 1 \leqslant j \leqslant m$ :

$$
\begin{gathered}
i_{*}\left[\tilde{X}_{i}\right]=(1-t)\left[\tilde{x}_{i}\right], \quad i_{*}\left[\tilde{x}_{i}^{*}\right]=\left(1-t^{-1}\right)\left[\tilde{X}_{i}^{*}\right] ; \\
S_{\tilde{p}}\left(\tilde{x}_{i}, \tilde{x}_{j}^{*}\right)=\delta_{i j}, \quad S_{\tilde{p}}\left(\tilde{X}_{i}^{*}, \tilde{X}_{j}\right)=\delta_{i j} \cdot \square
\end{gathered}
$$

Recall that we started with a handle decomposition of $S^{2 q}$, with a single 0 -handle, $m$ trivial pairs of $(q-1)$ and $q$-handles, and a $2 q$-handle. Now augment this handle decomposition by adding $n$ trivial pairs of $q$ and $(q+1)$-handles, and set $Y=B^{2 q}$ $\cup \cup_{i=1}^{m} h_{i}^{q-1} \cup \cup_{j=m+1}^{m+n} h_{j}^{q}$, where the new handle pairs are $\left(h_{j}^{q}, h_{j}^{q+1}\right), m+1 \leqslant j$ $\leqslant m+n$. The construction above carries through with $Y$ in place of $X$, and we set $Q=\operatorname{cl}\left[S^{2 q+1}-\partial Y \times B^{2}\right] . Y$ and $\partial Y$ are unknotted in $S^{2 q+1}$. Note that $Q$ has a handle decomposition on $\partial Y \times B^{2}$ consisting of $q$ and $(q+1)$-handles, and two $2 q$ and $(2 q+1)$-handles. The attaching tubes of the first $m q$-handles, with cores corresponding to the cores of $h_{i}^{q}(1 \leqslant i \leqslant m)$, and those of the final $n(q+1)$-handles, with cores corresponding to the cores of $h_{i}^{q+1}(m+1 \leqslant i \leqslant m+n)$, are contained in $\partial Y \times-1 \times 0 \subset \partial Y \times \partial\left(B^{1} \times B^{1}\right)=\partial Q$, whilst the attaching tubes of the final $n$ $q$-handles, with cores corresponding to the cocores of $h_{i}^{q}(m+1 \leqslant i \leqslant m+n)$, and those of the first $m(q+1)$-handles, with cores corresponding to the cocores of $h_{i}^{q-1}$ $(1 \leqslant i \leqslant m)$, are contained in $\partial Y \times 1 \times 0 \subset \partial Q$.

LEMMA 6.4. $(Q, \partial Q)$ is $(q-1)$-connected.

LEMMA 6.5. $H_{q}(\tilde{Q}, \partial \tilde{Q}) \cong \bigoplus_{1}^{m+n} \Lambda$ with basis $\left[\tilde{y}_{1}\right], \ldots,\left[\tilde{y}_{m+n}\right]$ where for $1 \leqslant i \leqslant m$, $\tilde{y}_{i}=\tilde{x}_{i}$, and for $m \leqslant i \leqslant m+n, y_{i}$ corresponds to the cocore of $h_{i}^{q}$.

$H_{q+1}(\tilde{Q}, \partial \tilde{Q}) \cong \oplus_{1}^{m+n} \Lambda$ with basis $\left[\tilde{Y}_{1}^{*}\right], \ldots,\left[\tilde{Y}_{m+n}^{*}\right]$ where for $1 \leqslant i \leqslant m, \tilde{Y}_{i}^{*}=\tilde{X}_{i}^{*}$, and for $m<i \leqslant m+n, Y_{i}^{*}$ corresponds to the core of $h_{i}^{q+1}$.

Note that here we are regarding $Q$ as a subset of $P$ : we could equally well have described $y_{i}$ as corresponding to the core of $h_{i}^{q}(1 \leqslant i \leqslant m)$, and $Y_{i}^{*}$ as corresponding to the cocore of $h_{i}^{q-1}(1 \leqslant i \leqslant m)$.

LEMMA 6.6. $H_{q}(\tilde{Q}) \cong \oplus_{1}^{m+n} \Lambda$ with basis $\left[\tilde{Y}_{1}\right], \ldots,\left[\tilde{Y}_{m+n}\right]$ where $i_{*}\left[\tilde{Y}_{i}\right]=$ $(1-t)\left[\tilde{y}_{i}\right] ; \quad H_{q+1}(\tilde{Q}) \cong \oplus_{1}^{m+n}$ with basis $\left[\tilde{y}_{1}^{*}\right], \ldots,\left[\tilde{y}_{m+n}^{*}\right]$ where $i_{*}\left[\tilde{y}_{i}^{*}\right]=$ $\left(1-t^{-1}\right)\left[\tilde{Y}_{i}^{*}\right]$; and $S_{\tilde{Q}}\left(\tilde{y}_{i}, \tilde{y}_{j}^{*}\right)=\delta_{i j}=S_{\tilde{Q}}\left(\tilde{Y}_{i}^{*}, \tilde{Y}_{j}\right)$. 
LEMMA 6.7. Let $\left[\underline{\tilde{y}}_{i}\right]=\left[\tilde{y}_{i}\right]$ for $m<i \leqslant m+n,\left[\underline{\tilde{Y}}_{i}^{*}\right]=\left[\tilde{Y}_{i}^{*}\right]$ for $1 \leqslant i \leqslant m$, and

$$
\begin{aligned}
& {\left[\tilde{y}_{i}\right]=\sum_{j=1}^{m} u_{i j}\left[\tilde{y}_{j}\right]+\sum_{j=1}^{n} v_{i j}\left[\tilde{y}_{m+j}\right], \quad 1 \leqslant i \leqslant m ;} \\
& {\left[\underline{\tilde{Y}}_{m+i}^{*}\right]=\sum_{j=1}^{m} \alpha_{i j}\left[\tilde{Y}_{j}^{*}\right]+\sum_{j=1}^{n} \beta_{i j}\left[\tilde{Y}_{m+j}^{*}\right], \quad 1 \leqslant i \leqslant n ;}
\end{aligned}
$$

so that $\left[\tilde{y}_{i}\right],\left[\tilde{y}_{i}^{*}\right],\left[\underline{\tilde{Y}}_{i}\right],\left[\underline{\tilde{Y}}_{i}^{*}\right]$ are related in the same way as $\left[\tilde{y}_{i}\right],\left[\tilde{y}_{i}^{*}\right],\left[\tilde{Y}_{i}\right],\left[\tilde{Y}_{i}^{*}\right]$. Then $U=I_{m}, \tilde{\beta}=\bar{I}_{n}, \alpha^{*}+V=0$.

Proof. Let $C=\left(\begin{array}{ll}U & V \\ 0 & I_{n}\end{array}\right), \gamma=\left(\begin{array}{cc}I_{m} & 0 \\ \alpha & \beta\end{array}\right)$; so that

$$
\left[\tilde{y}_{i}\right]=\sum_{j=1}^{m+n} c_{i j}\left[\tilde{y}_{i}\right] \text { and }\left[\tilde{\tilde{Y}}_{i}^{*}\right]=\sum_{j=1}^{m+n} \gamma_{i j}\left[\tilde{Y}_{j}^{*}\right] \text {. }
$$

Then

$$
\begin{aligned}
\delta_{i j} & =S_{\tilde{Q}}\left(\left[\tilde{y}_{i}\right],\left[\tilde{y}_{j}^{*}\right]\right) \\
& =S_{\tilde{Q}}\left(\sum_{k=1}^{m+n} c_{i k}\left[\tilde{y}_{k}\right], \sum_{l=1}^{m+n} \gamma_{j l}\left[\tilde{y}_{l}^{*}\right]\right) \\
& =\sum_{k=1}^{m+n} \sum_{l=1}^{m+n} c_{i k} \bar{\gamma}_{j l} \delta_{k l}=\sum_{k=1}^{m+n} c_{i k} \bar{\gamma}_{j k}=\left(C \gamma^{*}\right)_{i j} .
\end{aligned}
$$

Therefore $C \gamma^{*}=I$, and so

$$
I=\left(\begin{array}{cc}
U & V \\
0 & I_{n}
\end{array}\right)\left(\begin{array}{cc}
I_{m} & \alpha^{*} \\
0 & \beta^{*}
\end{array}\right)=\left(\begin{array}{cc}
U & U \alpha^{*}+V \beta^{*} \\
0 & \beta^{*}
\end{array}\right),
$$

whence $U=I_{m}, \beta=I_{n}, \alpha^{*}+V=0$.

Now suppose that we start with another handle decomposition of $S^{2 q}$, involving $B^{2 q}$, trivial pairs $\left(h_{i}^{q-1}, \underline{h}_{i}^{q}\right)$ for $1 \leqslant i \leqslant m$, trivial pairs $\left(h_{i}^{q}, \underline{h}_{i}^{q+1}\right)$ for $m<i \leqslant m+n$, and a $2 q$-handle. Let $y_{i}$, etc., be defined in the same way as in $y_{i}$. Thus $y_{i}=y_{i}$ for $m<i \leqslant m+n$. Thus we have another handle decomposition of $(Q, \bar{\partial} Q)$, and another set of bases for $H_{*}(\tilde{Q}, \partial \tilde{Q})$ and $H_{*}(\tilde{Q})$.

LEMMA 6.8. Let $\left[y_{i}\right]$ be related to $\left[y_{i}\right]$ as in Lemma 6.7. Then there is an ambient isotopy $F$ of $S^{2 q+1}$ such that $F_{0}=\mathrm{id}, F_{1}(Y)=Y$, and $F_{1}\left(y_{i}\right)=y_{i}$ for $1 \leqslant i \leqslant m+n$.

Proof. As we remarked above, $\underline{y}_{i}=y_{i}$ for $m<i \leqslant m+n$. Let $Z=B^{2 q} \cup$ $\cup_{i=m+1}^{m+n} h_{i}^{q}$, with $R$ denoting the exterior of $Z$. Then for $1 \leqslant i \leqslant m, y_{i}$ and $y_{i}$ are $q$-balls embedded in $R$ such that $y_{i} \cap \partial R=\partial y_{i} \cap \partial R$ is a $(q-1)$-ball contained within $\partial Z \times-1 \times 0 \subset \partial Z \times \partial\left(B^{1} \times B^{1}\right)=\partial R$. There is an ambient isotopy of $\partial Z \times-1 \times 0$ taking $\partial y_{i} \cap \partial R$ onto $\partial y_{i} \cap \partial R$, and this extends to an ambient isotopy of $\partial R$, and then to an ambient isotopy of $R$ taking $y_{i}$ onto $y_{i}$, keeping $\partial y_{i} \cap \partial R$ within $\partial Z \times-1 \times 0$. Since $y_{i}$ is a spine of the $2 q$-ball $h_{i}^{q-1} \cup \bar{h}_{i}^{q}$, we can assume that this isotopy extends to an ambient isotopy of $S^{2 q+1}$ taking $Y$ onto itself. For $m<i \leqslant m+n, y_{i} \subset$ int $Z$, and so we can assume that the isotopy leaves $y_{i}=y_{i}$ fixed. 
COROLlaRy 6.9. For $1 \leqslant i \leqslant m$, let $u_{i}$ be a q-ball properly embedded in $Q$, such that $\left[\tilde{u}_{i}\right]=\sum_{j=1}^{m} a_{i j}\left[\tilde{y}_{j}\right]+\sum_{j=1}^{n} s_{i j}\left[\tilde{y}_{m+j}\right]$. Let $F$ be the isotopy of Lemma 6.8: then $\left[F\left(\tilde{u}_{i}\right)\right]$ $=\sum_{j=1}^{m} a_{i j}\left[\tilde{y}_{j}\right]+\sum_{j=1}^{n}\left(s_{i j}+e_{i j}\right)\left[\tilde{y}_{m+j}\right]$ where $E=A V$ and $V$ is the matrix of Lemma 6.7 .

Proof.

$$
\begin{aligned}
{\left[F\left(\tilde{u}_{i}\right)\right] } & =\sum_{j=1}^{m} a_{i j}\left[F\left(\tilde{y}_{j}\right)\right]+\sum_{j=1}^{n} s_{i j}\left[F\left(\tilde{y}_{m+j}\right)\right] \\
& =\sum_{j=1}^{m} a_{i j}\left[\tilde{y}_{j}\right]+\sum_{j=1}^{n} s_{i j}\left[\tilde{y}_{m+j}\right] \\
& =\sum_{j=1}^{m} a_{i j}\left(\left[\tilde{y}_{j}\right]+\sum_{k=1}^{n} v_{j k}\left[\tilde{y}_{m+k}\right]\right)+\sum_{j=1}^{n} s_{i j}\left[\tilde{y}_{m+j}\right] \\
& =\sum_{j=1}^{m} a_{i j}\left[\tilde{y}_{j}\right]+\sum_{j=1}^{n}\left(s_{i j}+\sum_{k=1}^{m} a_{i k} v_{k j}\right)\left[\tilde{y}_{m+j}\right] .
\end{aligned}
$$

So far we have considered $y_{i}$ as an oriented $q$-ball properly embedded in $Q$; it will now be convenient to regard it as a proper embedding $y_{i}: B^{q} \rightarrow Q$, and to denote its homotopy class rel $\partial$ by $\left(y_{i}\right)$. Of course, $\tilde{y}_{i}$ will denote a lift $\tilde{y}_{i}: B^{q} \rightarrow \tilde{Q}$, and $\left[\tilde{y}_{i}\right]$ its homology class in $H_{q}(\tilde{Q}, \partial \tilde{Q})$. Similar remarks apply to $Y_{i}$, etc. Recall that $\zeta$ represents the nonzero element of $\pi_{q+1}\left(S^{q}\right)$, and let $\xi=i_{*}(\zeta)$ where $i_{*}: \pi_{q+1}\left(S^{q}\right) \rightarrow$ $\pi_{q+1}\left(B^{q}, \partial B^{q}\right)$ is the canonical isomorphism.

Let $M=\partial Y$, so that $\partial Q \cong M \times S^{1}$. The next result gives a necessary and sufficient condition for $m+n$ singular $(q+1)$-balls $\underline{Y}_{i}^{*}: B^{q+1} \rightarrow Q$ to be homotopic to the cores of $(q+1)$-handles in a handle decomposition of $(Q, \partial Q)$. For the rest of this section, we assume that $q \geqslant 5$.

LEMMA 6.10. Let $\underline{Y}_{i}^{*}=Y_{i}^{*}, 1 \leqslant i \leqslant m$, and

$$
\left(\underline{\tilde{Y}}_{m+i}^{*}\right)=\left(\tilde{Y}_{m+i}^{*}\right)+\sum_{j=1}^{n} \alpha_{i j}\left(\tilde{y}_{m+j}\right) \circ \xi, \quad 1 \leqslant i \leqslant n,
$$

and $i_{*}\left(\tilde{y}_{i}^{*}\right)=\left(1-t^{-1}\right)\left(\underline{Y}_{i}^{*}\right)$ for $1 \leqslant i \leqslant m+n$. Assume that $\partial \underline{Y}_{i}^{*}: \partial B^{q} \rightarrow M \times * \subset$ $M \times S^{1^{-}}=\partial Q$ is an embedding for each $i$, as for $\partial Y_{i}^{*}$, for some point $* \in S^{1}$. Then $\cup_{i=1}^{m+n} \underline{Y}_{i}^{*}$ can be homotoped (rel $\partial$ ) to be a set of cores of $(q+1)$-handles in a handle decomposition of $(Q, \partial Q)$ if and only if $\alpha=t \alpha^{*}$.

Proof. By [K1] there is an $(m+n) \times(m+n)$ matrix $\mathbb{Q}$ over $\Gamma$ which is the obstruction to homotoping $\cup_{i=1}^{m+n} \underline{Y}_{i}^{*}$ to an embedding (rel $\left.\partial\right)$, and since $i_{*}\left(\tilde{y}_{i}^{*}\right)=$ $\left(1-t^{-1}\right)\left(\underline{Y}_{i}^{*}\right)$, it follows at once from the definition of $\mathscr{Q}$ that

$$
T_{\tilde{Q}}\left(\left(\underline{\tilde{Y}}_{i}^{*}\right),\left(\underline{\tilde{y}}_{j}^{*}\right)\right)=(1-t) \mathbb{Q}_{i j}
$$

Thus $\cup_{i=1}^{m+n} \underline{Y}_{i}^{*}$ can be homotoped (rel $\partial$ ) to an embedding if and only if $T_{\tilde{Q}}\left(\left(\underline{\tilde{Y}}_{i}^{*}\right),\left(\underline{\tilde{y}}_{j}^{*}\right)\right)=0$ for all $i, j$. 
Note that the following intersections hold for all $i, j$.

$$
\left\{\begin{array}{l}
T_{\tilde{Q}}\left(\left(\tilde{Y}_{i}^{*}\right),\left(\tilde{y}_{j}^{*}\right)\right)=0, \\
T_{\tilde{Q}}\left(\left(\tilde{Y}_{i}^{*}\right),\left(\tilde{Y}_{j}^{*}\right) \circ \zeta\right)=\delta_{i j}, \\
T_{\tilde{Q}}\left(\left(\tilde{y}_{i}\right) \circ \xi,\left(\tilde{y}_{j}^{*}\right)\right)=\delta_{i j}, \\
T_{\tilde{Q}}\left(\left(\tilde{y}_{i}\right) \circ \xi,\left(\tilde{Y}_{j}\right) \circ \zeta\right)=0 .
\end{array}\right.
$$

The middle two identities follow from the corresponding equations for $S_{\tilde{Q}}($, ), and the last identity is true from dimensional considerations. Thus only the first identity is of independent interest.

Moreover, for $1 \leqslant i \leqslant n$,

$$
\begin{aligned}
i_{*}\left(\tilde{y}_{m+i}^{*}\right) & =\left(1-t^{-1}\right)\left(\underline{Y}_{m+i}^{*}\right) \\
& =\left(1-t^{-1}\right)\left(\tilde{Y}_{m+i}^{*}\right)+\left(1-t^{-1}\right) \sum_{j=1}^{n} \alpha_{i j}\left(\tilde{y}_{m+j}\right) \circ \xi \\
& =i_{*}\left(\tilde{y}_{m+i}^{*}\right)-t^{-1} \sum_{j=1}^{n} \alpha_{i j} i_{*}\left(\tilde{Y}_{m+j}\right) \circ \zeta
\end{aligned}
$$

and so $\left(\tilde{y}_{m+i}^{*}\right)=\left(\tilde{y}_{m+i}^{*}\right)-t^{-1} \sum_{j=1}^{n} \alpha_{i j}\left(\tilde{Y}_{m+j}\right) \circ \zeta$.

For $1 \leqslant i \leqslant m,\left(\tilde{y}_{i}^{*}\right)=\left(\tilde{y}_{i}^{*}\right)$.

Consider $1 \leqslant i \leqslant n, 1 \leqslant k \leqslant m$.

$$
T_{\tilde{Q}}\left(\left(\underline{\tilde{Y}}_{m+i}^{*}\right),\left(\underline{\tilde{y}}_{k}^{*}\right)\right)=T_{\tilde{Q}}\left(\left(\tilde{Y}_{m+i}^{*}\right)+\sum_{j=1}^{n} \alpha_{i j}\left(\tilde{y}_{m+j}\right) \circ \xi,\left(\tilde{y}_{k}^{*}\right)\right)=0
$$

using the intersection properties above. Similarly, $T_{\tilde{Q}}\left(\left(\underline{\tilde{Y}}_{i}^{*}\right),\left(\tilde{y}_{k}^{*}\right)\right)=0$ for $1 \leqslant i \leqslant m$, $1 \leqslant k \leqslant m$, and $T_{\tilde{Q}}\left(\left(\underline{\tilde{Y}}_{i}^{*}\right),\left(\tilde{y}_{m+k}^{*}\right)\right)=0$ for $1 \leqslant i \leqslant m, 1 \leqslant k \leqslant n$. Thus the condition $T_{\tilde{Q}}\left(\left(\underline{\tilde{Y}}_{i}^{*}\right),\left(\underline{\tilde{y}}_{j}^{*}\right)\right)=0$ for all $\bar{i}, j$, is equivalent to the condition $T_{\tilde{Q}}\left(\left(\underline{\tilde{Y}}_{m+i}^{*}\right),\left(\underline{\tilde{y}}_{m+j}^{*}\right)\right)=0$ for $1 \leqslant i \leqslant n, 1 \leqslant j \leqslant n$. But

$$
\begin{aligned}
T_{\tilde{Q}}\left(\left(\underline{\tilde{Y}}_{m+i}^{*}\right)\right. & \left.,\left(\underline{\tilde{y}}_{m+j}^{*}\right)\right) \\
& =T_{\tilde{Q}}\left(\left(\tilde{Y}_{m+i}^{*}\right)+\sum_{k=1}^{n} \alpha_{i k}\left(\tilde{y}_{m+k}\right) \circ \xi,\left(\tilde{y}_{m+j}^{*}\right)-t^{-1} \sum_{l=1}^{n} \alpha_{j l}\left(\tilde{Y}_{m+l}\right) \circ \zeta\right) \\
& =\sum_{k=1}^{n} \alpha_{i k} T_{\tilde{Q}}\left(\left(\tilde{y}_{m+k}\right) \circ \xi,\left(\tilde{y}_{m+j}^{*}\right)\right)-t \sum_{l=1}^{n} \bar{\alpha}_{j l} T_{Q}\left(\left(\tilde{Y}_{m+i}^{*}\right),\left(\tilde{Y}_{m+l}\right) \circ \zeta\right) \\
& =\sum_{k=1}^{n} \alpha_{i k} \delta_{k j}-t \sum_{l=1}^{n} \bar{\alpha}_{j l} \delta_{i l} \\
& =\alpha_{i j}-t \bar{\alpha}_{j i} .
\end{aligned}
$$

Thus $\cup_{i=1}^{m+n} \underline{Y}_{i}^{*}$ can be homotoped (rel $\partial$ ) to an embedding if and only if $\alpha=t \alpha^{*}$. In fact, since $\cup_{i=1}^{m} \underline{Y}_{i}^{*}=\cup_{i=1}^{m} Y_{i}^{*}$ is already embedded, the homotopy can be chosen to keep this fixed (compare [K1]).

Assume that the condition is satisfied, and that $\cup_{i=1}^{m+n} \underline{Y}_{i}^{*}$ is embedded. Since $\left[\underline{\tilde{Y}}_{i}^{*}\right]=\left[Y_{i}^{*}\right]$ for each $i$, we have $S_{Q}\left(\underline{\tilde{Y}}_{i}^{*}, \tilde{Y}_{j}\right)=\delta_{i j}$ for each $i, j$. By the Whitney lemma 
(or by adapting the arguments of [K1]), we can isotop $\cup_{i=1}^{m+n} \underline{Y}_{i}^{*}$ to be disjoint from $\cup_{i=1}^{m+n} \tilde{y}_{i}$. Let $N$ be a regular neighbourhood in $Q$ of $\partial Q \cup \cup_{i=1}^{m+n} \tilde{y}_{i} \cup \cup_{i=1}^{m+n} \underline{Y}_{i}^{*}$. Because $(Q, \partial Q)$ is $(q-1)$-connected, and $\cup_{i=1}^{m+n} \tilde{y}_{i}, \cup_{i=1}^{m+n} \underline{Y}_{i}^{*}$ are bases of $H_{q}(\tilde{Q}, \partial \tilde{Q}), H_{q+1}(\tilde{Q}, \partial \tilde{Q})$ respectively represented by balls properly and disjointly embedded in $Q$, it follows that $\overline{Q-N}$ is a ball, whence the desired conclusion.

LEMMA 6.11. Let $\underline{Y}_{i}$ be related to $Y_{i}$ as in Lemma 6.10, and assume that $\cup_{i=1}^{m+n} \underline{Y}_{i}$ is embedded. Then there is an ambient isotopy $F$ of $S^{2 q+1}$ such that $F_{0}=\mathrm{id}, F_{1}(Y)=Y$, and $F_{1}\left(Y_{i}^{*}\right)=\underline{Y}_{i}^{*}$ for $1 \leqslant i \leqslant m+n$.

Proof. The proof is similar to that of Lemma 6.8.

LEMMA 6.12. Let

$$
\left(\tilde{V}_{m+i}^{*}\right)=\sum_{j=1}^{m} c_{i j}\left(\tilde{Y}_{j}^{*}\right)+\sum_{j=1}^{n} b_{i j}\left(\tilde{Y}_{m+j}^{*}\right)+\sum_{j=1}^{m} d_{i j}\left(\tilde{y}_{j}\right) \circ \xi+\sum_{j=1}^{n} e_{i j}\left(\tilde{y}_{m+j}\right) \circ \xi
$$

for $1 \leqslant i \leqslant n$, and let $F$ be the isotopy of the previous lemma. Then

$$
\begin{aligned}
\left(F\left(\tilde{V}_{m+i}^{*}\right)\right)= & \sum_{j=1}^{m} c_{i j}\left(\tilde{Y}_{j}^{*}\right)+\sum_{j=1}^{n} b_{i j}\left(\tilde{Y}_{m+j}^{*}\right)+\sum_{j=1}^{m} d_{i j}\left(\tilde{y}_{j}\right) \circ \xi \\
& +\sum_{j=1}^{n}\left\{e_{i j}+\sum_{k=1}^{n} b_{i k} \alpha_{k j}\right\}\left(\tilde{y}_{m+j}\right) \circ \xi .
\end{aligned}
$$

ProOF.

$$
\begin{aligned}
\left(F\left(\tilde{V}_{m+i}^{*}\right)\right)= & \sum_{j=1}^{m} c_{i j}\left(F\left(\tilde{Y}_{j}^{*}\right)\right)+\sum_{j=1}^{n} b_{i j}\left(F\left(\tilde{Y}_{m+j}^{*}\right)\right) \\
& +\sum_{j=1}^{m} d_{i j}\left(F\left(\tilde{y}_{j}\right)\right) \circ \xi+\sum_{j=1}^{n} e_{i j}\left(F\left(\tilde{y}_{m+j}\right)\right) \circ \xi \\
= & \sum_{j=1}^{m} c_{i j}\left(\tilde{Y}_{j}^{*}\right)+\sum_{j=1}^{n} b_{i j}\left\{\left(\tilde{Y}_{m+j}^{*}\right)+\sum_{k=1}^{n} \alpha_{j k}\left(\tilde{y}_{m+k}\right) \circ \xi\right\} \\
& +\sum_{j=1}^{m} d_{i j}\left(\tilde{y}_{j}\right) \circ \xi+\sum_{j=1}^{n} e_{i j}\left(\tilde{y}_{m+j}\right) \circ \xi \\
= & \sum_{j=1}^{m} c_{i j}\left(\tilde{Y}_{j}^{*}\right)+\sum_{j=1}^{n} b_{i j}\left(\tilde{Y}_{m+j}^{*}\right)+\sum_{j=1}^{m} d_{i j}\left(\tilde{y}_{j}\right) \circ \xi \\
& +\sum_{j=1}^{n}\left\{e_{i j}+\sum_{k=1}^{n} b_{i k} \alpha_{k j}\right\}\left(\overline{\tilde{y}}_{m+j}\right) \circ \xi .
\end{aligned}
$$

Consider now possibly singular $q$-balls $v_{i}, 1 \leqslant i \leqslant m+n$, and $(q+1)$-balls $V_{i}^{*}$, $1 \leqslant i \leqslant m+n$, properly contained in $Q$. Furthermore, if $\partial Q=M \times S^{1}$, we assume that $\cup_{i=1}^{n} \partial v_{i} \cup \cup_{j=1}^{n} \partial V_{m+j}^{*}$ is embedded in $M \times-1$ and that $\cup_{i=1}^{n} \partial v_{m+i} \cup$ $\cup_{j=1}^{m} \partial V_{j}^{*}$ is embedded in $M \times 1$. We shall analyse the conditions under which $\cup_{i=1}^{m+n} v_{i} \cup \cup_{j=1}^{m+n} V_{j}^{*}$ can be homotoped (rel $\partial$ ) to be properly embedded in $Q$. 
Assume that

$$
\begin{gathered}
{\left[\tilde{v}_{i}\right]=\sum_{j=1}^{m} a_{i j}\left[\tilde{y}_{j}\right]+\sum_{j=1}^{n} s_{i j}\left[\tilde{y}_{m+j}\right], \quad 1 \leqslant i \leqslant m ;} \\
{\left[\tilde{v}_{m+i}\right]=\sum_{j=1}^{m} p_{i j}\left[\tilde{y}_{j}\right]+\sum_{j=1}^{n} q_{i j}\left[\tilde{y}_{m+j}\right], \quad 1 \leqslant i \leqslant n ;} \\
\left(\tilde{V}_{i}^{*}\right)=\sum_{j=1}^{m} m_{i j}\left(\tilde{Y}_{j}^{*}\right)+\sum_{j=1}^{n} n_{i j}\left(\tilde{Y}_{m+j}^{*}\right)+\sum_{j=1}^{m} f_{i j}\left(\tilde{y}_{j}\right) \circ \xi \\
+\sum_{j=1}^{n} g_{i j}\left(\tilde{y}_{m+j}\right) \circ \xi, \quad 1 \leqslant i \leqslant m ; \\
\left(\tilde{V}_{m+i}^{*}\right)=\sum_{j=1}^{m} c_{i j}\left(\tilde{Y}_{j}^{*}\right)+\sum_{j=1}^{n} b_{i j}\left(\tilde{Y}_{m+j}^{*}\right)+\sum_{j=1}^{m} d_{i j}\left(\tilde{y}_{j}\right) \circ \xi \\
+\sum_{j=1}^{n} e_{i j}\left(\tilde{y}_{m+j}\right) \circ \xi, \quad 1 \leqslant i \leqslant n .
\end{gathered}
$$

Define $\left(\tilde{V}_{i}\right),\left(\tilde{v}_{i}^{*}\right)$ by the equations $i_{*}\left(\tilde{V}_{i}\right)=(1-t)\left(\tilde{v}_{i}\right), i_{*}\left(\tilde{v}_{i}^{*}\right)=\left(1-t^{-1}\right)\left(\tilde{V}_{i}^{*}\right)$.

LEMMA 6.13.

$$
\begin{aligned}
\left(\tilde{v}_{i}^{*}\right)= & \sum_{j=1}^{m} m_{i j}\left(\tilde{y}_{j}^{*}\right)+\sum_{j=1}^{n} n_{i j}\left(\tilde{y}_{m+j}^{*}\right)-t^{-1} \sum_{j=1}^{m} f_{i j}\left(\tilde{Y}_{j}\right) \circ \zeta \\
& -t^{-1} \sum_{k=1}^{n} g_{i j}\left(\tilde{Y}_{m+j}\right) \circ \zeta, \quad 1 \leqslant i \leqslant m ; \\
\left(\tilde{v}_{m+i}^{*}\right)= & \sum_{j=1}^{m} c_{i j}\left(\tilde{y}_{j}^{*}\right)+\sum_{j=1}^{n} b_{i j}\left(\tilde{y}_{m+j}^{*}\right)-t^{-1} \sum_{j=1}^{m} d_{i j}\left(\tilde{Y}_{j}\right) \circ \zeta \\
& -t^{-1} \sum_{j=1}^{n} e_{i j}\left(\tilde{Y}_{m+j}\right) \circ \zeta, \quad 1 \leqslant i \leqslant n .
\end{aligned}
$$

Proof. For $1 \leqslant i \leqslant m$,

$$
\begin{aligned}
i_{*}\left(\tilde{v}_{i}^{*}\right)= & \left(1-t^{-1}\right)\left(\tilde{V}_{i}^{*}\right) \\
= & \sum_{j=1}^{m} m_{i j}\left(1-t^{-1}\right)\left(\tilde{Y}_{j}^{*}\right)+\sum_{j=1}^{n} n_{i j}\left(1-t^{-1}\right)\left(\tilde{Y}_{m+j}^{*}\right) \\
& +\sum_{j=1}^{m} f_{i j}\left(1-t^{-1}\right)\left(\tilde{y}_{j}\right) \circ \xi+\sum_{j=1}^{n} g_{i j}\left(1-t^{-1}\right)\left(\tilde{y}_{m+j}\right) \circ \xi \\
= & i_{*} \sum_{j=1}^{m} m_{i j}\left(\tilde{y}_{j}^{*}\right)+i_{*} \sum_{j=1}^{n} n_{i j}\left(\tilde{y}_{m+j}^{*}\right) \\
& -i_{*} t^{-1} \sum_{j=1}^{m} f_{i j}\left(\tilde{Y}_{j}\right) \circ \zeta-i_{*} t^{-1} \sum_{j=1}^{n} g_{i j}\left(\tilde{Y}_{m+j}\right) \circ \zeta,
\end{aligned}
$$


from which the result follows as $i_{*}$ is a monomorphism (because the map $\pi_{q+1}(\partial \tilde{Q})$ $\rightarrow \pi_{q+1}(\tilde{Q})$ induced by inclusion is the zero map, from the handle decomposition of $Q)$. The proof for $\left(v_{m+i}^{*}\right)$ is similar.

Of course, the expression for $\left[\tilde{V}_{i}\right]$ in terms of the $\left[\tilde{Y}_{j}\right]$ is the same as that for $\left[\tilde{v}_{i}\right]$ in terms of the $\left[\tilde{y}_{j}\right]$.

LEMMA 6.14. $S_{\tilde{Q}}\left(\left[\tilde{V}_{i}^{*}\right],\left[\tilde{V}_{k}\right]\right)=\delta_{i k}$ for all $i, k \Leftrightarrow\left(\begin{array}{cc}M & N \\ C & B\end{array}\right)\left(\begin{array}{c}A^{*} \\ S^{*}\end{array} Q^{*}\right)=\left(\begin{array}{cc}I_{m} & 0 \\ 0 & I_{n}\end{array}\right)$.

ProOF. For $1 \leqslant i \leqslant m, 1 \leqslant k \leqslant m$, we have

$$
\begin{aligned}
S_{\tilde{Q}}\left(\left[\tilde{V}_{i}^{*}\right],\left[\tilde{V}_{k}\right]\right) & =S_{\tilde{Q}}\left(\sum_{j=1}^{m} m_{i j}\left[\tilde{Y}_{j}^{*}\right]+\sum_{j=1}^{n} n_{i j}\left[\tilde{Y}_{m+j}^{*}\right], \sum_{l=1}^{m} a_{k l}\left[\tilde{Y}_{l}\right]+\sum_{l=1}^{n} s_{k l}\left[\tilde{Y}_{m+l}\right]\right) \\
& =\sum_{j=1}^{m} \sum_{l=1}^{m} m_{i j} \bar{a}_{k l} \delta_{j l}+\sum_{j=1}^{n} \sum_{l=1}^{n} n_{i j} \bar{s}_{k l} \delta_{j l} \\
& =\left(M A^{*}+N S^{*}\right)_{i k},
\end{aligned}
$$

and so for $i, k$ in this range,

$$
S_{Q}\left(\left[\tilde{V}_{i}^{*}\right],\left[\tilde{V}_{k}\right]\right)=\delta_{i k} \Leftrightarrow M A^{*}+N S^{*}=I_{m} .
$$

The other identities are proved in a similar manner.

Define $a_{i j}^{*}=\bar{a}_{j i}$; thus if $A=\left(a_{i j}\right)$, then $A^{*}=\left(a_{i j}^{*}\right)$. Assume now that

$$
\left(\begin{array}{ll}
M & N \\
C & B
\end{array}\right)\left(\begin{array}{ll}
A^{*} & P^{*} \\
S^{*} & Q^{*}
\end{array}\right)=\left(\begin{array}{ll}
I_{m} & 0 \\
0 & I_{n}
\end{array}\right),
$$

and that

$$
\begin{aligned}
\left(\tilde{Y}_{i}^{*}\right)= & \sum_{j=1}^{m} a_{i j}^{*}\left(\tilde{V}_{j}^{*}\right)+\sum_{j=1}^{n} p_{i j}^{*}\left(\tilde{V}_{m+j}^{*}\right)-t \sum_{j=1}^{m} h_{i j}\left(\tilde{v}_{j}\right) \circ \xi \\
& -t \sum_{j=1}^{n} k_{i j}\left(\tilde{v}_{m+j}\right) \circ \xi, \quad 1 \leqslant i \leqslant m ; \\
\left(\tilde{Y}_{m+i}^{*}\right)= & \sum_{j=1}^{m} s_{i j}^{*}\left(\tilde{V}_{j}^{*}\right)+\sum_{j=1}^{n} q_{i j}^{*}\left(\tilde{V}_{m+j}^{*}\right)-t \sum_{j=1}^{m} l_{i j}\left(\tilde{v}_{j}\right) \circ \xi \\
& -t \sum_{j=1}^{n} r_{i j}\left(\tilde{v}_{m+j}\right) \circ \xi, \quad 1 \leqslant i \leqslant n ;
\end{aligned}
$$

for some matrices $H, K, L, R$ over $\Gamma$.

LEMMA 6.15.

$$
\begin{aligned}
T_{\tilde{Q}}\left(\left(\tilde{V}_{i}^{*}\right),\left(\tilde{v}_{j}^{*}\right)\right) & =0, & T_{\tilde{Q}}\left(\left(\tilde{V}_{i}^{*}\right),\left(\tilde{V}_{j}\right) \circ \zeta\right) & =\delta_{i j}, \\
T_{\tilde{Q}}\left(\left(\tilde{v}_{i}\right) \circ \xi,\left(\tilde{v}_{j}^{*}\right)\right) & =\delta_{i j}, & T_{\tilde{Q}}\left(\left(\tilde{v}_{i}\right) \circ \xi,\left(\tilde{V}_{j}\right) \circ \zeta\right) & =0,
\end{aligned}
$$

for all $i, j$, implies that $\left(\begin{array}{cc}H & K \\ L & R\end{array}\right)=\left(\begin{array}{c}F^{*} D^{*} E^{*} \\ E^{*}\end{array}\right)$.

Note. These identities are similar to (6.1) in the proof of Lemma 6.10, and again only the first identity is of independent interest. 
Proof.

$$
\begin{aligned}
\left(1-t^{-1}\right) f_{i j} & =\left(1-t^{-1}\right) T_{\tilde{Q}}\left(\left(\tilde{V}_{i}^{*}\right),\left(\tilde{y}_{j}^{*}\right)\right) \quad \text { by }(6.1) \text { and }(6.2) \\
& =T_{\tilde{Q}}\left(\left(1-t^{-1}\right)\left(\tilde{V}_{i}^{*}\right),\left(\tilde{y}_{j}^{*}\right)\right)=T_{\tilde{Q}}\left(i_{*}\left(\tilde{v}_{i}^{*}\right),\left(\tilde{y}_{j}^{*}\right)\right) \\
& =\overline{T_{\tilde{Q}}\left(i_{*}\left(\tilde{y}_{j}^{*}\right),\left(\tilde{v}_{i}^{*}\right)\right)}=\overline{T_{\tilde{Q}}\left(\left(1-t^{-1}\right)\left(\tilde{Y}_{j}^{*}\right),\left(\tilde{v}_{i}^{*}\right)\right)} \\
& =(1-t) \overline{T_{\tilde{Q}}\left(\left(\tilde{Y}_{j}^{*}\right),\left(\tilde{v}_{i}^{*}\right)\right)}=(1-t) \overline{\left(-t h_{j i}\right)}=\left(1-t^{-1}\right) \bar{h}_{j i},
\end{aligned}
$$

and so $F=H^{*}$. The remaining identities are proved in a similar manner.

The proof of the following lemma is similar to that of Lemma 6.13, and is omitted.

LEMMA 6.16.

$$
\begin{aligned}
\left(\tilde{y}_{i}^{*}\right)= & \sum_{j=1}^{m} a_{i j}^{*}\left(\tilde{v}_{j}^{*}\right)+\sum_{j=1}^{n} p_{i j}^{*}\left(\tilde{v}_{m+j}^{*}\right)+\sum_{j=1}^{m} h_{i j}\left(\tilde{V}_{j}\right) \circ \zeta \\
& +\sum_{j=1}^{n} k_{i j}\left(\tilde{V}_{m+j}\right) \circ \zeta, \quad 1 \leqslant i \leqslant m ; \\
\left(\tilde{y}_{m+i}^{*}\right)= & \sum_{j=1}^{m} s_{i j}^{*}\left(\tilde{v}_{j}^{*}\right)+\sum_{j=1}^{n} q_{i j}^{*}\left(\tilde{v}_{m+j}^{*}\right)+\sum_{j=1}^{m} l_{i j}\left(\tilde{V}_{j}\right) \circ \zeta \\
& +\sum_{j=1}^{n} r_{i j}\left(\tilde{V}_{m+j}\right) \circ \zeta, \quad 1 \leqslant i \leqslant n .
\end{aligned}
$$

LEMMA 6.17.

$$
\begin{aligned}
& A^{*} F+P^{*} D-t H A-t K P=0 \\
& A^{*} G+P^{*} E-t H S-t K Q=0 \\
& S^{*} F+Q^{*} D-t L A-t R P=0 \\
& S^{*} G+Q^{*} E-t L S-t R Q=0
\end{aligned}
$$

Proof. For $1 \leqslant i \leqslant m$,

$$
\begin{aligned}
\left(\tilde{Y}_{i}^{*}\right)= & \sum_{j=1}^{m} a_{i j}^{*}\left(\tilde{V}_{j}^{*}\right)+\sum_{j=1}^{n} p_{i j}^{*}\left(\tilde{V}_{m+j}^{*}\right)-t \sum_{j=1}^{m} h_{i j}\left(\tilde{v}_{j}\right) \circ \xi-t \sum_{j=1}^{n} k_{i j}\left(\tilde{v}_{m+j}\right) \circ \xi \\
= & \left(\tilde{Y}_{i}^{*}\right)+\sum_{j=1}^{m} a_{i j}^{*}\left\{\sum_{k=1}^{m} f_{j k}\left(\tilde{y}_{k}\right) \circ \xi+\sum_{k=1}^{n} g_{i k}\left(\tilde{y}_{m+k}\right) \circ \xi\right\} \\
& +\sum_{j=1}^{n} p_{i j}^{*}\left\{\sum_{k=1}^{m} d_{j k}\left(\tilde{y}_{k}\right) \circ \xi+\sum_{k=1}^{n} e_{j k}\left(\tilde{y}_{m+k}\right) \circ \xi\right\} \\
& -t \sum_{j=1}^{m} h_{i j}\left\{\sum_{k=1}^{n} a_{j k}\left(\tilde{y}_{k}\right) \circ \xi+\sum_{k=1}^{n} s_{j k}\left(\tilde{y}_{m+k}\right) \circ \xi\right\} \\
& -t \sum_{j=1}^{n} k_{i j}\left\{\sum_{k=1}^{m} p_{j k}\left(\tilde{y}_{k}\right) \circ \xi+\sum_{k=1}^{n} q_{j k}\left(\tilde{y}_{m+k}\right) \circ \xi\right\} \\
= & \left(\tilde{Y}_{i}^{*}\right)+\sum_{k=1}^{m}\left(A^{*} F+P^{*} D-t H A-t K P\right)_{i k}\left(\tilde{y}_{k}\right) \circ \xi \\
& +\sum_{k=1}^{n}\left(A^{*} G+P^{*} E-t H S-t K Q\right)_{i k}\left(\tilde{y}_{m+k}\right) \circ \xi,
\end{aligned}
$$


and this gives the first two equations. The last two are proved similarly by considering $\left(\tilde{Y}_{m+i}^{*}\right)$ for $1 \leqslant i \leqslant n$.

LEMMA 6.18. The converse of Lemma 6.15 is true.

Proof. The first and third equations of Lemma 6.17 yield

$$
\begin{aligned}
& C A^{*} F+C P^{*} D-t C H A-t C K P=0, \\
& B S^{*} F+B Q^{*} D-t B L A-t B R P=0
\end{aligned}
$$

and hence

$$
\left(C A^{*}+B S^{*}\right) F+\left(C P^{*}+B Q^{*}\right) D-t(C H+B L) A-t(C K+B R) P=0 .
$$

But $C A^{*}+B S^{*}=0, C P^{*}+B Q^{*}=I$, and so we have

$$
D-t(C H+B L) A-t(C K+B R) P=0 .
$$

Similarly

$$
\begin{aligned}
E-t(C H+B L) S-t(C K+B R) Q & =0, \\
F-t(M H+N L) A-t(M K+N R) P & =0, \\
G-t(M H+N L) S-t(M K+N R) Q & =0 .
\end{aligned}
$$

Substituting for $H, K, L$ and $R$ gives

(1) $D-t\left(C F^{*}+B G^{*}\right) A-t\left(C D^{*}+B E^{*}\right) P=0$,

(2) $E-t\left(C F^{*}+B G^{*}\right) S-t\left(C D^{*}+B E^{*}\right) Q=0$,

(3) $F-t\left(M F^{*}+N G^{*}\right) A-t\left(M D^{*}+N E^{*}\right) P=0$,

(4) $G-t\left(M F^{*}+N G^{*}\right) S-t\left(M D^{*}+N E^{*}\right) Q=0$.

Adding (1) $C^{*}$ to (2) $B^{*}$ gives

$D C^{*}+E B^{*}-t\left(C F^{*}+B G^{*}\right)\left(A C^{*}+S B^{*}\right)-t\left(C D^{*}+B E^{*}\right)\left(P C^{*}+Q B^{*}\right)=0$,

which since $A C^{*}+S B^{*}=0$ and $P C^{*}+Q B^{*}=I$ gives

(5) $D C^{*}+E B^{*}-t\left(C D^{*}+B E^{*}\right)=0$.

Similarly we can obtain

(6) $D M^{*}+E N^{*}-t\left(C F^{*}+B G^{*}\right)=0$,

(7) $F C^{*}+G B^{*}-t\left(M D^{*}+N E^{*}\right)=0$,

(8) $F M^{*}+G N^{*}-t\left(M F^{*}+N G^{*}\right)=0$.

For $1 \leqslant i \leqslant m, 1 \leqslant k \leqslant m$, we have

$$
\begin{aligned}
T_{\tilde{Q}}\left(\left(\tilde{V}_{i}^{*}\right),\left(\tilde{v}_{k}^{*}\right)\right)=T_{\tilde{Q}}\left(\sum_{j=1}^{m} m_{i j}\left(\tilde{Y}_{j}^{*}\right)+\sum_{j=1}^{n} n_{i j}\left(\tilde{Y}_{m+j}^{*}\right)+\sum_{j=1}^{m} f_{i j}\left(\tilde{y}_{j}\right) \circ \xi\right. \\
\quad+\sum_{j=1}^{n} g_{i j}\left(\tilde{y}_{m+j}\right) \circ \xi, \sum_{j=1}^{m} m_{k j}\left(\tilde{y}_{j}^{*}\right) \\
\left.\quad+\sum_{j=1}^{n} n_{i j}\left(\tilde{y}_{m+j}^{*}\right)-t^{-1} \sum_{j=1}^{m} f_{k j}\left(\tilde{Y}_{j}\right) \circ \zeta-t^{-1} \sum_{j=1}^{n} g_{k j}\left(\tilde{Y}_{m+j}\right) \circ \zeta\right) \\
=-t\left(M F^{*}\right)_{i k}-t\left(N G^{*}\right)_{i k}+\left(F M^{*}\right)_{i k}+\left(G N^{*}\right)_{i k} \\
=0 \text { by }(8) .
\end{aligned}
$$

Similar computations, involving (5), (6), and (7), show that $T_{\tilde{Q}}\left(\left(\tilde{V}_{i}^{*}\right),\left(\tilde{v}_{k}^{*}\right)\right)=0$ for all $i, k$. 
As noted above, the identities involving $\delta_{i j}$ follow at once from the corresponding identities for $S_{\tilde{Q}}($,$) and the remaining identities are true from dimensional$ considerations.

LEMMA 6.19. $\cup_{i=1}^{m+n} v_{i} \cup \cup_{j=1}^{m+n} V_{j}^{*}$ is homotopic (rel d) to a set of cores of handles in a handle decomposition of $(Q, \partial Q)$ if and only if

$$
S_{\tilde{Q}}\left(\left[\tilde{V}_{i}^{*}\right],\left[\tilde{V}_{j}\right]\right)=\delta_{i j} \quad \text { and } \quad T_{\tilde{Q}}\left(\left(\tilde{V}_{i}^{*}\right),\left(\tilde{v}_{j}^{*}\right)\right)=0 \quad \text { for all } i, j .
$$

Proof. The "only if" has already been noted (6.1). Conversely, if these equations are satisfied, then as in the proof of Lemma 6.10, $\cup_{i=1}^{m+n} v_{i} \cup \cup_{j=1}^{m+n} V_{j}^{*}$ can be homotoped (rel $\partial$ ) to an embedded set of balls, and these form the cores of handles in a handle decomposition of $(Q, \partial Q)$.

7. Presentations of knots. We can regard an $n$-knot as an embedding of $S^{n}$ in $S^{n+1} \times I$. A presentation of the knot is one in which $S^{n}=$ handle + collar + handle + collar $+\cdots$, each handle being embedded in a level $S^{n+1} \times x$ and each collar being embedded productwise along the $I$ direction. In the classical case, $n=1$, the 0-handles appear as underpasses and the 1-handles as overpasses. The case $n=2$ is just Fox's method of drawing 2-knots [F]. For further details, see [K3 and K-L].

Each $r$-handle $h^{r}$ in the handle decomposition of $S^{n}$ induces an $(r+1)$-handle $H^{r+1}$ in a handle decomposition of $K$; see Lemma 2.1 of [K3] and [K4] for details. In fact, let $B^{r} \times B^{n-r}$ be the image of $h^{r}$ projected into a higher level $S^{n+1}$, and let $B^{r} \times B^{n-r} \times B^{1}$ be a regular neighbourhood of $B^{r} \times B^{n-r}$ rel $\partial\left(B^{r} \times B^{n-r}\right)$ in $S^{n+1}$. Let $B_{0}^{r}$ be a slightly larger ball containing $B^{r}$ in its interior; then the proofs of [K3, Lemma 2.1 and K-L, Lemma 1] show that the core $C^{r+1}$ of $H^{r+1}$ can be taken as $B_{0}^{r} \times 0 \times B^{1}$ together with a vertical collar of its boundary. The case $n=2, r=1$, is illustrated in Figure 1; the cases $n=1, r=0,1$, are illustrated in [K4].

$x=\frac{3}{4}$

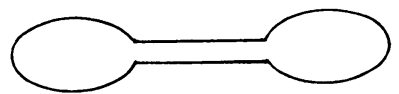

$\mathrm{x}=\frac{1}{2}$

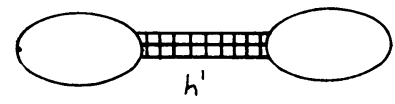

$x=\frac{1}{4}$

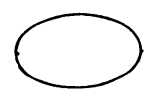

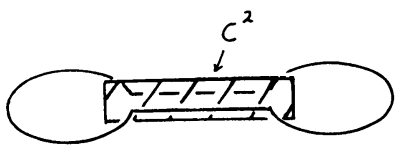
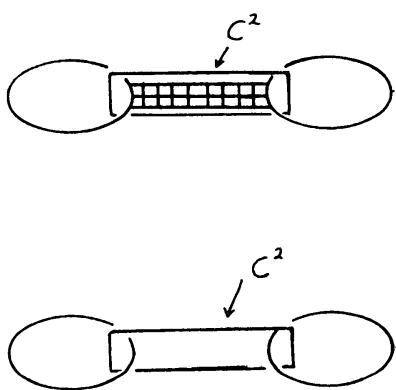

FIGURE 1 
In the case of a simple $2 q$-knot, $q \geqslant 2$, we can apply Corollary 10.1 of [K3] to obtain a presentation of the knot which contains only one 0 -handle of $S^{2 q}$, handles of index $q-1, q$, and $q+1$, and one $2 q$-handle. Moreover, the handles are added in order of increasing index, handles of the same index being added simultaneously.

Suppose that there are $m(q-1)$-handles of $S^{2 q}, n(q+1)$-handles, and hence $(m+n) q$-handles. Denote the $i$ th $r$-handle by $h_{i}^{r}$, and the induced $(r+1)$-handle of $K$ by $H_{i}^{r+1}$. Setting $X=h^{0} \cup \cup_{i=1}^{m} h_{i}^{q-1}$, it is shown in [K3] that the $h_{i}^{q-1}$ can all be added at the same level as $h^{0}$, and that $X$ is unknotted in $S^{2 q+1}$, in the sense of $\S 6$. Moving up to a level between the $h_{i}^{q-1}$ and the $h_{i}^{q}$, there is a copy of $\partial X$ unknotted in $S^{2 q+1}$ : let $P$ be its exterior, as in $\S 6$. Let $K^{q}$ denote the union of an embedded loop in $P$ representing $t \in \pi_{1}(P)=\pi_{1}(K)$, the $q$-spheres $X_{1}, \ldots, X_{m}$, together with embedded arcs joining the basepoint to each $X_{i}$ determined by the lift $\tilde{X}_{i}$. Then $K^{q}$ serves as a $q$-skeleton of $K$, for $X_{i}$ can be regarded as the core of $H_{i}^{q}$ with its attaching sphere deformation retracted into the 1-skeleton of $K$.

Let $P \times I$ be embedded productwise in $S^{2 q+1} \times I$, with $P \times 0$ identified with $P$, and $P \times 1$ contained in a level between $P$ and the $h_{i}^{q}$. Let $N\left(K^{q}\right)$ denote a regular neighbourhood of $K^{q}$ in $P$, and note that $\left(P, N\left(K^{q}\right)\right)$ is $q$-connected. The attaching sphere $S_{i}$ of $H_{i}^{q+1}$ can be taken as lying in $P \times 1$, and by engulfing we can arrange that $\cup_{i=1}^{m+n} S_{i} \subset N\left(K^{q}\right) \times 1$. Let $F: N\left(K^{q}\right) \times I \rightarrow N\left(K^{q}\right) \times I$ be a deformation retraction of $N\left(K^{q}\right)$ onto $K^{q}$, so that $F_{1}=$ identity, $F_{0}\left(N\left(K^{q}\right)\right)=K^{q}$. Then $F$ : $\cup_{i=1}^{m+n} S_{i} \times I \rightarrow N\left(K^{q}\right) \times I$ is an embedding except on $\cup_{i=1}^{m+n} S_{i} \times 0$. Let $C_{i}^{q+1}$ denote the union of the core of $H_{i}^{q+1}$ with $F\left(S_{i} \times I\right)$. Then $K^{q+1}=K^{q} \cup \cup_{i=1}^{m+n} C_{i}^{q+1}$ is a CW-complex which will serve as a $(q+1)$-skeleton of $K$.

If the handle $h_{i}^{q}$ is projected into the level containing $P$, then its image meets $P$ in a ball $k_{i}^{q}=\left(B^{q} \times B^{q}\right)_{i}$, with $\left(\partial B^{q} \times B^{q}\right)_{i} \subset \partial P$, and so choosing a lift $\left(\partial B^{q} \times 0\right)_{i}$ of $\left(\partial B^{q} \times 0\right)_{i}$ yields an element $\sum_{j=1}^{m} a_{i j}\left[\tilde{x}_{i}\right]$ of $H_{q}(\tilde{P}, \partial \tilde{P})$. The choice of lift induces a choice of $\tilde{C}_{i}^{q+1}$, and we have the following result.

LEMMA 7.1. The boundary map $H_{q+1}\left(\tilde{K}^{q+1}, \tilde{K}^{q}\right) \rightarrow H_{q}\left(\tilde{K}^{q}\right)$ is given by $\left[\tilde{C}_{i}^{q+1}\right] \mapsto$ $\sum_{j=1}^{m} a_{i j}\left[\tilde{X}_{j}\right]$.

Proof. Recall the way in which the attaching sphere of $H_{i}^{q+1}$ is related to the core of $h_{i}^{q}$; arguing as in the proof of [K3, Lemma 6.4], we have

$$
i_{*}\left[\partial \tilde{C}_{i}^{q+1}\right]=\sum_{j=1}^{m} a_{i j}(1-t)\left[\tilde{x}_{j}\right]=i_{*} \sum_{j=1}^{m} a_{i j}\left[\tilde{X}_{j}\right],
$$

and since $i_{*}$ is a monomorphism, this establishes the result.

LEMMA 7.2. Suppose that $\left[\tilde{C}_{i}^{q+1}\right], m<i \leqslant m+n$, are annihilated by the boundary map. Then the $h_{i}^{q}, m<i \leqslant m+n$, are unknotted.

Proof. Let $k_{i}^{q} \subset P$ be a handle as above, and $c_{i}^{q}$ its core. Since $c_{i}^{q}, m<i \leqslant m+n$, represents the zero element of $\pi_{q}(P, \partial P) \cong H_{q}(\tilde{P}, \partial \tilde{P})$ by the hypothesis, we can homotop $\cup_{i=m+1}^{m+n} c_{i}^{q}$ (rel $\partial$ ) by a homotopy $G$ until it is unknotted; that is, until each $c_{i}^{q}$ forms part of the boundary of a $(q+1)$-ball embedded in $P$, the rest of the boundary being in $\partial X \times * \subset \partial X \times S^{1}=\partial P$ for some point $* \in S^{1}$, and these balls 
being disjoint. We can assume that the homotopy $G$ has only transverse self-intersections, regarded as a map $G: \bigcup_{i=m+1}^{m+n} B_{i}^{q} \times I \rightarrow P \times I \subset K$. In an obvious way, $G$ induces a homotopy of $\bigcup_{i=m+1}^{m+n} k_{i}^{q}$ and hence a homotopy of $\cup_{i=m+1}^{m+n} S_{i}$ (recall that $S_{i}$ is the attaching sphere of $\left.H_{i}^{q+1}\right)$. The handles $k_{i}^{q}, m<i \leqslant m+n$, are unknotted now, except possibly for some twists, and so the homotopy of $\bigcup_{i=m+1}^{m+n} S_{i}$ may be finished off by a null-homotopy of each component, the only new singularities being self-intersections of each component corresponding to the number $d_{i}$ of twists in the $k_{i}^{q}$.

Let $f: \cup_{i=m+1}^{m+n} B_{i}^{q} \rightarrow P$ represent the initial embedding of $\bigcup_{i=m+1}^{m+n} c_{i}^{q}$, and $g$ the final embedding under the homotopy $G$. From [K1], there is an obstruction $d(f, g: G)$ to isotoping $f$ to $g$ (rel $\partial$ ), in the form of an $n \times n$ matrix over $\Lambda$, measured by the self-intersections of $G$. Let $S_{i}^{q+1}$ be the singular sphere formed by the union of the core of $H_{i}^{q+1}$ with the homotopy of $S_{i}$ and the final null-homotopy of $S_{i}$. Let $G_{i}=G \mid B_{i}^{q} \times I$. Then choosing the lifts determined by $\tilde{C}_{i}^{q+1}$, we have

$$
\begin{aligned}
S_{\tilde{K}}\left(\tilde{S}_{i}^{q+1}, \tilde{S}_{j}^{q+1}\right) & =(1-t)\left(1-t^{-1}\right) S_{\tilde{K}}\left(\tilde{G}_{i}, \tilde{G}_{j}\right)+2 \delta_{i j} d_{j} \\
& =(1-t)\left(1-t^{-1}\right) d(f, g: G)_{i, j}+2 \delta_{i j} d_{j} .
\end{aligned}
$$

But $\tilde{S}_{m+1}^{q+1}, \ldots, \tilde{S}_{m+n}^{q+1}$ is a (singular) set of generators for $H_{q+1}(\tilde{K})$; in fact, it is a set of generators for the free $\Lambda$-module $H_{q+1}\left(\tilde{K}^{q+1}\right)$. By the Blanchfield duality theorem [B] the intersection pairing on $H_{q+1}(\tilde{K})$ is zero, so $S_{\tilde{K}}\left(\tilde{S}_{i}^{q+1}, \tilde{S}_{j}^{q+1}\right)=0$ for each $i$ and $j$ from $m+1$ to $m+n$. Hence $d(f, g: G)=0$ and $d_{i}=0$ for $m+1 \leqslant i \leqslant m+$ $n$. Thus $f$ is isotopic to $g$ (rel d), by [K1], and so the $c_{i}^{q}$ are unknotted, and since $d_{i}=0$, the $k_{i}^{q}$ are unknotted.

8. Intersections and linking. Suppose that we have a simple presentation of a simple Z-torsion-free $2 q$-knot, $q \geqslant 6$. The associated handle decomposition of $K$ yields a chain complex $0 \rightarrow C_{q+2}(\tilde{K}) \rightarrow C_{q+1}(\tilde{K}) \rightarrow C_{q}(\tilde{K}) \rightarrow 0$ in which $C_{r}(\tilde{K})$ is a free $\Lambda$-module with basis given by the cores of the $H_{i}^{r}$. Corollary 4.5 shows that after stabilising this chain complex decomposes into two short exact sequences. This decomposition corresponds to a new choice of basis. The maps in the original chain complex are represented by matrices with respect to the bases mentioned above, and to get from these matrices to the matrices in the new sequence (with respect to the new bases), we must apply a sequence of matrix operations. These are the operations (i)-(iv) listed in $\$ 4$, together with:

(vi) Add a column of zeros to $A: A \mapsto(A 0)$.

(vii) Add a row of zeros to $A: A \mapsto\left(\begin{array}{c}A \\ 0\end{array}\right)$.

It is shown in [K3] that these matrix operations can be induced by handle moves of the knot presentation; that is, introducing trivial handle pairs and moving one handle over another of the same index. Thus we can arrange that the presentation has $m(q-1)$-handles $h_{i}^{q-1}(1 \leqslant i \leqslant m),(m+n) q$-handles $h_{i}^{q}(1 \leqslant i \leqslant m+n)$, and $n(q+1)$-handles $h_{m+i}^{q+1}(1 \leqslant i \leqslant n)$; moreover, the chain complex

$$
\begin{aligned}
& 0 \rightarrow C_{q+2}(\tilde{K}) \quad \rightarrow \quad C_{q+1}(\tilde{K}) \quad \rightarrow \quad C_{q}(\tilde{K}) \quad \rightarrow \quad 0 \\
& \| 2 \\
& 0 \rightarrow \Lambda^{n} \\
& \left(\begin{array}{ll}
B \\
0
\end{array}\right) \quad \| 2 \\
& \mathbb{R} \quad(0 A) \quad \| 2 \\
& \stackrel{(0 A)}{\rightarrow} \Lambda^{m} \quad \rightarrow \quad 0
\end{aligned}
$$


decomposes into the short exact sequences

$$
\begin{aligned}
& \Lambda^{n} \stackrel{B}{\rightarrow} \Lambda^{n} \rightarrow H_{q+1}(K), \\
& \Lambda^{m} \stackrel{A}{\rightarrow} \Lambda^{m} \rightarrow H_{q}(K) .
\end{aligned}
$$

In particular, by Lemma 7.2 , the $h_{m+i}^{q}(1 \leqslant i \leqslant n)$ are unknotted. Assume that $h_{i}^{q-1}(1 \leqslant i \leqslant m)$ and $h_{m+i}^{q}(1 \leqslant i \leqslant n)$ are added at the same level, and that $h_{i}^{q}$ $(1 \leqslant i \leqslant m)$ are added at a higher level. Let $S^{2 q+1}$ be a level between these two, and $Q \subset S^{2 q+1}$ the complement of the knot in that level. Then $Q$ has the properties described in $\$ 6$, and we shall use the notation developed there.

Consider now the dual presentation of the knot, in which the presentation is turned upside down and $r$-handles become $(2 q-r)$-handles. Let $k_{i}^{q-1}$ be dual to $h_{m+i}^{q+1}(1 \leqslant i \leqslant n), k_{i}^{q}$ dual to $h_{m+i}^{q}(1 \leqslant i \leqslant n), k_{n+i}^{q}$ dual to $h_{i}^{q}(1 \leqslant i \leqslant m)$, and $k_{n+i}^{q+1}$ dual to $h_{i}^{q-1}(1 \leqslant i \leqslant m)$. It is shown below that the corresponding chain complex yields short exact sequences

$$
\begin{aligned}
& \Lambda^{m} \stackrel{A^{*}}{\rightarrow} \Lambda^{m} \rightarrow H_{q+1}(\tilde{K}), \\
& \Lambda^{n} \stackrel{B^{*}}{\rightarrow} \Lambda^{n} \rightarrow H_{q}(\tilde{K})
\end{aligned}
$$

where $A^{*}$ denotes the conjugate transpose of $A$. Thus the $k_{b+i}^{q}(1 \leqslant i \leqslant m)$ are unknotted, and may be added at the same level as the $k_{i}^{q-1}$. In other words, we can add the $h_{m+i}^{q+1}$ at the same level as the $h_{i}^{q}(1 \leqslant i \leqslant m)$.

Recall that $H_{i}^{r+1}$ is the handle of $K$ induced by $h_{i}^{r}$, and $C_{i}^{r+1}$ the core of $H_{i}^{r+1}$. Let $K_{i}^{r+1}$ be the handle of $K$ induced by $k_{i}^{r}$, and $D_{i}^{r+1}$ the core of $K_{i}^{r+1}$. Consider a general presentation of $S^{n} \subset S^{n+1}$, with all the handles of the same index being added at the same level. Let $R$ be the level midway between the $h_{i}^{r}$ and the $h_{i}^{r+1}$. The following description is inherent in [K-L], and is illustrated in Figure 2 for the case $n=1, r=0$.

Writing $h_{i}^{r}=\left(B^{r} \times B^{n-r}\right)_{i}$, we can take as cocore of $H_{i}^{r+1}$ the ball

$$
I \times\left(0 \times(1-\varepsilon) B^{n-r}\right)_{i}=I \times E_{i}^{n-r}
$$

which meets $R$ in $E_{i}^{n-r}=1 \times\left(0 \times(1-\varepsilon) B^{n-r}\right)_{i}$. Note that the attaching sphere of $K_{i}^{n-r+1}$ is $D_{i}^{n-r+1}$, which is $\partial\left(\left(0 \times(1+\varepsilon) B^{n-r}\right)_{i} \times B^{1}\right)$ projected into $R$. Similarly, write $h_{j}^{r+1}=\left(B^{r+1} \times B^{n-r-1}\right)_{j}$ and take as cocore of $K_{j}^{n-r}$ the ball $I \times\left((1-\varepsilon) B^{r+1} \times 0\right)_{j}=I \times B_{j}^{r+1}$ which meets $R$ in

$$
B_{j}^{r+1}=0 \times\left((1-\varepsilon) B^{r+1} \times 0\right)_{j} .
$$

The attaching sphere of $H_{j}^{r+2}$ is $\partial C_{j}^{r+2}$, which is $\partial\left(\left((1+\varepsilon) B^{r+1} \times 0\right)_{j} \times B^{1}\right)$ projected into $R$.

Let $K^{(s)}$ denote the $s$-skeleton of $K$ in the handle decomposition of the $H_{i}^{l}$; that is, the union of all the $H_{i}^{l}$ for $l \leqslant s$. Similarly, let $K_{(s)}$ denote the $s$-skeleton of $K$ in the handle decomposition of the $K_{i}^{l}$. Let

$$
\begin{gathered}
\partial^{s}: H_{s+1}\left(\tilde{K}^{(s+1)}, \tilde{K}^{(s)}\right) \rightarrow H_{s}\left(\tilde{K}^{(s)}, \tilde{K}^{(s-1)}\right), \\
\partial_{s}: H_{s+1}\left(\tilde{K}_{(s+1)}, \tilde{K}_{(s)}\right) \rightarrow H_{s}\left(\tilde{K}_{(s)}, \tilde{K}_{(s-1)}\right)
\end{gathered}
$$

denote the boundary maps. 


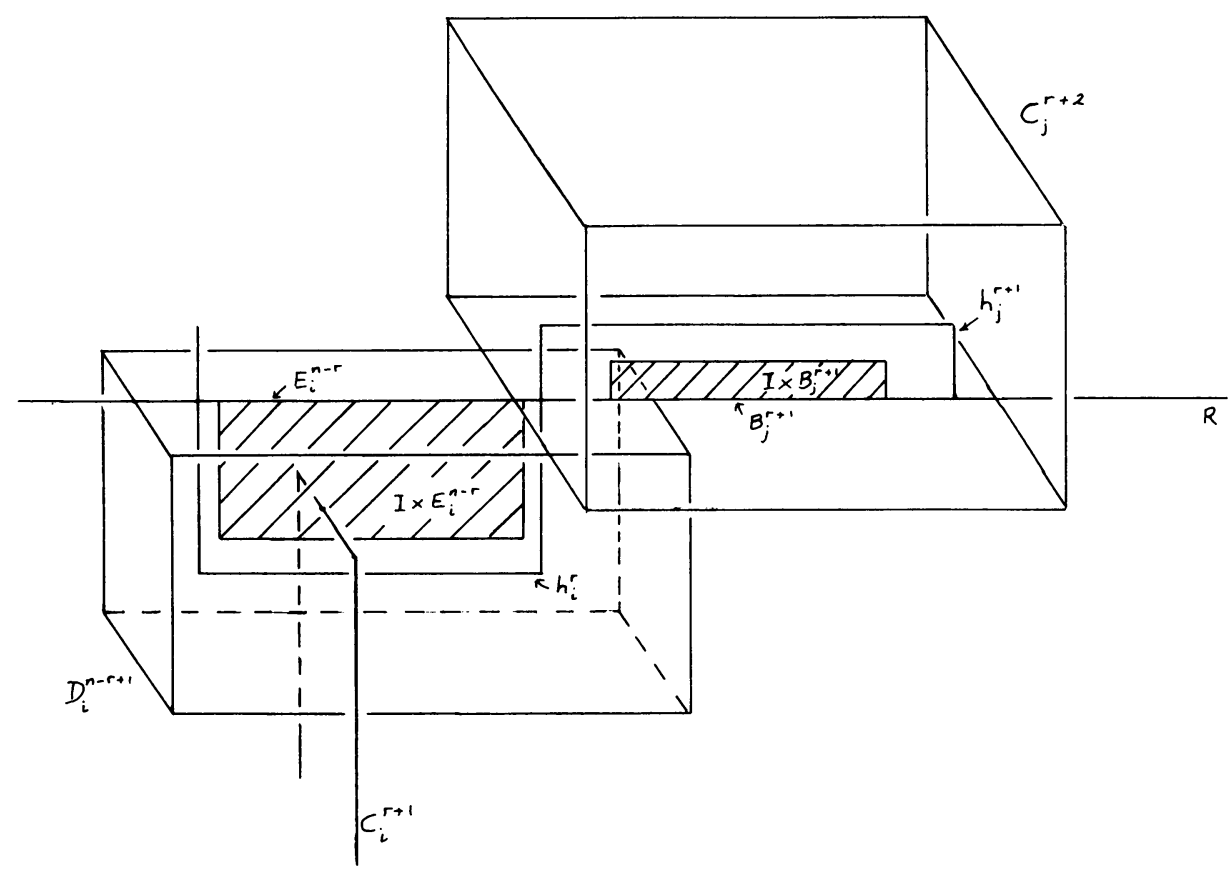

Figure 2

Choose lifts and orientations so that

$$
\begin{aligned}
& \partial^{r+1}\left[\tilde{C}_{i}^{r+2}\right]=\left(1-t^{\eta}\right)\left[\tilde{B}_{i}^{r+1}\right], \\
& \partial_{n-r}\left[\tilde{D}_{i}^{n-r+1}\right]=\left(1-t^{-\eta}\right)\left[\tilde{E}_{i}^{n-r}\right], \\
& S_{\tilde{K}}(\tilde{C}_{i}^{r+1}, \overbrace{I \times E_{j}^{n-r}}^{n-r}=\delta_{i j}, \\
& S_{\tilde{K}}\left(\tilde{D}_{i}^{n-r}, \overparen{I \times B_{j}^{r+1}}\right)=\delta_{i j}
\end{aligned}
$$

where $\eta= \pm 1$, depending on $r$ and $n$. Then

$$
\begin{gathered}
S_{\tilde{K}}\left(\tilde{C}_{i}^{r+1}, \tilde{D}_{j}^{n-r+1}\right)=\left(1-t^{\eta}\right) \delta_{i j}, \\
S_{\tilde{K}}\left(\tilde{D}_{i}^{n-r}, \tilde{C}_{j}^{r+2}\right)=\left(1-t^{-\eta}\right) \delta_{i j} .
\end{gathered}
$$

Define $\alpha_{i j}, \beta_{j i}(1 \leqslant i \leqslant k, 1 \leqslant j \leqslant l)$ by

$$
\begin{aligned}
\partial^{r+1}\left[\tilde{C}_{i}^{r+2}\right] & =\sum_{j=1}^{l} \alpha_{i j}\left[\tilde{C}_{j}^{r+1}\right], & 1 \leqslant i \leqslant k ; \\
\partial_{n-r}\left[\tilde{D}_{i}^{n-r+1}\right] & =\sum_{j=1}^{l} \beta_{i j}\left[\tilde{D}_{j}^{n-r}\right], & 1 \leqslant i \leqslant l .
\end{aligned}
$$

Then for $\varepsilon, \varepsilon^{\prime}= \pm 1$, depending on $n, r$, but not on $i, j$, we have

$$
\begin{aligned}
S_{\tilde{R}}\left(\partial^{r+1}\left[\tilde{C}_{i}^{r+2}\right],\left[\tilde{E}_{j}^{n-r}\right]\right) & =\varepsilon \alpha_{i j}, \\
S_{\tilde{R}}\left(\partial_{n-r}\left[\tilde{D}_{i}^{n-r+1}\right],\left[\tilde{B}_{j}^{r+1}\right]\right) & =\varepsilon^{\prime} \beta_{i j} .
\end{aligned}
$$


Thus

$$
\begin{aligned}
\varepsilon\left(1-t^{\eta}\right) \alpha_{i j} & =S_{\tilde{R}}\left(\partial^{r+1}\left[C_{i}^{r+2}\right],\left(1-t^{-\eta}\right)\left[\tilde{E}_{j}^{n-r}\right]\right) \\
& =S_{\tilde{R}}\left(\partial^{r+1}\left[C_{j}^{r+2}\right], \partial_{n-r}\left[\tilde{D}_{j}^{n-r+1}\right]\right) \\
& =(-1)^{(r+1)(n-r)} \frac{S_{\tilde{R}}\left(\partial_{n-r}\left[\tilde{D}_{j}^{n-r+1}\right], \partial^{r+1}\left[\tilde{C}_{i}^{r+2}\right]\right)}{S_{\tilde{R}}\left(\partial_{n-r}\left[\tilde{D}_{j}^{n-r+1}\right],\left(1-t^{\eta}\right)\left[\tilde{B}_{i}^{r+1}\right]\right)} \\
& =(-1)^{(r+1)(n-r)} \\
& =(-1)^{(r+1)(n-r)}\left(1-t^{\eta}\right) \varepsilon^{\prime} \bar{\beta}_{j i} ; \\
\alpha_{i j} & =\varepsilon^{\prime \prime} \bar{\beta}_{j i},
\end{aligned}
$$

where $\varepsilon^{\prime \prime}= \pm 1$ is independent of $i, j$.

Returning to the simple presentation of our $2 q$-knot, it follows easily that the dual presentation yields (up to sign) the short exact sequences as asserted above.

Computing the homology modules $H_{i}(\tilde{K}), i=q, q+1$, is a relatively simple matter, for the handle decomposition of $K$ in terms of the $H_{i}^{r}$ yields a presentation as above. In order to compute the Blanchfield duality pairing, the usual procedure would be to look at the dual handle decomposition of $(K, \partial K)$. Instead, we shall consider the handle decomposition $\left\{K_{i}^{r}\right\}$ obtained from the dual presentation of the knot.

Thus we have

$$
\left\langle\tilde{C}_{1}^{q}, \ldots, \tilde{C}_{m}^{q}:\right\rangle \stackrel{\phi}{\rightarrow}\left\langle\tilde{C}_{1}^{q}, \ldots, \tilde{C}_{m}^{q}: \sum_{j=1}^{m} a_{i j} \tilde{C}_{j}^{q}, 1 \leqslant i \leqslant m\right\rangle \cong H_{q}(\tilde{K})
$$

where $\partial^{q}\left[\tilde{C}_{i}^{q+1}\right]=\sum_{j=1}^{n} a_{i j}\left[\tilde{C}_{j}^{q}\right]$;

$$
\left\langle\tilde{C}_{m+1}^{q+1}, \ldots, \tilde{C}_{m+n}^{q+1}:\right\rangle \stackrel{\psi}{\rightarrow}\left\langle\tilde{C}_{m+1}^{q+1}, \ldots, \tilde{C}_{m+n}^{q+1}: \sum_{j=1}^{n} b_{i j} \tilde{C}_{m+j}^{q+1}, 1 \leqslant i \leqslant m\right\rangle \cong H_{q+1}(\tilde{K})
$$

where $\partial^{q+1}\left[\tilde{C}_{m+i}^{q+2}\right]=\sum_{j=1}^{n} b_{i j}\left[\tilde{C}_{m+j}^{q+1}\right]$;

$$
\left\langle\tilde{D}_{1}^{q}, \ldots, \tilde{D}_{n}^{q}:\right\rangle \stackrel{\phi^{\prime}}{\rightarrow}\left\langle\tilde{D}_{1}^{q}, \ldots, \tilde{D}_{n}^{q}: \sum_{j=1}^{n} b_{i j}^{*} \tilde{D}_{j}^{q}, 1 \leqslant i \leqslant n\right\rangle \cong H_{q}(\tilde{K})
$$

where $\partial_{q}\left[\tilde{D}_{i}^{q+1}\right]=\varepsilon \sum_{j=1}^{n} b_{i j}^{*}\left[\tilde{D}_{j}^{q}\right]$, and $b_{i j}^{*}=\bar{b}_{j i}$;

$$
\left\langle\tilde{D}_{n+1}^{q+1}, \ldots, \tilde{D}_{n+m}^{q+1}:\right\rangle \stackrel{\psi^{\prime}}{\rightarrow}\left\langle\tilde{D}_{n+1}^{q+1}, \ldots, \tilde{D}_{n+m}^{q+1}: \sum_{j=1}^{m} a_{i j}^{*} \tilde{D}_{n+j}^{q+1}, 1 \leqslant i \leqslant m\right\rangle \cong H_{q+1}(\tilde{K})
$$

where $\partial_{q+1}\left[\tilde{D}_{n+i}^{q+2}\right]=\varepsilon^{\prime} \sum_{j=1}^{m} a_{i j}^{*}\left[\tilde{D}_{n+j}^{q+1}\right]$. Note that $\varepsilon, \varepsilon^{\prime}= \pm 1$, depending on $q$ but not on $i, j$.

The intersection relations are

$$
\begin{aligned}
& S_{\tilde{K}}\left(\tilde{C}_{i}^{q+1}, \tilde{D}_{n+j}^{q+1}\right)=(1-t) \delta_{i j}, \quad 1 \leqslant i \leqslant m, 1 \leqslant j \leqslant m ; \\
& S_{\tilde{K}}\left(\tilde{C}_{m+i}^{q+1}, \tilde{D}_{j}^{q+1}\right)=\left(1-t^{-1}\right) \delta_{i j}, \quad 1 \leqslant i \leqslant n, 1 \leqslant j \leqslant n ;
\end{aligned}
$$

and zero otherwise;

$$
S_{\tilde{K}}\left(\tilde{D}_{i}^{q}, \tilde{C}_{m+j}^{q+2}\right)=\left(1-t^{-1}\right) \delta_{i j}, \quad 1 \leqslant i \leqslant n, 1 \leqslant j \leqslant n .
$$


LEMMA 8.1. $\left\langle\psi^{\prime} \tilde{D}_{n+i}^{q+1}, \phi \tilde{C}_{k}^{q}\right\rangle \equiv\left(1-t^{-1}\right)\left(A^{*-1}\right)_{i k} ;\left\langle\psi \tilde{C}_{m+i}^{q+1}, \phi \tilde{D}_{k}^{q}\right\rangle \equiv \varepsilon\left(1-t^{-1}\right) B_{i k}^{-1}$.

Proof. $\left\langle\psi^{\prime} \tilde{D}_{n+i}^{q+1}, \sum_{j=1}^{m} a_{k j} \phi \tilde{C}_{j}^{q}\right\rangle=S_{\tilde{K}}\left(\tilde{D}_{n+1}^{q+1}, \tilde{C}_{k}^{q+1}\right)=\left(1-t^{-1}\right) \delta_{i k}$, so

$$
\sum_{j=1}^{m}\left\langle\psi^{\prime} \tilde{D}_{n+i}^{q+1}, \phi \tilde{C}_{j}^{q}\right\rangle a_{j k}^{*}=\left(1-t^{-1}\right) \delta_{i k}
$$

and hence $\left\langle\psi^{\prime} \tilde{D}_{n+i}^{q+1}, \phi \tilde{C}_{k}^{q}\right\rangle \equiv\left(1-t^{-1}\right)\left(A^{*-1}\right)_{i k}$.

The second identity is proved similarly.

Recall the discussion in $§ 7$, where we used the $q$-spheres $X_{i}$ embedded in $P$ as part of a $q$-skeleton $K$ : by Lemma 7.1 , there is a presentation $\left\langle\tilde{X}_{1}, \ldots, \tilde{X}_{m}: \sum_{j=1}^{m} a_{i j} \tilde{X}_{j}\right.$, $1 \leqslant i \leqslant m\rangle$ of $H_{q}(\tilde{K})$, obtained in essentially the same way as $\left\langle\tilde{C}_{1}^{q}, \ldots, \tilde{C}_{m}^{q}\right.$ : $\left.\sum_{j=1}^{m} a_{i j} \tilde{C}_{j}^{q}, 1 \leqslant i \leqslant m\right\rangle$, for $X_{i}$ is just the core of $H_{i}^{q}$ with its attaching sphere deformation retracted to a point. In a similar manner we can use the spheres $Y_{i}, y_{i}^{*}$ embedded in $Q$ to obtain presentations

$$
\begin{aligned}
&\left\langle\tilde{Y}_{1}, \ldots, \tilde{Y}_{m}:\right\rangle \stackrel{\phi}{\rightarrow}\left\langle\tilde{Y}_{1}, \ldots, \tilde{Y}_{m}: \sum_{j=1}^{m} a_{i j} \tilde{Y}_{j}, 1 \leqslant i \leqslant m\right\rangle \cong H_{q}(\tilde{K}), \\
&\left\langle\tilde{y}_{m+1}^{*}, \ldots, \tilde{y}_{m+n}^{*}:\right\rangle \stackrel{\psi}{\rightarrow}\left\langle\tilde{y}_{m+1}^{*}, \ldots, \tilde{y}_{m+n}^{*}: \sum_{j=1}^{n} b_{i j} \tilde{y}_{m+j}^{*}, 1 \leqslant i \leqslant n\right\rangle \cong H_{q+1}(\tilde{K}) .
\end{aligned}
$$

The use of $\phi$ and $\psi$ is deliberate here, as for example $Y_{i}$ is essentially $C_{i}^{q}$ with its boundary deformed to a point.

The core of $h_{i}^{q}, 1 \leqslant i \leqslant m$, when projected into level $Q$, gives a $q$-ball $v_{i}$ properly embedded in $Q$. Arguing as in Lemma 7.1, $\left[\tilde{v}_{i}\right]=\sum_{j=1}^{m} a_{i j}\left[\tilde{y}_{j}\right]+\sum_{j=1}^{m} s_{i j}\left[\tilde{y}_{m+j}\right]$ for some matrix $S=\left(s_{i j}\right)$. Let $V_{i}$ be $\partial C_{i}^{q+1}$ projected into $Q, 1 \leqslant i \leqslant m$; then $i_{*}\left[\tilde{V}_{i}\right]=$ $(1-t)\left[\tilde{v}_{i}\right]=i_{*}\left(\sum_{j=1}^{m} a_{i j}\left[\tilde{Y}_{j}\right]+\sum_{j=1}^{n} s_{i j}\left[\tilde{Y}_{m+j}\right]\right)$, and since $i_{*}$ is a monomorphism, we have $\left[\tilde{V}_{i}\right]=\sum_{j=1}^{m} a_{i j}\left[\tilde{Y}_{j}\right]+\sum_{j=1}^{n} s_{i j}\left[\tilde{Y}_{m+j}\right]$ for $1 \leqslant i \leqslant m$.

The core of $h_{m+i}^{q+1}$, when projected into level $Q$, yields a $(q+1)$-ball $V_{m+i}^{*}$ properly (and permissibly) embedded in $Q$. An argument similar to that of Lemma 7.1 shows that for a suitable choice of lift,

$$
\left[\tilde{V}_{m+i}^{*}\right]=\sum_{j=1}^{m} c_{i j}\left[\tilde{Y}_{j}^{*}\right]+\sum_{j=1}^{n} b_{i j}\left[\tilde{Y}_{m+j}^{*}\right], \text { for some matrix } C=\left(c_{i j}\right),
$$

where orientations and lifts are chosen so that $\left[\partial \tilde{C}_{m+i}^{q+2}\right]=\left(1-t^{-1}\right)\left[\tilde{V}_{m+i}^{*}\right]$.

Because the dual handles $k_{i}^{q-1}, 1 \leqslant i \leqslant n$, and $k_{n+1}^{q}, 1 \leqslant i \leqslant m$, are unknotted, we can choose $q$-balls $v_{m+i}, 1 \leqslant i \leqslant n$, and $(q+1)$-balls $V_{i}^{*}, 1 \leqslant i \leqslant m$, properly (and permissibly) embedded in $Q$, and suitable lifts so that, defining $i_{*}\left(\tilde{V}_{i}\right)=$ $(1-t)\left(\tilde{v}_{i}\right), i_{*}\left(\tilde{v}_{i}^{*}\right)=\left(1-t^{-1}\right)\left(\tilde{V}_{i}^{*}\right)$, the homology classes $\left[\tilde{v}_{i}\right],\left[\tilde{V}_{i}^{*}\right],\left[\tilde{v}_{i}^{*}\right],\left[\tilde{V}_{i}\right]$ are related as in $\S 6$.

Looking at the dual presentation of the knot, we have presentations

$$
\begin{aligned}
&\left\langle\tilde{V}_{m+1}, \ldots, \tilde{V}_{m+n}:\right\rangle \stackrel{\phi^{\prime}}{\rightarrow}\left\langle\tilde{V}_{m+1}, \ldots, \tilde{V}_{m+n}: \sum_{j=1}^{n} b_{i j}^{*} \tilde{V}_{m+j}, 1 \leqslant i \leqslant n\right\rangle \cong H_{q}(\tilde{K}), \\
&\left\langle\tilde{v}_{1}^{*}, \ldots, \tilde{v}_{m}^{*}:\right\rangle \stackrel{\psi^{\prime}}{\rightarrow}\left\langle\tilde{v}_{1}^{*}, \ldots, \tilde{v}_{m}^{*}: \sum_{j=1}^{m} a_{i j}^{*} \tilde{v}_{j}^{*}, 1 \leqslant i \leqslant m\right\rangle \cong H_{q+1}(\tilde{K})
\end{aligned}
$$


where $\tilde{V}_{m+i}$ corresponds to $\tilde{D}_{i}^{q}$ with its boundary shrunk to a point, and $\tilde{v}_{i}^{*}$ to $\tilde{D}_{n+i}^{q+q}$. Note that since $\tilde{v}_{m+i}^{*}$ is null-homologous in $\tilde{K}$, we could add these as generators, with $\psi^{\prime} \tilde{v}_{m+i}^{*}=0$.

Thus Lemma 8.1 becomes

LEMMA 8.1'.

$$
\begin{gathered}
\left\langle\psi^{\prime} \tilde{v}_{i}^{*}, \phi \tilde{Y}_{k}\right\rangle \equiv\left(1-t^{-1}\right)\left(A^{-1}\right)_{i k}, \\
\left\langle\psi \tilde{y}_{m+i}^{*}, \phi^{\prime} \tilde{V}_{m+k}\right\rangle \equiv \varepsilon\left(1-t^{-1}\right) B_{i k}^{-1}
\end{gathered}
$$

LEMMA 8.2.

$$
\left\langle\psi \tilde{y}_{m+i}^{*}, \phi \tilde{Y}_{k}\right\rangle \equiv\left(1-t^{-1}\right)\left(S^{*} A^{*-1}\right)_{i k} .
$$

Proof. By Lemma 6.16 we have

$$
\begin{aligned}
\left\langle\psi \tilde{y}_{m+i}^{*}, \phi \tilde{Y}_{k}\right\rangle & \equiv\left\langle\psi^{\prime}\left(\sum_{j=1}^{m} s_{i j}^{*} \tilde{v}_{j}^{*}+\sum_{j=1}^{n} q_{i j}^{*} \tilde{v}_{m+j}^{*}\right), \phi \tilde{Y}_{k}\right\rangle \\
& \equiv \sum_{j=1}^{m} s_{i j}^{*}\left\langle\psi^{\prime} \tilde{v}_{j}^{*}, \phi \tilde{Y}_{k}\right\rangle \\
& \equiv\left(1-t^{-1}\right)\left(S^{*} A^{*-1}\right)_{i k} .
\end{aligned}
$$

LEMMA 8.3. Let $y_{i}$ be altered to $y_{i}$, as in Lemma 6.7, and let $\underline{A}, \underline{S}$ be the matrices corresponding to $A, S$ respectively with the new basis. Then $\underline{A}=A$ and $\underline{S}=S-A V$.

PROOF. $\left[\tilde{y}_{i}\right]=\left[\tilde{y}_{i}\right]+\sum_{j=1}^{n} v_{i j}\left[\tilde{y}_{m+j}\right], 1 \leqslant i \leqslant m$, by Lemma 6.7.

$$
\begin{array}{ll}
{\left[\underline{\tilde{v}}_{i}\right]=\sum_{j=1}^{m} \underline{a}_{i j}\left[\tilde{y}_{j}\right]+\sum_{j=1}^{n} \underline{s}_{i j}\left[\tilde{y}_{m+j}\right],} & 1 \leqslant i \leqslant m, \\
{\left[\tilde{v}_{i}\right]=\sum_{j=1}^{m} a_{i j}\left[\tilde{y}_{i}\right]+\sum_{j=1}^{n} s_{i j}\left[\tilde{y}_{m+j}\right],} & 1 \leqslant i \leqslant m .
\end{array}
$$

By the hypothesis, $\left[\tilde{y}_{m+i}\right]=\left[\tilde{y}_{m+i}\right]$ for $1 \leqslant i \leqslant n$, and since $\left[\underline{\tilde{v}}_{i}\right]=\left[\tilde{v}_{i}\right]$ for each $i$, we obtain

$$
\begin{aligned}
0= & \sum_{j=1}^{m}\left(\underline{a}_{i j}-a_{i j}\right)\left[\tilde{y}_{j}\right]+\sum_{j=1}^{m} \underline{a}_{i j} \sum_{k=1}^{n} v_{j k}\left[\tilde{y}_{m+k}\right] \\
& +\sum_{j=1}^{n}\left(\underline{s}_{i j}-s_{i j}\right)\left[\tilde{y}_{m+j}\right], \quad 1 \leqslant i \leqslant m .
\end{aligned}
$$

Equating coeffients gives $\underline{A}=A$, and $A V+\underline{S}-S=0$.

9. Proof of Theorem 2.2. Assume that we are given two $2 q$-knots $k_{1}, k_{2}$, each of which is a simple Z-torsion-free knot. Assume also that $q \geqslant 6$ and that the two knots have isometric $F$-forms. Take a simple presentation of each knot $k_{i}$, giving rise as in $\S 8$ to the matrices $A_{i}, B_{i}$, which present $H_{q}\left(\tilde{K}_{i}\right), H_{q+1}\left(\tilde{K}_{i}\right)$. By Proposition 3.1 , the augmented $F$-forms of $k_{1}$ and $k_{2}$ are isometric. By Lemma 4.7, after a sequence of matrix moves we can assume $A_{1}=A_{2}=A, B_{1}=B_{2}=B$; moreover, if $\phi_{i}$ : $\Lambda^{m} \rightarrow H_{q}\left(\tilde{K}_{i}\right), \psi_{i}: \Lambda^{n} \rightarrow H_{q+1}\left(\tilde{K}_{i}\right)$ are the quotient maps, and $a: H_{q}\left(\tilde{K}_{1}\right) \rightarrow H_{q}\left(\tilde{K}_{2}\right)$, $c: H_{q+1}\left(\tilde{K}_{1}\right) \rightarrow H_{q+1}\left(\tilde{K}_{2}\right)$ are the isomorphisms of the isometry, then we may 
assume that $a \phi_{i}=\phi_{2}, c \psi_{i}=\psi_{2}$. Since the matrix moves involved can be induced by handle moves in the knot presentation (compare [K3]), we obtain presentations of $k_{1}, k_{2}$, in which the 0 -handles coincide, the $(q-1)$-handles $h_{i}^{q-1}(1 \leqslant i \leqslant m)$ coincide, and the $h_{m+i}^{q}(1 \leqslant i \leqslant n)$ coincide. Next we show that the $h_{i}^{q}(1 \leqslant i \leqslant m)$ may be isotoped until they coincide.

Since the pairings $\langle\rangle:, H_{q+1}(\tilde{K}) \times H_{q}(\tilde{K}) \rightarrow \Lambda_{s} / \Lambda$ are isometric, we know from Lemma 8.2 that

$$
\left(1-t^{-1}\right) S_{1}^{*} A^{*-1} \equiv\left(1-t^{-1}\right) S_{2}^{*} A^{*-1} \quad(\bmod \Lambda),
$$

and so $\left(1-t^{-1}\right) S_{1}^{*} A^{*-1}=\left(1-t^{-1}\right) S_{2}^{*} A^{*-1}+M$ where $M$ is an $n \times m$ matrix over $\Lambda$. Thus

$$
\begin{aligned}
\left(1-t^{-1}\right) S_{1}^{*} & =\left(1-t^{-1}\right) S_{2}^{*}+M A^{*} \\
(1-t) S_{1} & =(1-t) S_{2}+A M^{*}
\end{aligned}
$$

Now $\operatorname{det} A$ is the Alexander polynomial of $k_{1}$ in dimension $q$, so $(1-t)$ is prime to det $A$. But we have an equation $A M^{*}=(1-t)\left(S_{1}-S_{2}\right)$, from which

$$
M^{*}=(1-t) \cdot \frac{\operatorname{adj} A}{\operatorname{det} A} \cdot\left(S_{1}-S_{2}\right)
$$

Because $(1-t)$ does not divide $\operatorname{det} A, \operatorname{det} A$ must divide adj $A \cdot\left(S_{1}-S_{2}\right)$, and so $(1-t)$ divides $M^{*}$. Write $M^{*}=(1-t) N$, and we have $S_{1}=S_{2}+A N$. Taking $V=-N$ in Lemma 8.3, we can by a change of basis arrange that $S_{1}=S_{2}$. Note that such a change of basis does not affect $A, B, \phi, \psi$. Corollary 6.9 shows that there is an ambient isotopy $F$ such that in the presentation of $F\left(k_{2}\right)$, the $(q-1)$-handles of $F\left(k_{2}\right)$ coincide with those of $k_{1}$, the last $n q$-handles of $F\left(k_{2}\right)$ coincide with those of $k_{1}$, and $S_{1}=S_{2}=S$.

Recalling the definition of $A$ and $S$ in terms of the handles $h_{i}^{q}(1 \leqslant i \leqslant m)$, it follows that the cores of the handles of $k_{2}$ represent the same elements of $\pi_{q}(Q, \partial Q)$ as the corresponding cores for $k_{1}$. By the proof of Lemma 7.3 in [K3], quoting Irwin's Theorem in place of general position, we may by an ambient isotopy arrange that corresponding cores coincide on $\partial Q$ and are homotopic rel $\partial$.

But the cores of the $h_{i}^{q}(1 \leqslant i \leqslant m)$ form part of a basis for $H_{q}(\tilde{Q}, \partial \tilde{Q})$ (consider the dual presentation of the knot), and so the results of $\$ 5$ and [K1] show that $k_{2}$ can be ambient isotoped until all its $(q-1)$ and $q$-handles coincide with those of $k_{1}$.

Let the $(q+1)$-handles of $k$ be $h_{m+1}^{q+1}, \ldots, h_{m+n}^{q+1}$; when projected into level $Q$, the core of $h_{m+i}^{q+1}$ yields a ball $V_{m+i}^{*}$ permissibly embedded in $Q$, with

$$
\left[\tilde{V}_{m+i}^{*}\right]=\sum_{j=1}^{m} c_{i j}\left[\tilde{Y}_{j}^{*}\right]+\sum_{j=1}^{n} b_{i j}\left[\tilde{Y}_{m+j}^{*}\right] .
$$

If the knot $k_{i}$ gives rise to the matric $C_{i}$, then by the results of $\S 6$, and in particular Lemma 6.14, each $C_{i}$ satisfies the equation $C A^{*}+B S^{*}=0$. Since $A^{*}$ is nonsingular, $C_{1}=C_{2}=C$.

We adopt the notation of $\S 8$ with regard to $\tilde{V}_{i}, \tilde{V}_{i}^{*}$, etc., without further comment. Recall that $(\tilde{Y})$ denotes the homotopy class of the ball $\tilde{Y}$ properly embedded in $\tilde{Q}$. 
For $1 \leqslant i \leqslant n$, we have

$$
\left(\tilde{V}_{m+i}^{*}\right)=\sum_{j=1}^{m} c_{i j}\left(\tilde{Y}_{j}^{*}\right)+\sum_{j=1}^{n} b_{i j}\left(\tilde{Y}_{m+j}^{*}\right)+\sum_{j=1}^{m} d_{i j}\left(\tilde{y}_{j}\right) \circ \xi+\sum_{j=1}^{n} e_{i j}\left(\tilde{y}_{m+j}\right) \circ \xi
$$

where $D, E$ are matrices over $\Lambda$, defined $\bmod 2$.

Then

$$
\begin{aligned}
\left(\partial \tilde{C}_{m+i}^{q+2}\right) & =\left(1-t^{-1}\right)\left(\tilde{V}_{m+i}^{*}\right) \\
& =\sum_{j=1}^{m} c_{i j}\left(\tilde{y}_{j}^{*}\right)+\sum_{j=1}^{n} b_{i j}\left(\tilde{y}_{m+j}^{*}\right)-t^{-1} \sum_{j=1}^{m} d_{i j}\left(\tilde{Y}_{j}\right) \circ \zeta-t^{-1} \sum_{j=1}^{n} e_{i j}\left(\tilde{Y}_{m+j}\right) \circ \zeta .
\end{aligned}
$$

Arguing as in $\S 8$, there is a presentation of $\pi_{q+1}(\tilde{K})$ as a $\Lambda$-module:

$$
\begin{aligned}
\left\langle\tilde{Y}_{1}, \ldots, \tilde{Y}_{m}, \tilde{y}_{m+1}^{*}, \ldots, \tilde{y}_{m+n}^{*}: 2 \tilde{Y}_{1}, \ldots, 2 \tilde{Y}_{m},\right. & \sum_{j=1}^{m} a_{i j} \tilde{Y}_{j}(1 \leqslant i \leqslant m), \\
& \left.-t^{-1} \sum_{y=1}^{m} d_{i j} \tilde{Y}_{j}+\sum_{j=1}^{n} b_{i j} \tilde{y}_{m+j}^{*}(1 \leqslant i \leqslant n)\right\rangle .
\end{aligned}
$$

Let $\kappa_{i}:\left\langle\tilde{Y}_{1}, \ldots, \tilde{Y}_{m}, \tilde{y}_{m+1}^{*}, \ldots, \tilde{y}_{m+n}^{*}:\right\rangle \rightarrow \pi_{q+1}\left(\tilde{K}_{i}\right)$ be the quotient maps, for $i=1,2$, and let $b: \pi_{q+1}\left(\tilde{K}_{1}\right) \stackrel{\sim}{\rightarrow} \pi_{q+1}\left(\tilde{K}_{2}\right)$ be the isometry. Since $a \phi_{1}=\phi_{2}$, we know that $b \kappa_{1}\left(\tilde{Y}_{i}\right)=\kappa_{2}\left(\tilde{Y}_{i}\right)$ for $1 \leqslant i \leqslant m$. Similarly, since $c \psi_{1}=\psi_{2}$, we know that $b \kappa_{1}\left(\tilde{y}_{m+i}^{*}\right)-\kappa_{2}\left(\tilde{y}_{m+i}^{*}\right) \in \omega\left(H_{q}\left(\tilde{K}_{2}\right)\right)=\kappa_{2}\left(\left\langle\tilde{Y}_{1}, \ldots, \tilde{Y}_{m}\right\rangle\right)$. Say $b \kappa_{1}\left(\tilde{y}_{m+i}^{*}\right)=$ $\kappa_{2}\left(\tilde{y}_{m+i}^{*}+\sum_{j=1}^{m} \sigma_{i j} \tilde{Y}_{j}\right)$. Then

$$
\begin{aligned}
i_{*}\left(\left(\tilde{y}_{m+i}^{*}\right)+\sum_{j=1}^{m} \sigma_{i j}\left(\tilde{Y}_{j}\right) \circ \zeta\right)=\left(1-t^{-1}\right)\left(\tilde{Y}_{m+i}^{*}\right)+(1-t) \sum_{j=1}^{m} \sigma_{i j}\left(\tilde{y}_{j}\right) \circ \xi \\
=\left(1-t^{-1}\right)\left\{\left(\tilde{Y}_{m+i}^{*}\right)-t^{-1} \sum_{j=1}^{m} \sigma_{i j}\left(\tilde{y}_{j}\right) \circ \xi\right\}=\left(1-t^{-1}\right)\left(\tilde{Y}_{m+i}^{*}\right) .
\end{aligned}
$$

Consider the $q$-handles $h_{m+i}^{q}(1 \leqslant i \leqslant n)$ projected into level $P$ : then the sets $\left\{\left(\tilde{Y}_{m+i}^{*}\right)\right\}_{1 \leqslant i \leqslant n}$ and $\left\{\left(\tilde{Y}_{m+i}^{*}\right)\right\}_{1 \leqslant i \leqslant n}$ can each be represented by a set of $(q+1)$-balls which "cancel" the $h_{m+i}^{q}$, and there is a permissible ambient isotopy of $P$ which takes $h_{m+i}^{q}$ back to its starting position, and $Y_{m+i}^{*}$ onto $Y_{m+i}^{*}$. In other words, we can by temporarily adding the $h_{i}^{q-1}$ before the $h_{i}^{q}$, isotop the $q$-handles of $k_{2}$ so as to make $b \kappa_{1}\left(\tilde{y}_{m+i}^{*}\right)=\kappa_{2}\left(\tilde{y}_{m+i}^{*}\right)$, that is $b \kappa_{1}=\kappa_{2}$.

Let $k_{1}$ give rise to $D, k_{2}$ to $D^{+}:$then for $1 \leqslant i \leqslant n$ :

$$
\begin{aligned}
b \kappa_{1}\left(-t^{-1} \sum_{j=1}^{m} d_{i j} \tilde{Y}_{j}+\sum_{j=1}^{m} b_{i j} \tilde{y}_{m+j}^{*}\right) & =0, \\
\kappa_{2}\left(-t^{-1} \sum_{j=1}^{m} d_{i j}^{+} \tilde{Y}_{j}+\sum_{j=1}^{m} b_{i j} \tilde{y}_{m+j}^{*}\right) & =0
\end{aligned}
$$

and so

$$
\kappa_{2}\left(\sum_{j=1}^{m}\left(d_{i j}-d_{i j}^{+}\right) \tilde{Y}_{j}\right)=0
$$


Since $B$ is nonsingular, and $D$ is only defined $\bmod 2$, there exist $\mu_{i j} \in \Lambda$ such that

and so $D-D^{+}=M A$.

$$
\sum_{j=1}^{m}\left(d_{i j}-d_{i j}^{+}\right) \tilde{Y}_{j}=\sum_{j=1}^{m} \sum_{k=1}^{m} \mu_{i j} a_{j k} \tilde{Y}_{k}, \quad 1 \leqslant i \leqslant n ;
$$

LemMA 9.3. The $(q+1)$-handles of $k_{2}$ can be isotoped to make $D^{+}=D$.

Proof. Move the $(q+1)$-handles $h_{m+i}^{q+1}$ to a higher level than the $h_{i}^{q}$, and let $R$ be a level between these two. Let $B_{i}^{q+1}$ be $\left((1+\varepsilon) B^{q} \times 0 \times B^{1}\right)_{i}$ projected into level $R$, where $\left(B^{q} \times B^{q}\right)_{i}$ is $h_{i}^{q} \cap Q$. Let $\zeta_{i}: S^{q+1} \rightarrow \partial B_{i}^{q+1}$ represent the nontrivial element of $\pi_{q+1}\left(S^{q}\right)$. Let $\omega_{i}: B^{q+1} \rightarrow R$ correspond to $V_{m+i}^{*}, 1 \leqslant i \leqslant n$. For $1 \leqslant i \leqslant n$, $1 \leqslant j \leqslant m$, we can choose an arc joining $\operatorname{Im} \omega_{i}$ and $\operatorname{Im} \zeta_{j}$ in $R$, and by taking the interior connected sum obtain a new embedding $\omega_{i j}: B^{q+1} \rightarrow R$ which projects to a map $\tau_{i j}: B^{q+1} \rightarrow Q$. It is clear that $\omega_{i}$ and $\omega_{i j}$ are isotopic rel $\partial$, since $\zeta_{i}$ is null-homotopic as a map $S^{q+1} \rightarrow R$, but $\tau_{i j}$ represents $\left(\tilde{V}_{m+i}^{*}\right)+t^{\alpha}\left(\tilde{V}_{j}\right)=\left(V_{m+i}^{*}\right)+$ $t^{\alpha}(1-t)\left(\tilde{v}_{j}\right) \circ \xi$ where $\alpha$ depends on the choice of the arc mentioned above. Thus, by the construction, we can isotop $h_{m+i}^{q+1}$ rel $\partial$ in level $R$ so that its core when projected down to level $Q$, instead of representing $\left(\tilde{V}_{m+i}^{*}\right)$, represents

$$
\begin{aligned}
\left(\tilde{V}_{m+i}^{*}\right)+t^{\alpha}(1-t) & \left(\tilde{v}_{j}\right) \circ \xi \\
= & \left(\tilde{V}_{m+i}^{*}\right)+t^{\alpha}(1-t)\left\{\sum_{k=1}^{m} a_{j k}\left(\tilde{y}_{k}\right) \circ \xi+\sum_{k=1}^{m} s_{j k}\left(\tilde{y}_{m+k}\right) \circ \xi\right\} .
\end{aligned}
$$

Thus by isotopies of this kind we may move the $h_{m+i}^{q+1}$ of $k_{2}$ until $M$ is a matrix over Z.

A similar argument, allowing the attaching tubes of the $h_{m+i}^{q+1}$ to move within $\left[\#_{1}^{n}\left(S^{q} \times S^{q-1}\right)\right] \times * \subset\left[\#_{1}^{n}\left(S^{q} \times S^{q-1}\right)\right] \times S^{1}=\partial R$, completes the proof.

Let $Q^{\prime}$ be a level just above $Q$, but below the level of the $h_{i}^{q}(1 \leqslant i \leqslant m)$, and let $y_{i}^{\prime *}(1 \leqslant i \leqslant m+n)$ be copies of $y_{i}^{*}$ embedded in $Q^{\prime}$. In order to compute the homotopy pairing $\{$,$\} we shall need to look at the intersection of \tilde{C}_{i}^{q+2}$ and $\tilde{y}_{j}^{\prime *}$ in $\tilde{K}$, and since $\tilde{C}_{i}^{q+2}$ meets $\tilde{Q}^{\prime}$ in $\left(1-t^{-1}\right)\left(\tilde{V}_{i}^{\prime *}\right)$, this can be done by looking at the intersection of $\left(1-t^{-1}\right)\left(\tilde{V}_{i}^{\prime *}\right)$ and $\left(\tilde{y}_{j}^{*}\right)$ in $\tilde{Q}^{\prime}$, or equivalently the intersection of $\left(1-t^{-1}\right)\left(\tilde{V}_{i}^{*}\right)$ and $\left(\tilde{y}_{j}^{*}\right)$ in $\tilde{Q}$.

Recall from $\S 1$ the way in which the pairings $\{$,$\} and T$ are defined.

$$
\begin{aligned}
& -t^{-1} \sum_{j=1}^{m} d_{i j}\left\{\kappa \tilde{Y}_{j}, \kappa \tilde{y}_{m+k}^{*}\right\}+\sum_{j=1}^{n} b_{i j}\left\{\kappa \tilde{y}_{m+j}^{*}, \kappa \tilde{y}_{m+k}^{*}\right\} \\
& =\left\{\partial \tilde{C}_{m+i}^{q+2}, \kappa \tilde{y}_{m+k}^{*}\right\}=T_{\tilde{K}}\left(\tilde{C}_{m+i}^{q+2}, \tilde{y}_{m+k}^{\prime *}\right) \\
& =T_{\tilde{Q}^{\prime}}\left(\left(1-t^{-1}\right)\left(\tilde{V}_{m+i}^{\prime *}\right),\left(\tilde{y}_{m+k}^{\prime *}\right)\right) \\
& =T_{\tilde{Q}}\left(\left(1-t^{-1}\right) \sum_{j=1}^{m} c_{i j}\left(\tilde{Y}_{j}^{*}\right)+\left(1-t^{-1}\right) \sum_{j=1}^{n} b_{i j}\left(\tilde{Y}_{m+j}^{*}\right)\right. \\
& \left.\quad+\left(1-t^{-1}\right) \sum_{j=1}^{m} d_{i j}\left(\tilde{y}_{j}\right) \circ \xi+\left(1-t^{-1}\right) \sum_{j=1}^{n} e_{i j}\left(\tilde{y}_{m+j}\right) \circ \xi,\left(\tilde{y}_{m+k}\right)\right) \\
& =\left(1-t^{-1}\right) e_{i k},
\end{aligned}
$$

using the equations (6.1). 
Set $u_{j k}=\left\{\kappa \tilde{y}_{m+j}^{*}, \kappa \tilde{y}_{m+k}^{*}\right\}$ and note from Lemma 8.2 that

$$
\left\{\kappa \tilde{Y}_{j}, \kappa \tilde{y}_{m+k}^{*}\right\} \equiv \theta\left\langle h_{q+1} \kappa \tilde{Y}_{j}, h_{q+1} \kappa \tilde{y}_{m+k}^{*}\right\rangle \equiv \theta(1-t)\left(A^{-1} S\right)_{j k} .
$$

From the calculation above we obtain

$$
-t^{-1}(1-t) D A^{-1} S+B U=\left(1-t^{-1}\right) E,
$$

the matrices having entries in $\Gamma_{0}$.

Let $E_{i}, U_{i}$ be the matrices $E, U$ arising from the presentation of $k_{i}$. Since the pairings are isometric, we have $U_{1} \equiv U_{2}(\bmod \Gamma)$. It follows that

$$
\left(1-t^{-1}\right) B^{-1}\left(E_{1}-E_{2}\right)=U_{1}-U_{2}=F,
$$

where $F$ has entries in $\Gamma$. Since $B^{-1}=\operatorname{adj} B / \operatorname{det} B$, and $(1-t)$ is prime to det $B$, we have $B^{-1}\left(E_{1}-E_{2}\right)=G$ where $G$ is a matrix over $\Gamma$. Thus $E_{1}-E_{2}=B G$. Taking $G=-\alpha$ in Lemma 6.12, which we may do becuase the $V_{i}^{*}$ are embedded for each knot, we can isotop the $q$-handles of $k_{2}$ to make $E_{1}=E_{2}$. Then $k_{1}$ and $k_{2}$ coincide up to and including their $q$-handles, and the cores of their $(q+1)$-handles are homotopic rel $\partial$. Since they form part of a basis for $H_{q+1}(\tilde{Q}, \partial \tilde{Q})$, they are isotopic rel $\partial$ by Proposition 5.1 of [K1]. By Lemma 5.3, the $(q+1)$-handles of $k_{2}$ are permissibly isotopic to those of $k_{1}$, and so it only remains to isotop the $2 q$-handle of $k_{2}$ onto that of $k_{1}$. If the $2 q$-handle of each knot is added in level $S^{2 q+1} \times 1 \subset$ $S^{2 q+1} \times I$, then we can push the interior of the $2 q$-handle into the interior of the $B^{2 q+2}$ which must be attached to $S^{2 q+1} \times 1$. Thus we have two unknotted ball pairs $\left(B^{2 q+2}, B^{2 q}\right)$ which agree along the boundary, and these are ambient isotopic rel $\partial$ by the Alexander trick.

10. Proof of Theorem 2.3. Assume the data of the theorem: by Propositions 3.1 and 3.2 , there is an augmented $F$-form associated to the given $F$-form, and this is unique up to isometry. We shall construct a knot which realises the augmented $F$-form.

By Lemma II.12 of [Ke], the modules $H_{q}, H_{q+1}$ are presented by nonsingular matrices $A, B$ respectively, where $A$ is an $m \times m$ matrix over $\Lambda$ and $B$ is an $n \times n$ matrix over $\Lambda$. In this context, nonsingular means that the matrix has nonzero determinant.

Let $\left\langle\tilde{Y}_{1}, \ldots, \tilde{Y}_{m}: \sum_{j=1}^{m} a_{i j} \tilde{Y}_{j}, \quad 1 \leqslant i \leqslant m\right\rangle$ be the presentation of $H_{q}$, and $\left\langle\tilde{y}_{m+1}^{*}, \ldots, \tilde{y}_{m+n}^{*}: \sum_{j=1}^{n} b_{i j} \tilde{y}_{m+j}^{*}, 1 \leqslant i \leqslant n\right\rangle$ the presentation of $H_{q+1}$, with

$$
\phi:\left\langle\tilde{Y}_{1}, \ldots, \tilde{Y}_{m}:\right\rangle \rightarrow H_{q} \text { and } \psi:\left\langle\tilde{y}_{m+1}^{*}, \ldots, \tilde{y}_{m+n}^{*}:\right\rangle \rightarrow H_{q+1}
$$

the quotient maps.

Recall that $(1-t): H_{q} \rightarrow H_{q}$ is an isomorphism, and set $\phi \tilde{Y}_{k}=(1-t) \phi \tilde{Z}_{k}$. For each $i, k$, choose lifts of $\left\langle\psi \tilde{y}_{m+i}^{*}, \phi \tilde{Z}_{k}\right\rangle \in \Lambda_{S} / \Lambda$ to $\Lambda_{S}$. Define the $m \times n$ matrix $S$ by the equation

$$
s_{i j}^{*}=\sum_{k=1}^{m} \bar{a}_{j k}\left\langle\psi \tilde{y}_{m+i}^{*}, \phi \tilde{Z}_{k}\right\rangle \in \Lambda_{s}
$$

Note that $(\bmod \Lambda)$

$$
\left(1-t^{-1}\right) s_{i j}^{*} \equiv\left\langle\psi \tilde{y}_{m+i}^{*}, \sum_{k=1}^{m} \phi\left(a_{j k} \tilde{Y}_{k}\right)\right\rangle \equiv 0,
$$


and so $S$ has entries in $\Lambda$. Comparison with Lemma 8.2 shows that

$$
\left\langle\psi y_{m+i}^{*}, \phi Y_{k}\right\rangle \equiv\left(1-t^{-1}\right)\left(S^{*} A^{*-1}\right)_{i k} .
$$

Next we define the matrix $C$ by the equation

$$
C A^{*}+B S^{*}=0
$$

since det $A \neq 0$, this determines $C$ as a matrix over $\Lambda_{0}$. In fact, $C$ is a matrix over $\Lambda$, for

$$
\begin{aligned}
\left(1-t^{-1}\right) c_{i j} & =-\left(1-t^{-1}\right)\left(B S^{*} A^{*-1}\right)_{i j} \\
& =-\left(1-t^{-1}\right) \sum_{k=1}^{n} b_{i k}\left(S^{*} A^{*-1}\right)_{k j} \\
& \equiv-\sum_{k=1}^{n} b_{i k}\left\langle\psi \tilde{y}_{m+k}^{*}, \phi \tilde{Y}_{j}\right\rangle \\
& \equiv-\left\langle\psi \sum_{k=1}^{n} b_{i k} \tilde{y}_{m+k}^{*}, \phi \tilde{Y}_{j}\right\rangle
\end{aligned}
$$

which is zero as an element of $\Lambda_{S} / \Lambda$ because the left-hand element in the pairing is zero in $H_{q+1}$. Since $(1-t)$ does not divide det $A$ (or because $(1-t): H_{q} \rightarrow H_{q}$ is an isomorphism), $C$ is a matrix over $\Lambda$.

Now we begin the construction of the requisite $2 q$-knot $k$. Take a presentation with a single 0 -handle, $m(q-1)$-handles $h_{i}^{q-1}(1 \leqslant i \leqslant m)$, and $n$ unknotted $q$-handles $h_{m+i}^{q}(1 \leqslant i \leqslant n)$. Choose bases for $H_{q}(\tilde{Q}, \partial \tilde{Q})$, etc., as in $\S 6$, and embed further $m q$-handles $h_{i}^{q}(1 \leqslant i \leqslant m)$ so that the core of $h_{i}^{q}$ represents $\left[\tilde{v}_{i}\right]=$ $\sum_{j=1}^{m} a_{i j}\left[\tilde{y}_{j}\right]+\sum_{j=1}^{n} s_{i j}\left[\tilde{y}_{m+j}\right]$.

\section{LEMMA 10.1. There is a presentation}

$$
\begin{aligned}
\left\langle\tilde{Y}_{1}, \ldots, \tilde{Y}_{m}, \tilde{y}_{m+1}^{*}, \ldots, \tilde{y}_{m+1}^{*}: 2 \tilde{Y}_{1}, \ldots 2 \tilde{Y}_{m},\right. & \sum_{j=1}^{m} a_{i j} \tilde{Y}_{j}(1 \leqslant i \leqslant m), \\
& \left.-t^{-1} \sum_{j=1}^{m} d_{i j} \tilde{Y}_{j}+\sum_{j=1}^{n} b_{i j} \tilde{y}_{m+j}^{*}(1 \leqslant i \leqslant n)\right\rangle
\end{aligned}
$$

of $\pi_{q+1}$, for some $d_{i j} \in \Lambda$.

Proof. Consider the exact sequence

$$
0 \rightarrow 2 H_{q} \hookrightarrow H_{q} \stackrel{\omega}{\rightarrow} \pi_{q+1} \stackrel{h}{\rightarrow} H_{q+1} \rightarrow 0
$$

which occurs as part of the augmented $F$-form, and define a homomorphism of $\Lambda$-modules

$$
\kappa:\left\langle\tilde{Y}_{1}, \ldots, \tilde{Y}_{m}, \tilde{y}_{m+1}^{*}, \tilde{y}_{m+n}^{*}:\right\rangle \rightarrow \pi_{q+1}
$$

as follows. For $1 \leqslant i \leqslant n$, let $\kappa \tilde{y}_{m+i}^{*}$ be some element of $\pi_{q+1}$ such that $h \kappa \tilde{y}_{m+i}^{*}=$ $\psi \tilde{y}_{m+i}^{*}$ : some such choice is possible because $h$ is an epimorphism. For $1 \leqslant i \leqslant m$, set $\kappa \tilde{Y}_{i}=\omega \phi \tilde{Y}_{i}$. Since $\kappa$ is defined on a free $\Lambda$-module, this extends to a homomorphism. If $x \in \pi_{q+1}$, there is an element $y$ such that $h \kappa y=h x$, since $\psi$ is an epimorphism. 
Thus $x-\kappa y \in \operatorname{ker} h=\operatorname{Im} \omega=\operatorname{Im} \omega \phi$. Hence there is an element $z$ such that $x-\kappa y=\omega \phi z=\kappa z$, and so $x=\kappa(y+z)$, and $\kappa$ is an epimorphism.

It remains to identify the kernel of $\kappa$. Note that

$$
\kappa\left(2 \tilde{Y}_{i}\right)=\omega \phi\left(2 \tilde{Y}_{i}\right)=\omega 2 \phi \tilde{Y}_{i}=0,
$$

and

$$
\kappa\left(\sum_{j=1}^{m} a_{i j} \tilde{Y}_{j}\right)=\omega \phi\left(\sum_{j=1}^{m} a_{i j} \tilde{Y}_{j}\right)=0
$$

Also

$$
h \kappa\left(\sum_{j=1}^{n} b_{i j} \tilde{y}_{m+j}^{*}\right)=\psi\left(\sum_{j=1}^{n} b_{i j} \tilde{y}_{m+j}^{*}\right)=0,
$$

and so there exist $d_{i j}$ such that $\kappa\left(\sum_{j=1}^{n} b_{i j} \tilde{y}_{m+j}^{*}\right)=-t^{-1} \kappa\left(\sum_{j=1}^{m} d_{i j} \tilde{Y}_{j}\right)$. This uses the fact that $\kappa \mid\left\langle\tilde{Y}_{1}, \ldots, \tilde{Y}_{m}\right.$ : $\rangle$ maps onto $\operatorname{Im} \omega=\operatorname{ker} h$. Thus ker $\kappa$ contains all the elements listed as relations in the statement of the lemma: we must now show that these elements span the kernel.

Suppose that $y \in \operatorname{ker} \kappa$. Then $h \kappa y=0$; but $h \kappa$ is defined by $h \kappa \tilde{Y}_{i}=0(1 \leqslant i \leqslant m)$ and $h \kappa \tilde{y}_{m+i}^{*}=\psi \tilde{y}_{m+i}^{*}(1 \leqslant i \leqslant n)$, so there exist $\lambda_{k}$ such that

$$
y=\sum_{k=1}^{n} \lambda_{m+k} \sum_{j=1}^{n} b_{k j} y_{m+j}^{*}+\sum_{k=1}^{m} \lambda_{k} Y_{k} .
$$

Thus

$$
\begin{aligned}
y-\sum_{k=1}^{n} \lambda_{m+k}\left(-t^{-1} \sum_{j=1}^{m} d_{k j} \tilde{Y}_{j}+\sum_{j=1}^{n} b_{k j} \tilde{y}_{m+j}^{*}\right) & \\
= & \sum_{k=1}^{m}\left(\lambda_{k}+t^{-1} \sum_{j=1}^{n} \lambda_{m+j} d_{j k}\right) \tilde{Y}_{k}=\sum_{k=1}^{m} \beta_{k} \tilde{Y}_{k}, \text { say. }
\end{aligned}
$$

Applying $\kappa$ to both sides of this equation, we obtain $\kappa\left(\sum_{k=1}^{m} \beta_{k} \tilde{Y}_{k}\right)=0$, and so $\omega \phi\left(\sum_{k=1}^{m} \beta_{k} \tilde{Y}_{k}\right)=0$. But ker $\omega \phi$ is spanned by $2 \tilde{Y}_{1}, \ldots, 2 \tilde{Y}_{m}, \sum_{j=1}^{m} a_{i j} \tilde{Y}_{j}(1 \leqslant i \leqslant m)$, and so the result is established.

Define the matrix $U$ over $\Gamma_{0}$ by $u_{j k}=\left\{\kappa \tilde{y}_{m+j}, \kappa \tilde{y}_{m+k}^{*}\right\}$; of course some choice is involved here because the pairing $\{$,$\} takes values in \Gamma_{0} / \Gamma$. Define $E$ by the equation $\left(1-t^{-1}\right) E=B U-t^{-1}(1-t) D A^{-1} S$; I claim that $E$ has entries in $\Gamma$. For

$$
\begin{aligned}
\left(1-t^{-1}\right) e_{i k} & =\sum_{j=1}^{n} b_{i j} u_{j k}-t^{-1}(1-t) \sum_{j=1}^{m} d_{i j}\left(A^{-1} S\right)_{j k} \\
& \equiv\left\{\kappa\left(\sum_{j=1}^{n} b_{i j} \tilde{y}_{m+j}^{*}\right), \kappa \tilde{y}_{m+k}^{*}\right\}-t^{-1} \sum_{j=1}^{m} d_{i j} \theta\left\langle\phi \tilde{Y}_{j}, \psi \tilde{y}_{m+k}^{*}\right\rangle \\
& \equiv\left\{\kappa\left(\sum_{j=1}^{n} b_{i j} \tilde{y}_{m+j}^{*}\right), \kappa \tilde{y}_{m+k}^{*}\right\}-t^{-1} \sum_{j=1}^{m} d_{i j}\left\{\kappa \tilde{Y}_{j}, \kappa \tilde{y}_{m+k}^{*}\right\} \\
& \equiv\left\{\kappa\left(\sum_{j=1}^{n} b_{i j} \tilde{y}_{m+j}^{*}-t^{-1} \sum_{j=1}^{m} d_{i j} \tilde{Y}_{j}\right), \kappa \tilde{y}_{m+k}^{*}\right\}
\end{aligned}
$$


which is zero in $\Gamma_{0} / \Gamma$ because the left-hand element in the pairing is zero, and hence $\left(1-t^{-1}\right) E$ has entries in $\Gamma$. But $(1-t): \pi_{q+1} \rightarrow \pi_{q+1}$ is an isomorphism (by the five lemma), and so $E$ has entries in $\Gamma$.

LEMMA 10.2. There exist matrices $M, N, P, Q$ over $\Lambda$ such that

$$
\left(\begin{array}{ll}
M & N \\
C & B
\end{array}\right)\left(\begin{array}{ll}
A^{*} & P^{*} \\
S^{*} & Q^{*}
\end{array}\right)=\left(\begin{array}{ll}
I_{m} & 0 \\
0 & I_{n}
\end{array}\right)
$$

Proof. Note that

$$
\left(\begin{array}{cc}
M & N \\
C & B
\end{array}\right)\left(\begin{array}{ll}
A^{*} & P^{*} \\
S^{*} & Q^{*}
\end{array}\right)=\left(\begin{array}{cc}
M A^{*}+N S^{*} & M P^{*}+N Q^{*} \\
C A^{*}+B S^{*} & C P^{*}+B Q^{*}
\end{array}\right)
$$

and as $C A^{*}+B S^{*}=0$, we already have one of the required identities.

From the work of Blanchfield [B, p. 351], there is a presentation $\left\langle\tilde{v}_{1}^{*}, \ldots, \tilde{v}_{m}^{*}\right.$ : $\left.\sum_{j=1}^{m} a_{i j}^{*} \tilde{v}_{j}^{*}, 1 \leqslant i \leqslant m\right\rangle$ of $H_{q}^{*}$, with quotient map $\psi^{*}:\left\langle\tilde{v}_{1}^{*}, \ldots, \tilde{v}_{m}^{*}:\right\rangle \rightarrow H_{q}^{*}$ such that

$$
\left\langle\psi^{*} \tilde{v}_{i}^{*}, \phi \tilde{Y}_{j}\right\rangle \equiv\left(1-t^{-1}\right)\left(A^{*-1}\right)_{i j}(\bmod \Lambda)
$$

using the fact that $(1-t): H_{q} \rightarrow H_{q}$ is an isomorphism.

Since the pairing $\langle$,$\rangle is nonsingular, we may identify H_{q+1}$ and $H_{q}^{*}$; thus there exists a matrix $N$ over $\Lambda$ such that

$$
\psi^{*} \tilde{v}_{i}^{*}=\sum_{j=1}^{n} n_{i j} \tilde{y}_{m+j}^{*}, \quad 1 \leqslant i \leqslant m .
$$

Substituting for $\psi^{*} \tilde{v}_{i}^{*}$ gives

$$
\begin{aligned}
\left(1-t^{-1}\right)\left(A^{*-1}\right)_{i k} & \equiv\left\langle\psi^{*} \tilde{v}_{i}^{*}, \phi \tilde{Y}_{k}\right\rangle \\
& \equiv\left\langle\sum_{j=1}^{n} n_{i j} \psi \tilde{y}_{m+j}^{*}, \phi \tilde{y}_{k}\right\rangle \\
& \equiv \sum_{j=1}^{n} n_{i j}\left(1-t^{-1}\right)\left(S^{*} A^{*-1}\right)_{j k}
\end{aligned}
$$

Thus there exists a matrix $M$ over $\Lambda$ such that

$$
\left(1-t^{-1}\right) A^{*-1}=\left(1-t^{-1}\right) N S^{*} A^{*-1}+\left(1-t^{-1}\right) M
$$

(this uses the fact that $(1-t)$ does not divide $\operatorname{det} A$ ) and hence

$$
I=N S^{*}+M A^{*}
$$

which is another of the identities we require.

Appealing again to [B, p. 351], there is a presentation

$$
\left\langle\tilde{V}_{m+1}, \ldots, \tilde{V}_{m+n}: \sum_{j=1}^{n} b_{i j}^{*} \tilde{V}_{m+j}, 1 \leqslant i \leqslant n\right\rangle
$$

of $H_{q}$ such that

$$
\left\langle\psi y_{m+i}^{*}, \phi^{*} V_{m+k}\right\rangle \equiv\left(1-t^{-1}\right)\left(B^{-1}\right)_{i k} \quad(\bmod \Lambda) .
$$


From the identification of $H_{q+1}$ and $H_{q}^{*}$, there exists $P$ over $\Lambda$ such that $\phi^{*} \tilde{V}_{m+k}=-\sum_{j=1}^{m} \phi p_{k j} \tilde{Y}_{j}$. Then

$$
\begin{aligned}
\left(1-t^{-1}\right)\left(B^{-1}\right)_{i k} & \equiv\left\langle\psi \tilde{y}_{m+i}^{*}, \phi^{*} \tilde{V}_{m+k}\right\rangle \equiv\left\langle\psi \tilde{y}_{m+i}^{*},-\sum_{j=1}^{m} \phi p_{k j} \tilde{Y}_{j}\right\rangle \\
& \equiv-\sum_{j=1}^{m} p_{k j}\left\langle\psi \tilde{y}_{m+i}^{*}, \phi \tilde{Y}_{j}\right\rangle \equiv-\sum_{j=1}^{m} p_{k j}\left(1-t^{-1}\right)\left(S^{*} A^{*-1}\right)_{i j} \\
& \equiv-\left(1-t^{-1}\right)\left(S^{*} A^{*-1} P^{*}\right)_{i k}
\end{aligned}
$$

and so there exists a matrix $Q$ over $\Lambda$ such that

$$
\begin{aligned}
B^{-1} & =-S^{*} A^{*-1} P^{*}+Q^{*}, \\
I & =-B S^{*} A^{*-1} P^{*}+B Q^{*}, \\
I & =C P^{*}+B Q^{*},
\end{aligned}
$$

which is another of the identities needed.

Note that $P$ is not unique; since $\phi\left(\sum_{j=1}^{m} a_{i j} \tilde{Y}_{j}\right)=0$, we could replace $p_{k j}$ by $p_{k j}+\sum_{j=1}^{m} \pi_{k i} a_{i j}$. Thus $P$ can be replaced by $P+\Pi A$. It follows from the equation

$$
B^{-1}=-S^{*} A^{*-1}\left(P^{*}+A^{*} \Pi^{*}\right)+Q^{*}+S^{*} \Pi^{*}
$$

that $Q^{*}$ must then be replaced by $Q^{*}+S^{*} \Pi^{*}$.

We then have

$$
C\left(P^{*}+A^{*} \Pi^{*}\right)+B\left(Q^{*}+S^{*} \Pi^{*}\right)=C P^{*}+B Q^{*}+\left(C A^{*}+B S^{*}\right) \Pi^{*}=I,
$$

and

$$
\begin{aligned}
M\left(P^{*}+A^{*} \Pi^{*}\right)+N\left(Q^{*}+S^{*} \Pi^{*}\right) & =M P^{*}+N Q^{*}+\left(M A^{*}+N S^{*}\right) \Pi^{*} \\
& =M P^{*}+N Q^{*}+\Pi^{*} .
\end{aligned}
$$

Choosing $\Pi$ so that $M P^{*}+N Q^{*}+\Pi^{*}=0$ completes the proof.

LEMMA 10.3. $D C^{*}+t C D^{*}=B T B^{*}$ for some matrix $T$ over $\Gamma$, such that $T^{*}=t T$.

Proof. Recall that

$$
\left(1-t^{-1}\right) c_{i k} \equiv-\left\langle\psi \sum_{j=1}^{n} b_{i j} \tilde{y}_{m+j}^{*}, \phi \tilde{Y}_{k}\right\rangle \quad(\bmod \Lambda)
$$

This implies that

$$
\begin{gathered}
\left(1-t^{-1}\right) c_{i k} \equiv-\left\{\kappa \sum_{j=1}^{n} b_{i j} \tilde{y}_{m+j}^{*}, \kappa \tilde{Y}_{k}\right\} \quad(\bmod \Gamma), \\
\left(1-t^{-1}\right)\left(B^{-1} C\right)_{i k} \equiv-\left\{\kappa \tilde{y}_{m+i}^{*}, \kappa \tilde{Y}_{k}\right\}, \\
\left(1-t^{-1}\right)\left(B^{-1} C D^{*}\right)_{i k} \equiv-\left\{\kappa \tilde{y}_{m+i}^{*}, \kappa \sum_{j=1}^{m} d_{k j} \tilde{Y}_{j}\right\} .
\end{gathered}
$$


Since $\kappa\left(-t^{-1} \sum_{j=1}^{m} d_{k j} \tilde{Y}_{j}+\sum_{j=1}^{n} b_{k j} \tilde{y}_{m+j}^{*}\right)=0$, this gives

$$
\begin{gathered}
\left(1-t^{-1}\right)\left(B^{-1} C D^{*}\right)_{i k} \equiv\left\{\kappa \tilde{y}_{m+i}^{*}, \kappa t \sum_{j=1}^{n} b_{k j} \tilde{y}_{m+j}^{*}\right\}, \\
(t-1)\left(B^{-1} C D^{*} B^{*-1}\right)_{i k} \equiv\left\{\kappa \tilde{y}_{m+i}^{*}, \kappa \tilde{y}_{m+k}^{*}\right\}
\end{gathered}
$$

and so $\left(t^{-1}-1\right)\left(B^{-1} D C^{*} B^{*-1}\right)_{i k} \equiv \overline{\left\{\kappa \tilde{y}_{m+k}^{*}, \kappa \tilde{y}_{m+i}^{*}\right\}}$.

But $\{\kappa x, \kappa y\}=\overline{\{\kappa y, \kappa x\}}$, and so

$$
\begin{gathered}
\left(t^{-1}-1\right)\left(B^{-1} D C^{*} B^{*-1}-(t-1) B^{-1} C D^{*} B^{*-1} \equiv 0 \quad(\bmod \Gamma),\right. \\
\left(1-t^{-1}\right) B^{-1}\left(D C^{*}+t C D^{*}\right) B^{*-1} \equiv 0 \quad(\bmod \Gamma) .
\end{gathered}
$$

Since $(1-t)$ does not divide $\operatorname{det} B$, this implies that

$$
B^{-1}\left(D C^{*}+t C D^{*}\right) B^{*-1}=T
$$

for some matrix $T$ over $\Gamma$, as desired. Finally, $B T^{*} B^{*}=\left(B T B^{*}\right)^{*}=C D^{*}+t^{-1} D C^{*}$ $=t B T B^{*}$, and since $B$ is nonsingular, this gives $T^{*}=t T$.

LEMma 10.4. $E$ can be chosen so that $D C^{*}+E B^{*}-t\left(C D^{*}+B E^{*}\right)=0$.

Proof. Substituting $\left(1-t^{-1}\right) E=B U+\left(1-t^{-1}\right) D A^{-1} S$ gives

$$
\begin{aligned}
\left(1-t^{-1}\right)\left[D C^{*}\right. & \left.+E B^{*}-t\left(C D^{*}+B E^{*}\right)\right] \\
= & \left(1-t^{-1}\right)\left(D C^{*}-t C D^{*}\right)+B U B^{*}+\left(1-t^{-1}\right) D A^{-1} S B^{*} \\
& +B U^{*} B^{*}+(1-t) B S^{*} A^{*-1} D^{*} \\
= & \left(1-t^{-1}\right)\left(D C^{*}-t C D^{*}\right) \\
& +B\left(U+U^{*}\right) B^{*}+\left(1-t^{-1}\right) D C^{*}+(1-t) C D^{*} \\
= & B\left(U+U^{*}\right) B^{*}
\end{aligned}
$$

since we are working mod2. Recall that from the proof of Lemma 10.3, $U \equiv$ $(t-1) B^{-1} C D^{*} B^{-1}(\bmod \Gamma)$. Thus we can set

$$
U=(t-1) B^{-1} C D^{*} B^{*-1}+\left(t^{-1}-1\right) K,
$$

where $K$ is a matrix over $\Gamma$ to be chosen later. Thus

$$
\begin{aligned}
B\left(U+U^{*}\right) B^{*} & =(t-1) C D^{*}+\left(t^{-1}-1\right) D C^{*}+\left(t^{-1}-1\right) B\left(K+t K^{*}\right) B^{*} \\
& =\left(t^{-1}-1\right)\left[t C D^{*}+D C^{*}+B\left(K+t K^{*}\right) B^{*}\right] \\
& =\left(t^{-1}-1\right) B\left(T+K+t K^{*}\right) B^{*}
\end{aligned}
$$

by Lemma 10.3. The argument of [K5, pp. 157-158] shows that $K$ may be chosen so that $T+K+t K^{*}=0$, and this completes the proof.

Now we construct the required $2 q$-knot $k$. Take a presentation with a single 0 -handle, $m(q-1)$-handles $h_{i}^{q-1}(1 \leqslant i \leqslant m)$, and $n$ unknotted $q$-handles $h_{m+i}^{q}$ $(1 \leqslant i \leqslant n)$. This gives an exterior $Q$ as in $\S 6$, and we choose bases for $H_{q}(\tilde{Q}, \partial \tilde{Q})$, etc., as in that section. Define $\left[\tilde{v}_{i}\right] \in H_{q}(\tilde{Q}, \partial \tilde{Q})$ by

$$
\left[\tilde{v}_{i}\right]=\sum_{j=1}^{m} a_{i j}\left[\tilde{y}_{j}\right]+\sum_{j=1}^{n} s_{i j}\left[\tilde{y}_{m+j}\right], \quad 1 \leqslant i \leqslant m .
$$


By Lemma 10.2, the matrix $\left({ }_{P}^{A} S\right)$ is invertible over $\Lambda$, and hence the $\left[\tilde{v}_{i}\right]$, $1 \leqslant i \leqslant m$, form part of a basis for the free $\Lambda$-module $H_{q}(\tilde{Q}, \partial \tilde{Q})$. By the Hurewicz theorem, $\pi_{q}(\tilde{Q}, \partial \tilde{Q}) \cong H_{q}(\tilde{Q}, \partial \tilde{Q})$, and each $\left[\tilde{v}_{i}\right]$ may be represented by a map $f_{i}$ : $\left(B^{q}, \partial B^{q}\right) \rightarrow(Q, \partial Q)$. Recalling that $\partial Q=M \times S^{1}$, we see that $\pi_{q-1}(\partial Q) \cong$ $\pi_{q-1}(M) \oplus \pi_{q-1}\left(S^{1}\right)=\pi_{q-1}(M)$, and so $f_{i}$ may be homotoped until $f_{i}\left(\partial B^{q}\right) \subset M_{1}$ for some $M_{1}=M \times * \subset M \times S^{1}$. By general position, homotop $f_{i}$ until $f_{i}: \partial B^{q} \rightarrow M_{1}$ are disjoint embeddings, and then by general position homotop $\cup_{i=1}^{m} f_{i}$ rel $\partial$ until it is an embedding. We can then take $f_{i}\left(B^{q}\right)$ as the core of a $q$-handle $\bar{h}_{i}^{q}$ with its attaching tube in $M_{1}$, and hence obtain a $q$-handle $h_{i}^{q}$ of $k$.

Next define $\left(\tilde{V}_{m+i}^{*}\right) \in \pi_{q+1}(\tilde{Q}, \partial \tilde{Q})$ by

$$
\left(\tilde{V}_{m+i}^{*}\right)=\sum_{j=1}^{m} c_{i j}\left(\tilde{Y}_{j}^{*}\right)+\sum_{j=1}^{n} b_{i j}\left(\tilde{Y}_{m+j}^{*}\right)+\sum_{j=1}^{m} d_{i j}\left(\tilde{y}_{j}\right) \circ \xi+\sum_{j=1}^{n} e_{i j}\left(\tilde{y}_{m+j}\right) \circ \xi,
$$

and represent $\left(\tilde{V}_{m+i}^{*}\right)$ by a map $g_{m+i}:\left(B^{q+1}, \partial B^{q+1}\right) \rightarrow(Q, \partial Q)$. As above, we can homotop $g_{m+i}$ until $g_{m+i}\left(\partial B^{q+1}\right) \subset M_{1}$. The algebraic intersection of $g_{m+i}\left(\partial B^{q+1}\right)$ with $f_{j}\left(\partial B^{q}\right)$ is easily seen to be $S_{M_{1}}\left(\left[\partial \tilde{V}_{m+i}^{*}\right],\left[\partial \tilde{v}_{j}\right]\right)=S_{\tilde{Q}}\left(\left[\tilde{V}_{m+i}^{*}\right],\left[\tilde{v}_{j}\right]\right)_{t=1}=$ $\left(C(1) A^{*}(1)+B(1) S^{*}(1)\right)_{i j}=0$ by Lemma 6.14. Here $C(1)$ denotes $C$ with $t=1$. By the Whitney trick, we can homotop the $g_{m+i}$ until $\cup_{i=1}^{n} g_{m+i}\left(\partial B^{q+1}\right) \cap$ $\cup_{i=1}^{m} f_{i}\left(\partial B^{q}\right)=\varnothing$. In a similar spirit,

$T_{M_{1}}\left(\left(\partial \tilde{V}_{m+i}^{*}\right),\left(\partial \tilde{V}_{m+j}^{*}\right)\right)=\left[D(1) C^{*}(1)+E(1) B^{*}(1)+C(1) D^{*}(1)+B(1) E^{*}(1)\right]_{i j}$.

(Compare the proof of Lemma 6.18.) Since the matrix on the right is zero, the results of [K1] show that $\cup_{i=1}^{n} g_{m+i}$ may be homotoped to be an embedding on the boundary. As in Lemma 6.19, $\cup_{i=1}^{n} g_{m+i}$ can be homotoped rel $\partial$ to an embedding, and then $g_{m+i}\left(B^{q+1}\right)$ yields a $(q+1)$-handle $h_{m+i}^{q+1}$ of $k$ corresponding to $\left(\tilde{V}_{m+i}^{*}\right)$.

By the homology properties of $\left[\tilde{v}_{i}\right]$ and $\left[\tilde{V}_{m+j}^{*}\right]$ (compare $\left.\S 6\right)$, after adding in all the handles of $k$ constructed so far, we obtain an unknotted $S^{2 q-1}$, which may be capped off to complete the construction of $k$.

11. The Seifert surface. Let $k$ be a simple $Z$-torsion-free $2 q$-knot, $q \geqslant 4$. By Lemma II.11 of [Ke], there exists a Seifert surface $V$ of $k$ which is $(q-1)$-connected and has $H_{q}(V)$ torsion-free.

Choose a basis $\alpha_{l}^{q}, \ldots, \alpha_{r}^{q}$ of $H_{q}(V)$ : this gives rise to a dual basis $\alpha_{1}^{q+1}, \ldots, \alpha_{r}^{q+1}$ of $H_{q+1}(V)$ by the intersection pairing $I: H_{q+1}(V) \times H_{q}(V) \rightarrow \mathrm{Z}$, by Poincaré duality. There is also a basis $\beta_{1}^{q+1}, \ldots, \beta_{r}^{q+1}$ of $H_{q+1}\left(S^{2 q+2}-V\right)$, dual to $\alpha_{1}^{q}, \ldots, \alpha_{r}^{q}$ under the linking pairing $L: H_{q}(V) \times H_{q+1}\left(S^{2 q+2}-V\right) \rightarrow \mathbf{Z}$ by Alexander duality. Thus

$$
I\left(\alpha_{i}^{q+1}, \alpha_{j}^{q}\right)=\delta_{i j}=L\left(\alpha_{j}^{q}, \beta_{i}^{q+1}\right) .
$$

Similarly there is a basis $\beta_{1}^{q}, \ldots, \beta_{r}^{q}$ of $H_{q}\left(S^{2 q+2}-V\right)$ such that $L\left(\alpha_{i}^{q+1}, \beta_{j}^{q}\right)=\delta_{i j}$.

Let $i_{ \pm}: H_{*}(V) \rightarrow H_{*}\left(S^{2 q+2}-V\right)$ be induced by translating cycles off $V$ in the positive/negative direction, and define the integer matrices $A, B$ by

$$
i_{+} \alpha_{i}^{q}=\sum_{j=1}^{r} a_{i j} \beta_{j}^{q}, \quad i_{-} \alpha_{i}^{q}=\sum_{j=1}^{r} b_{i j} \beta_{j}^{q} .
$$

Then $H_{q}(\tilde{K})$ is presented as a $\Lambda$-module by the matrix $t A-B$. As in [L2], the corresponding matrix for $H_{q+1}(\tilde{K})$ is $(-1)^{q(q+1)} t\left(t^{-1} A-B\right)^{\prime}=A^{\prime}-t B^{\prime}$. Let $\phi^{s}$ : $\left\langle\beta_{1}^{s}, \ldots, \beta_{r}^{s}:\right\rangle \rightarrow H_{s}(\tilde{K})$ be the quotient map. Then arguing as in [K5], the Blanchfield 
pairing is given by

$$
\left\langle\phi^{q+1} \beta_{i}^{q+1}, \phi^{q} \beta_{j}^{q}\right\rangle \equiv(1-t)\left(A^{\prime}-t B^{\prime}\right)_{i j}^{-1}(\bmod \Lambda) .
$$

Just as $H_{q}(\tilde{K})$ is presented by $t A-B$, so $\pi_{q+1}(\tilde{K})$ has a presentation as a $\Lambda$-module:

$$
\begin{aligned}
\left\langle\beta_{1}^{q}, \ldots, \beta_{r}^{q}, \beta_{1}^{q+1}, \ldots, \beta_{r}^{q+1}:\right. & 2 \beta_{1}^{q}, \ldots, 2 \beta_{r}^{q}, \sum_{j=1}^{r}(t A-B)_{i j} \beta_{j}^{q}(1 \leqslant i \leqslant r), \\
& \left.\sum_{j=1}^{r}(t C-D)_{i j} \beta_{j}^{q}+\sum_{j=1}^{r}\left(A^{\prime}-t B^{\prime}\right)_{i j} \beta_{j}^{q+1} \quad(1 \leqslant i \leqslant r)\right\rangle
\end{aligned}
$$

where the matrices $C, D$ are defined as follows.

$$
\begin{gathered}
i_{+}\left(\alpha_{i}^{q+1}\right)=\sum_{j=1}^{r}-b_{j i}\left(\beta_{j}^{q+1}\right)+\sum_{j=1}^{r} c_{i j}\left(\beta_{j}^{q}\right) \circ \zeta, \\
i_{-}\left(\alpha_{i}^{q+1}\right)=\sum_{j=1}^{r}-a_{j i}\left(\beta_{j}^{q+1}\right)+\sum_{j=1}^{r} d_{i j}\left(\beta_{j}^{q}\right) \circ \zeta
\end{gathered}
$$

where as before ( ) denotes a homotopy class and $\zeta$ represents the nonzero element of $\pi_{q+1}\left(S^{q}\right)$.

Recalling that $\Pi_{q+1}(\tilde{K})=\pi_{q+1}(\tilde{K}) / 2 \pi_{q+1}(\tilde{K})$, we obtain the following presentation for $\Pi_{q+1}(\tilde{K})$ as a $\Gamma$-module:

$$
\begin{aligned}
\left\langle\beta_{1}^{q}, \ldots, \beta_{r}^{q}, \beta_{1}^{q+1}, \ldots, \beta_{r}^{q+1}:\right. & \sum_{j=1}^{r}(t A-B)_{i j} \beta_{j}^{q}(1 \leqslant i \leqslant r), \\
& \left.\sum_{j=1}^{r}(t C-D)_{i j} \beta_{j}^{q}+\sum_{j=1}^{r}\left(A^{\prime}-t B^{\prime}\right)_{i j} \beta_{j}^{q+1}(1 \leqslant i \leqslant r)\right\rangle .
\end{aligned}
$$

Let $\eta:\left\langle\beta_{1}^{q}, \ldots, \beta_{r}^{q}, \beta_{1}^{q+1}, \ldots, \beta_{r}^{q+1}:\right\rangle \rightarrow \Pi_{q+1}(\tilde{K})$ denote the quotient map, and $\mathcal{L}$ : $\pi_{q+1}(V) \times \pi_{q+1}\left(S^{2 q+2}-V\right) \rightarrow \mathbf{Z}_{2}$ the homotopy linking. We can alter $\left(\beta_{i}^{q+1}\right)$ by elements $\left(\beta_{k}^{q}\right) \circ \zeta$ until $\varrho\left(\left(\alpha_{i}^{q+1}\right),\left(\beta_{j}^{q+1}\right)\right)=0$ for all $i, j$. Then

$$
c_{i k}=\mathcal{L}\left(\left(\alpha_{k}^{q+1}\right), i_{+}\left(\alpha_{i}^{q+1}\right)\right)=\mathcal{L}\left(i_{-}\left(\alpha_{k}^{q+1}\right), \alpha_{i}^{q+1}\right)=d_{k i}
$$

and so $D=C^{\prime}$. Then we can compute the pairing [ , ]:

$$
\begin{gathered}
{\left[\eta \beta_{i}^{q+1}, \eta\left(\sum_{j=1}^{r}\left(t C-C^{\prime}\right)_{k j} \beta_{j}^{q}+\sum_{j=1}^{r}\left(A^{\prime}-t B^{\prime}\right)_{k j} \beta_{j}^{q+1}\right)\right] \equiv 0} \\
\sum_{j=1}^{r}\left(t^{-1} C-C^{\prime}\right)_{k j}\left[\eta \beta_{i}^{q+1}, \eta \beta_{j}^{q}\right]+\sum_{j=1}^{r}\left(A^{\prime}-t^{-1} B^{\prime}\right)_{k j}\left[\eta \beta_{i}^{q+1}, \eta \beta_{j}^{q+1}\right] \equiv 0 \\
\sum_{j=1}^{r}\left(A^{\prime}-t^{-1} B^{\prime}\right)_{k j}\left[\eta \beta_{i}^{q+1}, \eta \beta_{j}^{q+1}\right] \equiv \sum_{j=1}^{r}\left(t^{-1} C-C^{\prime}\right)_{k j}(1-t)\left(A^{\prime}-t B^{\prime}\right)_{i j}^{-1}, \\
{\left[\eta \beta_{i}^{q+1}, \eta \beta_{l}^{q+1}\right] \equiv(1-t) \sum_{j=1}^{r} \sum_{k=1}^{r}\left(t^{-1} C-C^{\prime}\right)_{k j}\left(A^{\prime}-t B^{\prime}\right)_{i j}^{-1}\left(A^{\prime}-t^{-1} B^{\prime}\right)_{l k}^{-1}} \\
\equiv(1-t)\left[\left(A^{\prime}-t B^{\prime}\right)^{-1}\left(t^{-1} C^{\prime}-C\right)\left(A-t^{-1} B\right)^{-1}\right]_{i l} .
\end{gathered}
$$


At this point we wish to use the results of [K6]. But first we must point out that Theorems 3.1, 3.2 and 13.1 of that paper are only valid for $q \geqslant 4$. There is an assumption in the proof of Lemma 6.1 of [K6] that certain normal bundles are trivial, which I am unable to justify. However, the lemma can be proved (for $q \geqslant 4$ ) by appealing to [Ko, Lemma 3] or to the techniques of [K1].

As indicated in $[K 6, \S 13]$, the matrices $A, B$ and $C$ can be obtained from a $(-1)^{q}$-form associated with the knot $k$, where $q \geqslant 4$, by a choice of bases. Thus, via the geometrical theorems of the present paper and [K6], for $q \geqslant 6$, we obtain

THEOREM 11.1. Two Z-torsion-free $(-1)^{q}$-forms are T-equivalent if and only if they present isometric F-forms.

COROLlaRY 11.2. Theorem 2.1 is true for $q \geqslant 4$.

COROLlaRY 11.3. Theorem 2.2 is true for $q \geqslant 4$.

COROLlaRY 11.4. Theorem 2.3 is true for $q \geqslant 4$.

12. An example. Some of the invariants studied in this paper are not new; for example $H_{q}(\tilde{K})$ and its associated ideals and polynomials. On the other hand $\Pi_{q+1}(\tilde{K})$ and the associated Hermitian form $[,]_{\tilde{K}}$ are new, although it has arisen in another form as a $\mathbf{Z}_{2}$ homotopy pairing in [K6 and Ko]. So far as I am aware, no example has yet been given to show that this $\mathbf{Z}_{2}$-pairing is necessary, and the reason for this is that it is difficult to find an invariant of the knot which depends on the $\mathbf{Z}_{2}$-pairing. The explanation for this is as follows. Being a $\Gamma$-module, we can apply the analysis of $[\mathbf{M 1}]$ (as modified in $[\mathbf{K} 4])$ to $\left(\Pi_{q+1}(\tilde{K}),[,]_{\tilde{K}}\right)$. That is, $\Pi_{q+1}(\tilde{K})$ can be written as the orthogonal direct sum

$$
\frac{1}{p(t)} M_{p(t)} \frac{1}{q(t)} N_{q(t)}
$$

where $(p(t))=\left(p\left(t^{-1}\right)\right)$ and $M_{p(t)}$ is the $p(t)$-primary component, $(q(t)) \neq\left(q\left(t^{-1}\right)\right)$ and $N_{q(t)}$ is the direct sum of the $q(t)$ and $q\left(t^{-1}\right)$-primary components. Concentrating on the former case, each $M_{p(t)}$ can be written as the orthogonal direct sum $M_{1} \perp M_{2} \perp \cdots \perp M_{r}$, where each $M_{i}$ is the direct sum of modules of the form $\Gamma /\left(p(t)^{i}\right)$. Then $M_{i} / p(t) M_{i}$ is a vector space over the finite field $\Gamma /(p(t))$, and in fact it is a Hermitian vector space by $((x),(y))=\left[p(t)^{i-1} x, y\right]$. But a Hermitian vector space over a finite field is determined by its rank (cf. [M1]), so we obtain no new invariants by this means. Worse still, it is not hard to show that the Hermitian vector spaces so defined determine the original $\Gamma$-module and pairing, so that it looks as though $[,]_{\tilde{K}}$ contributes nothing.

Consider the following examples. Let $H_{q}=\Lambda /\left(1-t+t^{2}\right) \oplus \Lambda /\left(1-3 t+t^{2}\right)$ with generators $a, b$. Then $\mathcal{H}_{q} \equiv \Gamma /\left(1-t+t^{2}\right) \oplus \Gamma /\left(1-t+t^{2}\right)$, and we define $\Pi_{q+1}=\Gamma /\left(1-t+t^{2}\right)^{2} \oplus \Gamma /\left(1-t+t^{2}\right)^{2}$, with generators $\alpha, \beta$. We define Hermitian forms [ , ] and $\langle$,$\rangle on \Pi_{q+1}$ by

$$
\begin{aligned}
& {[\alpha, \alpha]=\frac{1}{\left(1-t+t^{2}\right)^{2}}=[\beta, \beta], \quad[\alpha, \beta]=0,} \\
& \langle\alpha, \alpha\rangle=0=\langle\beta, \beta\rangle, \quad\langle\alpha, \beta\rangle=\frac{1}{\left(1-t+t^{2}\right)^{2}} .
\end{aligned}
$$


Let $p_{q}(a)=\left(1-t+t^{2}\right) \alpha, p_{q}(b)=\left(1-t+t^{2}\right) \beta$, where $\mathcal{H}_{q}$ is identified with $(1-t+t) \Pi_{q+1}$. Then $\mathcal{H}_{q} \hookrightarrow \Pi_{q+1} \rightarrow \Pi_{q+1} / \mathcal{H}_{q}$ defines a s.e.s. $\mathcal{E}$, and setting $\mathcal{H}_{q+1}=\Pi_{q+1} / \mathcal{H}_{q}$, it is clear that [, ] and $\langle$,$\rangle each define a nonsingular Hermitian$ form $\mathcal{H}_{q+1} \times \mathcal{H}_{q} \rightarrow \Gamma_{0} / \Gamma$. Hence we have two $F$-forms, which correspond to two knots $k$ and $l$ say. Although it is not hard to see that $\left(\Pi_{q+1},[],\right)$ is isometric to $\left(\Pi_{q+1},\langle\rangle,\right)$, by changing basis, it is equally clear that the $F$-forms are not isometric, for the form of $H_{q}$ means that the change of basis permitted in $\Pi_{q+1}$ is restricted to one of the form $\alpha \mapsto \varepsilon \alpha+\left(1-t+t^{2}\right) \beta, \beta \mapsto \varepsilon^{\prime} \beta+\left(1-t+t^{2}\right) \alpha$, where $\varepsilon, \varepsilon^{\prime}$ are units of $\Gamma \bmod \left(1-t+t^{2}\right)^{2}$ and this is not sufficient to transform $\left(\Pi_{q+1},[],\right)$ into $\left(\Pi_{q+1},\langle\rangle,\right)$. Thus $k \neq l$.

\section{REFERENCES}

[Ba] H. Bass, Algebraic K-theory, Benjamin, New York, 1968.

[B] R. C. Blanchfield, Intersection theory of manifolds with operators, with applications to knot theory, Ann. of Math. 65 (1957), 340-356.

[F] R. H. Fox, A quick trip through knot theory, Topology of 3 Manifolds and Related Topics (Proc. Univ. of Georgia Inst., 1961), Prentice-Hall, Englewood Cliffs, N. J., 1962, pp. 120-167.

[Fa] M. S. Faber, Isotopy types of knots of codimension two, Trans. Amer. Math. Soc. 261 (1980), 185-209.

[H] S. T. Hu, Homotopy theory, Academic Press, New York, 1959.

[K1] C. Kearton, Obstructions to embedding and isotopy in the metastable range, Math. Ann. 243 (1979), 103-113.

[K2] _ Attempting to classify knot modules and their Hermitian pairings, Knot Theory, Plans-surBex 1977 (Ed. J. C. Hausmann), Lecture Notes in Math., vol. 685, Springer-Verlag, Berlin and New York, 1978, pp. 227-242.

[K3] _ Presentations of $n-k n o t s$, Trans. Amer. Math. Soc. 202 (1975), 123-140.

[K4] _ Signatures of knots and the free differential calculus, Quart. J. Math. Oxford Ser. (2) 30 (1979), 157-182.

[K5] __, Blanchfield duality and simple knots, Trans. Amer. Math. Soc. 202 (1975), 141-160.

[K6] _ An algebraic classification of some even-dimensional knots, Topology 15 (1976), 363-373.

[K-L] C. Kearton and W. B. R. Lickorish, Piecewise linear critical levels and collapsing, Trans. Amer. Math. Soc. 170 (1972), 415-424.

[Ke] M. A. Kervaire, Les noeuds de dimensions supérieures, Bull. Soc. Math. France 93 (1965), 225-271.

[Ko] S. Kojima, A classification of some even dimensional fibred knots, J. Fac. Sci. Univ. Tokyo Sect. IA Math. 24 (1977), 671-683.

[Ko2] _ Classification of simple knots by Levine pairings, Comment. Math. Helv. 54 (1979), 356-367.

[L] J. Levine, Knot modules. I, Trans. Amer. Math. Soc. 229 (1977), 1-50.

[L2] __ Polynomial invariants of knots of codimension two, Ann. of Math. 84 (1966), 537-554.

[L3] _ An algebraic classification of some knots of codimension two, Comment. Math. Helv. 45 (1970), 185-198.

[Ma] S. Mac Lane, Homology, Springer-Verlag, Berlin and New York, 1975.

[M] J. Milnor, Introduction to algebraic K-theory, Ann. of Math. Studies, no. 72, Princeton Univ. Press, Princeton, N. J., 1971.

[N] D. G. Northcott, Homological algebra, Cambridge Univ. Press, New York, 1960.

Department of Mathematics, University of Durham, Durham DH1 3LE, England 